\title{
A reconstruction of Proto-Jê phonology and lexicon ${ }^{1}$
}

\begin{abstract}
In this work, I examine the sound correspondences between Proto-Cerrado (Nikulin 2017) and Proto-Southern Jê (Jolkesky 2010) and offer a phonological reconstruction of Proto-Jê, the proto-language of the most diverse subgrouping within the Macro-Jê language stock. I reconstruct 11 consonants and 19 vowels for this proto-language. I also claim that */CrVC/ was the maximal syllable structure in Proto-Jê with some further restrictions on its complex onsets (only */pr, mr, kr, $\mathrm{pr} /$ were allowed). I reconstruct a shielding allophony pattern to Proto-Jê, according to which nasal onsets would have had post-oralized allophones before oral nuclei. The discussion on Proto-Jê phonology is followed by a sample of Proto-Jê lexicon.
\end{abstract}

Keywords: Jê languages, Macro-Jê languages, language reconstruction, comparative method.

\section{Introduction}

Since the pioneer work of Davis (1966), which remains the only published attempt at a reconstruction of Proto-Jê to the present day, numerous descriptive studies on individual Jê languages have been carried out, contributing to a significant improvement in the state-of-theart of Jê linguistics. Recent comparative work on low-level branches of Jê, such as Southern Jê, Northern Jê, Central Jê, and Cerrado (Wiesemann 1978, Jolkesky 2010, Nikulin \& Salanova forthc., Nikulin 2016, 2017), has enabled the use of intermediate reconstructions in comparative studies on higher levels. In this paper, I offer a revised reconstruction of Proto-Jê, taking into account the research on Jê produced over the last 50 years.

I follow the principles of bottom-up reconstruction, which means that my reconstruction of Proto-Jê is based on the reconstructions of its daughter proto-languages, Proto-Cerrado (Nikulin 2017) and Proto-Southern Jê (Wiesemann 1978, Jolkesky 2010); the former, in turn, is based on the reconstructions of Proto-Northern Jê (Nikulin 2016, cf. also Nikulin \& Salanova forthc.) and Proto-Central Jê (Nikulin 2017). This allows to filter out recent phonetic, morphological, and semantic innovations, which is especially important in light of the conservativeness traditionally attributed to Northern Jê (cf. Ribeiro \& Voort 2010: 560, Carvalho 2016: 64, Pache 2018); it is well known that over-reliance on the data of a given

${ }^{1}$ I gratefully acknowledge the financial support of CAPES (Coordenação de Aperfeiçoamento de Pessoal de Nível Superior) during my doctoral studies. I also thank Andrés Pablo Salanova, Mário André Coelho da Silva, and Matthias Pache for valuable input that helped improve this paper. Furthermore, its text has benefited from the comments of Juho Pystynen, Mikhail A. Zhivlov, and Lev Michael, to whom I am grateful. I warmly thank my Jê-speaking friends, especially Bepo Metyktire and Eneida Xerente, for teaching me bits of their languages. Finally, I thank the audience at Amazónicas VI (Tabatinga), where a much earlier version of this paper was presented on May 27th, 2016.

I use the following abbreviations throughout the paper: PCerr = Proto-Cerrado, PCJ = Proto-Central Jê, PJ = Proto-Jê, PNJ = Proto-Northern Jê, PSJ = Proto-Southern Jê, ABL =ablative, ACT =active, ADESS $=$ adessive, $\mathrm{ADVT}=$ advertence prohibitive,$\quad \mathrm{ALL}=$ allative,$\quad \mathrm{CAUS}=$ causative $, \quad \mathrm{COP}=\mathrm{copula}, \quad \mathrm{DU}=\mathrm{dual}, \quad \mathrm{ERG}=\mathrm{ergative}$, HABIT $=$ habitual, INSTR $=$ instrumental, INTR $=$ intransitive, $\mathrm{LOC}=$ locative, MALEF $=$ malefactive, NEG $=$ negative, $\mathrm{NF}=$ nonfinite, NMLZ.AG = agent nominalization, $\mathrm{PL}=$ plural, $\mathrm{SG}=$ singular, STAT = stative, ATR = advanced tongue root. 
language or language group has repeatedly led historical linguists to wrong reconstructive solutions. ${ }^{2}$ It is important to emphasize that bottom-up reconstruction does not exclude occasional use of external material, which may be invoked in situations when internal data offer conflicting or incomplete evidence.

My working model of the Jê phylogenetic tree (without Xakriabá and Akroá, whose position within Central Jê is uncertain) is presented in Figure $1 .^{3}$

Figure 1. Internal structure of the Jê family

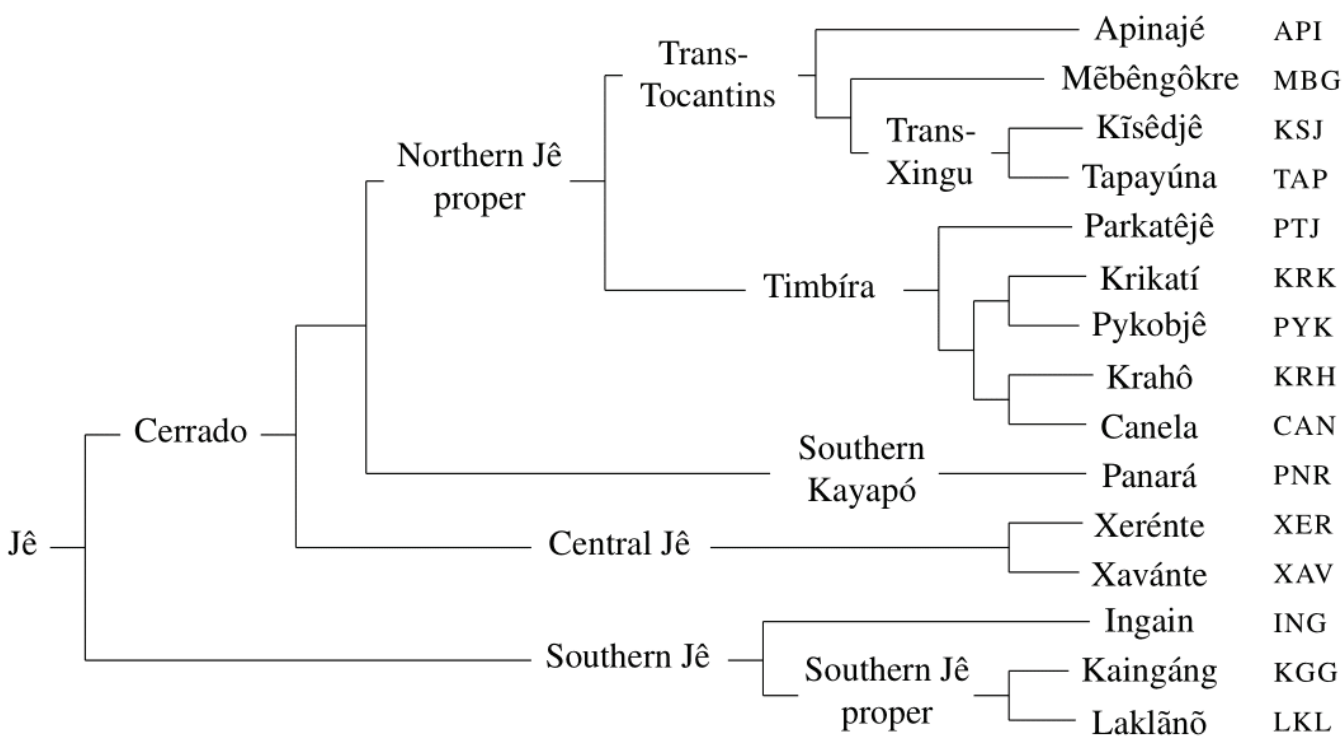

The remainder of this paper is organized as follows. Section 2 contains an outline of the phonological systems reconstructible to Proto-Cerrado (subsection 2.1) and Proto-Southern Jê (subsection 2.2). In section 3, I examine the sound correspondences between Proto-Cerrado and Proto-Southern Jê and come up with a reconstructive proposal for Proto-Jê. Onsets (subsection 3.1), nuclei (subsection 3.2), and codas (subsection 3.3) are dealt with separately. A list of the most reliable Jê etymologies is provided in section $\mathbf{4}$. Section $\mathbf{5}$ summarizes the paper and lists several unresolved issues in comparative Jê linguistics that should be subject to future research.

${ }^{2}$ One noteworthy example is the bias towards Finnic data in Uralic comparative studies, which prevented the scientific community from providing a correct account of several important vowel correspondences for decades; only very recently (Aikio 2012, 2015) did it become clear that Finnic vocalism is innovative in a number of respects. Another well known case was the over-reliance on Sanskrit data in mid-nineteenth century IndoEuropean comparative studies, which led scholars to a long-lasting misconception that Proto-Indo-European vocalism was identical to that of Sanskrit (Benware 1974: 66-81).

3 The tree in Figure 1 was initially obtained through the application of StarlingNJ, a distance-based lexicostatistical method (see Kassian 2015: 5 for details), to 110-item wordlists of 16 extant Jê varieties (including several dialects of Kaingáng and Mẽbêngôkre but excluding Krikatí and Ingain). The annotated wordlists are scheduled to be published online at <http://starling.rinet.ru/new100> in 2019 as a part of the Global Lexicostatistical Database. For each node identified by the StarlingNJ algorithm, shared lexical, phonological and/or morphosyntactic innovations can be presented. For reasons of space, I do not discuss the internal classification of Jê in what follows, hoping to revisit the subject in future publications. 


\section{Proto-Cerrado and Proto-Southern Jê}

In this section, I describe in detail the Proto-Cerrado (PCerr) and Proto-Southern Jê (PSJ) reconstructions adopted in this paper.

\subsection{Proto-Cerrado}

The Cerrado branch of the Jê family is constituted by two subbranches, Northern Jê $\hat{\mathrm{e}}^{4}$ and Central Jê. I use the reconstruction of Proto-Cerrado by Nikulin (2017), according to which the phonological inventory of this proto-language comprised 12 consonants and 15 vowels. PCerr consonantal phonemes are shown in Table 1 below.

Table 1. Proto-Cerrado consonants. ${ }^{5}$

\begin{tabular}{|r|c|c|c|c|}
\cline { 2 - 5 } \multicolumn{1}{c|}{} & labial & coronal & palatal & velar \\
\hline oral stops & ${ }^{*} \mathrm{p}$ & ${ }^{*} \mathrm{t}$ & ${ }^{*} \mathrm{c}$ & ${ }^{*} \mathrm{k}$ \\
\hline nasal stops & ${ }^{*} \mathrm{~m}$ & ${ }^{*} \mathrm{n}$ & ${ }^{*} \tilde{\mathrm{n}}$ & ${ }^{*} \mathrm{y}$ \\
\hline approximants & ${ }^{*} \mathrm{w}$ & ${ }^{*} \mathrm{r}$ & ${ }^{*} \mathrm{j}$ & ${ }^{*} \mathrm{u}^{6}$ \\
\hline
\end{tabular}

In oral environments, underlying nasal onsets were realized as postoralized $\left({ }^{*} m b-,{ }^{*} n d-,{ }^{*} n \hat{\jmath}^{-}\right.$, ${ }^{*} \eta g-$ ). In nasal environments, the contrasts ${ }^{*} j /{ }^{*} \tilde{n}$ and ${ }^{*} u /{ }^{*} \eta$ were neutralized in favor of ${ }^{*} \tilde{n}$ and ${ }^{*} \eta$, respectively. All these allophonic processes are reflected in the transcription system used in this work. The pronunciation of PCerr consonants in coda position is uncertain, but at least ${ }^{*} m$ is likely to have had a preoralized allophone ${ }^{*}[-\mathrm{b} \mathrm{m}]$ in oral environments (unless followed by an echo vowel, for which see below).

The 15 vowels of PCerr are listed in Table 2 below.

${ }^{4}$ For convenience, I will use "Northern Jê" and "Proto-Northern Jê" ("PNJ") as synonyms of "Northern Jê proper" and "Proto-Northern Jê proper" (that is, excluding Panará/Southern Kayapó) throughout this paper. I will refer to Northern Jê lato sensu as "Northern Jê-Panará” whenever necessary.

${ }^{5}$ In this work, I diverge from my earlier practice of representing linguistic data using the Unified Transcription System (UTS) and adhere to a modified version of the International Phonetic Alphabet (IPA) instead. The main differences between the transcription system used in this work and IPA are: $r=\operatorname{IPA} r ; \tilde{n}=\operatorname{IPA} n$; $c$ stands for any voiceless palatal, alveo-palatal or postalveolar stop, or affricate; $\hat{\jmath}$ stands for any voiced palatal, alveo-palatal or postalveolar stop or affricate; $x$ stands for a voiceless alveo-palatal or postalveolar fricative; $a, o, e$, $\tilde{a}, \tilde{o}, \tilde{e}$ are open-mid or simply mid; $\hat{\partial}, \hat{o}, \hat{e}$ are close-mid; $a, \hat{a}, x, y$ are central or back unrounded vowels (open-mid, close-mid, near-close, and close, respectively); $\hat{\imath}, \hat{u}, \hat{y}$ are falling lowering diphthongs. In Karajá data, the circumflex stands for the [+ATR] feature. The apostrophe in PCerr reconstructed forms is used to indicate that the echo vowel is absent (see Nikulin 2017: 168-169 on this phenomenon).

${ }^{6}$ Although the reflexes of this segment in all Cerrado languages are stops (MBG $g$, API, TIM, TAP, KSJ, PNR, XER $k$, XAV 2), it patterns phonologically with PCerr ${ }^{*} j$ and ${ }^{*} w$ in a number of respects (see Nikulin \& Salanova forthc. for discussion), suggesting that at least phonologically it belonged to the natural class of approximants in ProtoCerrado. Fortition of approximants, especially in stressed syllables, is a recurrent trend across Cerrado languages: the development ${ }^{*} w>{ }^{*} b$ is known from Proto-Northern Jê-Panará, and the development ${ }^{*} j>{ }^{*} \hat{j}$ is hypothesized for Proto-Cerrado (in all environments), for Proto-Northern Jê-Panará (in stressed syllables), and for Proto-TransTocantins (in unstressed syllables preceding ${ }^{*}{ }^{*}{ }^{*} u$ ). That way, it does not seem entirely impossible that the segment in question was an approximant not only phonologically, but also phonetically in Proto-Cerrado. 
Table 2. Proto-Cerrado vowels.

oral

\begin{tabular}{ccc}
\hline $\begin{array}{c}\text { front } \\
\text { unrounded }\end{array}$ & $\begin{array}{c}\text { non-front } \\
\text { unrounded }\end{array}$ & $\begin{array}{c}\text { back } \\
\text { rounded }\end{array}$
\end{tabular}

\begin{tabular}{|c|c|c|c|}
\hline close & $*_{i}$ & ${ }^{*} \mathrm{y}$ & ${ }^{*} \mathrm{u}$ \\
\hline close-mid & $* \hat{e}$ & $* \hat{\partial}$ & ${ }^{*} \hat{O}$ \\
\hline open-mid & ${ }^{*} \mathrm{e}$ & *ə & ${ }^{*} \mathrm{O}$ \\
\hline open & & $* a$ & \\
\hline
\end{tabular}

nasal

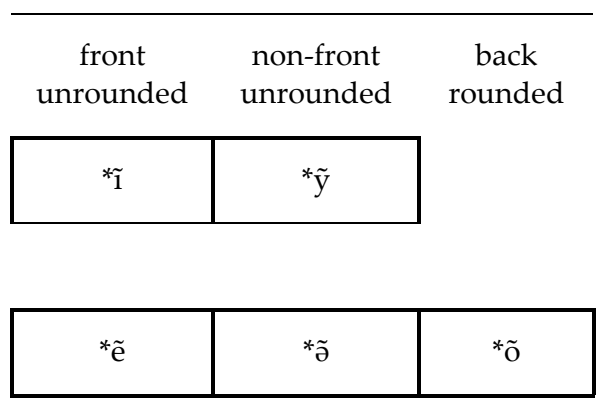

An already rich vocalic system reconstructible to Proto-Cerrado became even richer in ProtoNorthern Jê, where ${ }^{*} \tilde{a}$ and ${ }^{*} \tilde{u}$ emerged due to positional nasalization.

Apart from the monophthongs, at least two complex nuclei, ${ }^{*} w a$ and ${ }^{*} j a,{ }^{8}$ are reconstructible to Proto-Cerrado. Note that new important etymologies have been identified since the publication of the previous proposal (Nikulin 2017: 163), corroborating the reconstruction of the diphthongs in question. I list these etymologies in (1) below.

(1) New Cerrado etymologies involving a diphthong

a. PCerr ${ }^{*} c w a /{ }^{*} c w a-r$ ' 'to ask' $>$ PNJ ${ }^{*} c \hat{u} /{ }^{*} c w \hat{\partial}-r$, PCJ ${ }^{*} w a /{ }^{*} w a-r i$

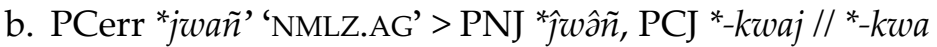

c. PCerr ${ }^{*} j a j w a /{ }^{*} j a j w a-r$ ' 'to lay.PL, to spill' > PNJ ${ }^{*} j a \hat{\jmath} \hat{u} /{ }^{*} j a \hat{\jmath} w \hat{\partial}-r, \mathrm{PCJ}{ }^{*} c-a(j) k w a /{ }^{*} c-a(j) k w a-r i$

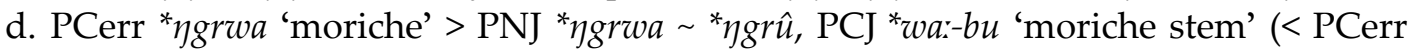
$\left.{ }^{*} \eta g r w a-p u>P N J{ }^{*} \eta g r w a-p u\right)$

e. PCerr * $\tilde{n} \tilde{o} w w a$ 'sternum'> PNJ * $\tilde{n} \tilde{o} k w a, \mathrm{PCJ}$ * $\tilde{n} \tilde{w} w a$ 'in front of'

f. PCerr ${ }^{*} \tilde{n} \tilde{o} r k w a$ 'home' > PNJ * $\tilde{n} \tilde{u} r k w a$, PCJ ${ }^{*} \tilde{n} \tilde{o} r \tilde{w} w a$

g. PCerr *krwat 'beak'> PNJ *krwât, PCJ *wata // *wada

h. PCerr * ${ }^{*} w a j-c i$ 'rib' > PNJ * rwồ-ci, PCJ *waj-hi

i. PCerr *rwaj' 'moon' > PNJ *mbyt-rwâ, PCJ *waj // *wa

j. PCerr *twam' 'fat' > PNJ *twâm, PCJ *wam // *wa

k. PCerr ${ }^{*} a-\eta g j a /{ }^{*} \eta g j a-c$ 'to enter.PL' > PNJ ${ }^{*} a-\eta g \hat{\imath} \sim{ }^{*} a-\eta g j \hat{e} /{ }^{*} \eta g j \hat{e}-c$, PCJ * $\tilde{\jmath}-\hat{\jmath} a /{ }^{*} \hat{\jmath} a-c i$ 'to enter.DU' 9

1. PCerr ${ }^{*} \eta g j a /{ }^{*} \eta g j a-\tilde{n}$ ' 'to enter.CAUS.PL' > PNJ ${ }^{*} \eta g \hat{\imath} \sim{ }^{*} \eta g j \hat{e} /{ }^{*} \eta g j \hat{e}-\tilde{n}$, PCJ ${ }^{*} \hat{\jmath} a j / /{ }^{*} \hat{\jmath} a$ 'to enter.CAUS.DU'

m. PCerr ${ }^{*} k r j a{ }^{*} k r j a-r$ ' 'to bring up' > PNJ ${ }^{*} k r \hat{\imath} /{ }^{*} k j \hat{e}-r$, PCJ ${ }^{*} \hat{\jmath} a{ }^{*} \hat{\jmath} a-r i$

${ }^{7}$ It is uncertain whether non-front unrounded vowels were phonetically central or back.

${ }^{8}$ Nikulin (2017) adopted the reconstruction *jê. The change in reconstruction is suggested by the nearsymmetry of the reflexes of PCerr ${ }^{*} j a$ and ${ }^{*} w a$ in daughter languages. In Proto-Northern Jê, these two diphthongs yielded *jê and *wâ in closed syllables (as well as in open syllables that go back to PCerr syllables ending in * $j$ ), but ${ }^{*} \hat{e} \sim{ }^{*} \hat{\imath}$ and ${ }^{*} w a \sim{ }^{*} \hat{u}$ in originally open syllables (with a so far unclear variation that is sometimes preserved in Proto-Northern Jê, see Nikulin 2017: 178-179). In Proto-Central Jê, the reflexes of these diphthongs are * $\hat{\jmath} a$ and *wa, and any PCerr onset is eliminated before the diphthong (except that PCerr *jwa > PCJ *kwa).

9 The etymologies (1k) and (11) had been erroneously conflated in Nikulin 2017. Although related, they are two clearly distinct verbs. The same semantic and morphological relation holds for their singular counterparts:

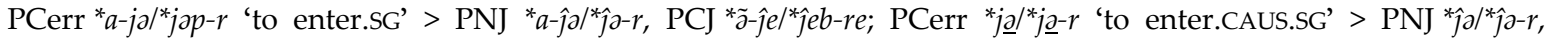
PCJ ${ }^{*} \tilde{n} \tilde{e} /{ }^{*} \tilde{n} \tilde{e}-r \tilde{e}$. 
n. (?) PCerr *kwa/kwa-r' 'to crack nuts' > PNJ *kukwâl/*ukwôे-r, PCJ *wa/*wa-ri

o. (?) PCerr *krwaj 'parrot' > PNJ *krwâj, PCJ *waj // *wa, *waj-hara

In Proto-Cerrado, the onset position could be filled with any single consonant or by a cluster formed by a peripheral (labial or velar) stop and ${ }^{*}-r-:{ }^{*} p r,{ }^{*} m(b) r,{ }^{*} k r,{ }^{*} \eta(g) r$. Strikingly, the occurrence of onsetless syllables appears to have been very limited: only pretonic syllables that contained ${ }^{*} a$ or ${ }^{*} i$ as its nucleus could lack a consonantal onset.

Syllables could also have a coda in Proto-Cerrado, which could be followed or not by a so called echo vowel (only word-finally). In my notation, the occurrence of an echo vowel after a consonantal coda is unmarked, whereas the absence of a word-final echo vowel is marked with the apostrophe. The following codas can be reconstructed for Proto-Cerrado: ${ }^{*} p,{ }^{*} t,{ }^{*} c,{ }^{*} k$, ${ }^{*} m,{ }^{*} n,{ }^{*} r,{ }^{*} j,{ }^{*} p r,{ }^{*} m$ ', ${ }^{*}{ }^{\prime},{ }^{*} \tilde{n},{ }^{*} r{ }^{*},{ }^{*} j$ ', ${ }^{*} p r$ '. The complex codas ${ }^{*} p r$ and ${ }^{*} p r$ ' occur exclusively in non-finite verb forms, when the non-finite suffix ${ }^{*}-r$ is adjoined to a verbal stem that ends in an underlying labial consonant. Very few examples are available that could substantiate the reconstruction of Proto-Cerrado ${ }^{*} C,{ }^{*} n,{ }^{*} \eta,{ }^{*} j,{ }^{*} n$, ${ }^{*} \tilde{n}$ ' in coda position. ${ }^{10}$ The reconstruction of Proto-Cerrado ${ }^{*} d$, as proposed by Nikulin (2017: 169), is even more problematic in that no ${ }^{*} d$ can be reconstructed for the onset position.

One major issue in Cerrado historical phonology remains entirely unresolved. Namely, some Central Jê stems unexpectedly contain nasal nuclei in stems whose cognates in Northern Jê (and in other Macro-Jê languages, whenever available) display oral nuclei. Examples include PCJ ${ }^{*} \tilde{n} \tilde{e} /{ }^{*} \tilde{\hat{e}} \tilde{-}-r \tilde{e}$ 'to enter.CAUS.SG' (cf. PNJ $\left.{ }^{*} \hat{\jmath} \partial{ }^{*} \hat{\jmath} \partial-r\right),{ }^{*} c-\tilde{\partial} /{ }^{*} c-\tilde{\partial}-r \tilde{\imath}$ 'to stand.CAUS.SG'

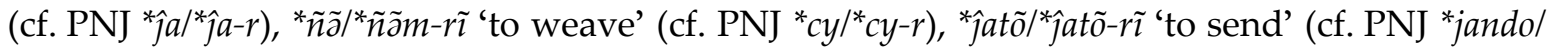
${ }^{*} j a n d o-r$ ), ${ }^{*} c-a c \tilde{o} /{ }^{*} c-a c o ̃ m-r \tilde{\imath}$ 'to hang' (cf. PNJ $\left.{ }^{*} j a n \hat{\jmath} \hat{o} /{ }^{*} j a n \hat{\jmath} \hat{o}-r\right),{ }^{*} r \tilde{e} /{ }^{*} r \tilde{e}-m \tilde{e}$ 'to leave.SG' (cf. PNJ ${ }^{*} r e$ ),

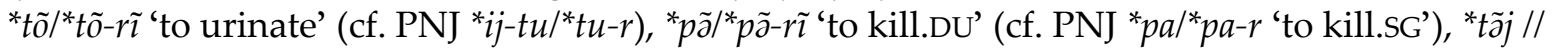
*tã 'rain' (cf. PNJ *nda). The origins of nasalization in Central Jê have not yet been identified, and it is uncertain how the respective PCerr forms should be reconstructed. I leave this question open and provisionally represent these unclear cases by underlining the nuclei in my

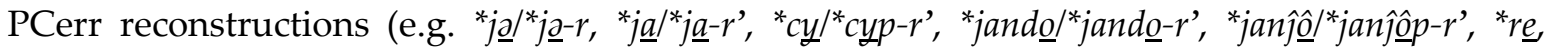

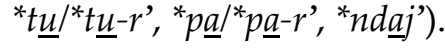

\subsection{Proto-Southern Jêe 11}

The reconstruction of PSJ consonantism does not pose serious difficulties, and the systems reconstructed by Wiesemann (1978) and Jolkesky (2010) are almost identical. I accept their reconstruction with minor changes in notation.

Although underlyingly nasal, voiced stops were subject to postoralization in oral environments ( $\left.{ }^{*} m b-,{ }^{*} n d-,{ }^{*} n \hat{\jmath}^{-},{ }^{*} \eta g-\right)$; this allophony pattern is active in both Southern Jê languages and is reconstructible to Proto-Cerrado. The preoralization of underlying nasal codas is also attested both in Kaingáng and Laklãnõ, though this process is not equally robust in all Southern Jê varieties: depending on the dialect and on the place of articulation, either the oral phase or the nasal phase may be omitted. Although clearly reconstructible to Proto-Southern Jê, I chose not to represent this allophony in my transcription.

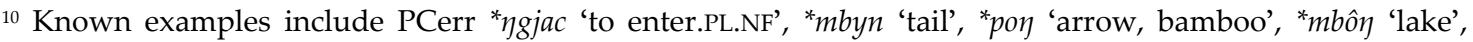

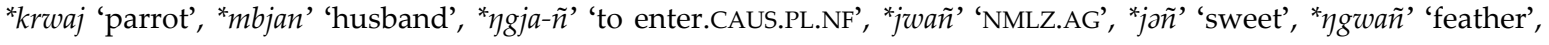
maybe also *mbec 'good' and *mbên 'liquid'.

${ }^{11}$ In this paper, I do not consider Ingain data due to their ill-transcribed nature. Hereinafter the terms "Southern Jê" and "Proto-Southern Jê" are used in their narrow definition (that is, "Southern Jê proper" and "Proto-Southern Jê proper", excluding Ingain). Ingain data will need to be subjected to the procedure of restitution (Constenla Umaña 2000) before they can be meaningfully used in the reconstruction of Proto-Jê. 
Table 3. Proto-Southern Jê consonants.

\begin{tabular}{|c|c|c|c|c|c|}
\hline & labial & coronal & palatal & velar & glottal \\
\hline oral stops & ${ }^{*} \mathrm{p}$ & $*_{t}$ & ${ }^{*} \mathrm{C}$ & ${ }^{*} \mathrm{k}$ & $* ?$ \\
\hline fricative & & ${ }^{*} \theta$ & & & \\
\hline nasal stops & ${ }^{*} \mathrm{~m}$ & ${ }^{*} \mathrm{n}$ & ${ }^{*} \tilde{\mathrm{n}}$ & $*^{*} y$ & \\
\hline approximants & ${ }^{*} \mathrm{~W}$ & ${ }^{*} \mathrm{r}$ & ${ }^{*} j$ & & ${ }^{*} \mathrm{~h}$ \\
\hline
\end{tabular}

Regarding the vocalic system of Proto-Southern Jê, I have previously argued (Nikulin 2015) that Jolkesky's (2010) reconstruction of PSJ vowels is seriously flawed and put forward a proposal backed up with morphophonological evidence. Note that while my reconstruction of PSJ oral vowels coincides with that of Wiesemann (1978), our reconstructions of PSJ nasal vowels are radically different. ${ }^{12}$

Table 4. Proto-Southern Jê vowels.

oral

front un-
rounded

$$
\text { non-front }
$$
unrounded

back rounded

\begin{tabular}{|c|c|c|c|}
\hline close & $*_{i}$ & ${ }^{*} \mathrm{y}$ & ${ }^{*} \mathrm{u}$ \\
\hline close-mid & *ê & $* \hat{\partial}$ & ${ }^{*} \hat{O}$ \\
\hline open-mid & ${ }^{*} \mathrm{e}$ & *ə & ${ }^{*} \mathrm{O}$ \\
\hline open & & $* a$ & \\
\hline
\end{tabular}

nasal

\begin{tabular}{|ccc|}
\hline $\begin{array}{c}\text { front un- } \\
\text { rounded }\end{array}$ & $\begin{array}{c}\text { non-front } \\
\text { unrounded }\end{array}$ & $\begin{array}{c}\text { back } \\
\text { rounded }\end{array}$ \\
& & $*_{\tilde{1}}$ \\
& &
\end{tabular}

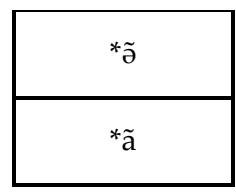

The phonotactic restrictions are identical in Kaingáng and Laklãnõ and are easily traced back to Proto-Southern Jê. The maximal syllable structure reconstructible to PSJ is ${ }^{*} \mathrm{CrVC}$. Just like in Proto-Cerrado, only labial and velar stops could form complex onsets with the rhotic: ${ }^{*} p r,{ }^{*} m(b) r,{ }^{*} k r,{ }^{*} \eta(g) r$. As for the codas, only voiced segments could occur in that position; approximant codas were obligatorily followed by a non-phonemic echo vowel (phonetically a copy of the nucleus), whereas nasal codas did not trigger the occurrence of any vocalic segment. I leave PSJ echo vowels unmarked in my transcription because their occurrence and quality are fully predictable.

12 Jolkesky (2010: 173) reconstructs yet another nasal vowel for Proto-Southern Jê, tracing the correspondence KGG $\tilde{a} \sim$ LKL $\tilde{u}$ back to PSJ * $\tilde{y}$ ( ${ }^{*} \tilde{u}$ in his transcription). Nikulin (2015: 286) reinterprets it as * $\tilde{o}\left({ }^{*} \tilde{\jmath}\right.$ in the transcription of the original paper). Note, however, that this sound correspondence occurs exclusively in grammatical

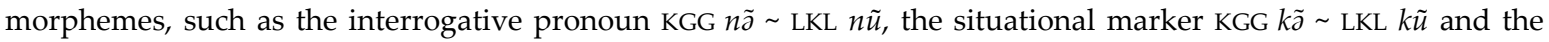
subject marker KGG wã $\sim$ LKL $v \tilde{u}$. It now seems possible that this sound correspondence may have arisen as a

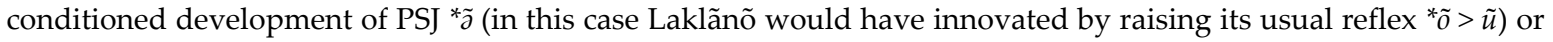
PSJ ${ }^{*} \tilde{u}$ (in this case Kaingáng would have innovated by unrounding and lowering its usual reflex ${ }^{*} \tilde{u}>\tilde{a}$ ) in unstressed grammatical morphemes. 


\section{Proto-Jê phonology}

In this section, I examine the correspondences between Proto-Cerrado and Proto-Southern Jê onsets (3.1), nuclei (3.2), and codas (3.3), offering in each case a Proto-Jê reconstruction. Some Proto-Jê elements appear to have been preserved in only one constituent branch; in such cases, external evidence from other Macro-Jê languages has also been discussed.

\subsection{Onsets}

The correspondences between the onsets reconstructible for PCerr and PSJ are mostly trivial except for the palatal place of articulation, as shown in Table 5.

Table 5. Proto-Jê onsets and their reflexes in Proto-Cerrado and Proto-Southern Jê.

\begin{tabular}{|c|c|c|c|c|c|}
\hline \multirow{2}{*}{\multicolumn{2}{|c|}{$\begin{array}{c}\text { Proto-Jê } \\
{ }^{*} p\end{array}$}} & \multicolumn{2}{|c|}{ Proto-Cerrado } & \multicolumn{2}{|c|}{ Proto-Southern Jê } \\
\hline & & $* / \mathrm{p} /$ & ${ }^{*} p$ & $* / \mathrm{p} /$ & ${ }^{*} p$ \\
\hline$* / \mathrm{m} /$ & ${ }^{*} m b,{ }^{*} m$ & $* / \mathrm{m} /$ & ${ }^{*} m b,{ }^{*} m$ & $* / \mathrm{m} /$ & ${ }^{*} m b,{ }^{*} m$ \\
\hline$* / \mathrm{w} /$ & ${ }^{*} w$ & $* / \mathrm{w} /$ & ${ }^{*} w$ & $* / \mathrm{w} /$ & ${ }^{*} w$ \\
\hline$* / p r /$ & ${ }^{*} p r$ & $* / p r /$ & ${ }^{*} p r$ & $* / \mathrm{pr} /$ & *pr \\
\hline$* / \mathrm{mr} /$ & ${ }^{*} m b r,{ }^{*} m r$ & $* / \mathrm{mr} /$ & ${ }^{*} m b r,{ }^{*} m r$ & $* / \mathrm{mr} /$ & ${ }^{*} m b r,{ }^{*} m r$ \\
\hline$* / t /$ & ${ }^{*} t$ & $* / t /$ & ${ }^{*} t$ & $* / t /$ & $* t$ \\
\hline$* / \mathrm{n} /$ & ${ }^{*} n d,{ }^{*} n$ & $* / \mathrm{n} /$ & ${ }^{*} n d,{ }^{*} n$ & $* / \mathrm{n} /$ & ${ }^{*} n d,{ }^{*} n$ \\
\hline$* / \mathrm{r} /$ & ${ }^{*} r$ & $* / \mathrm{r} /$ & ${ }^{*} r$ & $* / \mathrm{r} /$ & ${ }^{*} r$ \\
\hline$* / \mathrm{c} /$ & ${ }^{*} \mathrm{C}$ & $* / \mathrm{c} /$ & $*_{C}$ & $* / \theta /$ & ${ }^{*} \theta$ \\
\hline$* / \tilde{\mathbf{n}} /$ & ${ }^{*} n \hat{\jmath}$ & $* / \tilde{n} /$ & ${ }^{*} n \hat{\jmath}$ & $* / \mathrm{c} /$ & ${ }^{*} \mathrm{C}$ \\
\hline \multirow{2}{*}{$* / \mathrm{j} /$} & ${ }^{*} j$ & \multirow{2}{*}{$* / \mathrm{j} /$} & ${ }^{*} j$ & $* / j /$ & ${ }^{*} j$ \\
\hline & ${ }^{*} \tilde{n}$ & & $* \tilde{n}$ & $* / \mathrm{n} /$ & ${ }^{*} n$ \\
\hline$* / \mathrm{k} /$ & ${ }^{*} k$ & $* / \mathrm{k} /$ & *k & $* / \mathrm{k} /$ & ${ }^{*} k$ \\
\hline$* / \mathrm{y} /$ & ${ }^{*} \eta g,{ }^{*} \eta$ & $* / \mathrm{y} /$ & $* \eta g, * \eta$ & $* / \mathrm{y} /$ & ${ }^{*} \eta g, * \eta$ \\
\hline$* / \mathrm{kr} /$ & $* k r$ & $* / \mathrm{kr} /$ & ${ }^{*} k r$ & $* / \mathrm{kr} /$ & ${ }^{*} k r$ \\
\hline$* / \mathrm{pr} /$ & ${ }^{*} \eta g r,{ }^{*} \eta r$ & $* / \mathrm{nr} /$ & ${ }^{*} \eta g r,{ }^{*} \eta r$ & $* / \mathrm{pr} /$ & ${ }^{*} \eta g r,{ }^{*} \eta r$ \\
\hline$* / \varnothing /$ & ${ }^{*} \varnothing(?)$ & $* / \mathrm{uq} /$ & ${ }^{*} u,{ }^{*} \eta$ & $?$ & $?$ \\
\hline
\end{tabular}


Most of the correspondences in Table 5 are identity correspondences, thus posing no difficulty for the reconstruction of the respective Proto-Jê segments. For reasons of space, the identity correspondences between Proto-Cerrado and Proto-Southern Jê onsets will not be examined in this section; numerous examples are available in Section 4.

Regarding the origin of the infrequent PCerr ${ }^{*} w$, it is unknown which segment should be reconstructed to Proto-Jê due to the fact that the tokens that contain this phoneme in ProtoCerrado lack known cognates in Southern Jê. Nevertheless, it is certain that these tokens are of Jê origin, given that they have secure external cognates in other Macro-Jê languages, where PCerr ${ }^{*} \psi$ corresponds to glottal segments (Maxakalí $h$; Krenák $h$; Jabutí 2 ; cf. Nikulin \& Silva, forthc.). For lack of a better solution, I reconstruct ${ }^{*} \varnothing\left(>\right.$ PCerr $\left.{ }^{*} u\right)$ for Proto-Jê, but it should be kept in mind that other possibilities (e.g. ${ }^{*} y$ or ${ }^{*} h$ ) cannot be totally ruled out at present. Note that not a single onsetless monosyllabic root morpheme reconstructs to Proto-Cerrado, making it possible to stipulate an epenthetical origin for PCerr ${ }^{*} u$.

Palatal onsets. I claim that Proto-Cerrado is conservative with respect to the palatal onsets of Proto-Jê. Conversely, Proto-Southern Jê would have undergone a chain shift: as shown in (2-3) below, PJ ${ }^{*} n \hat{\jmath}>$ PSJ ${ }^{*} C$, whereas $\mathrm{PJ}{ }^{*} \mathrm{C}>{ }^{*} \theta$.

(2) Proto-Jê ${ }^{*} n \hat{\jmath}>$ Proto-Southern Jê ${ }^{*} C$

a. PJ ${ }^{*} n \hat{\jmath} y$ 'bee, bumblebee' > PCerr ${ }^{*} n \hat{\jmath} y$, PSJ ${ }^{*} c y$

b. PJ " $n \hat{\jmath} \hat{o}$ 'to hang.SG'> PCerr ${ }^{*} n \hat{j} \underline{\hat{o}} /{ }^{*} n \hat{j} \hat{o} p-r$ ', PSJ ${ }^{*} c a /{ }^{*} c a-\eta\left(\mathrm{vb} .{ }^{*} c \partial-m\right)$

c. PJ * $n \hat{\jmath} u$ 'to heat, to dry'> PNJ ${ }^{*} n \hat{\jmath} u /{ }^{*} n \hat{\jmath} u-\tilde{n}$ 'to dry in the sun'; PSJ ${ }^{*} \mathrm{Cu}-r\left(\mathrm{vb} .{ }^{*} \mathrm{Cu}-n\right)$ 'to heat by the fire', ${ }^{*} j a \eta-c u /{ }^{*} j a \eta-c u-\eta,{ }^{*} j a \eta-c u-r\left(v b .{ }^{*} j a \eta-c u-n\right)$ 'to roast'

d. PJ * $n \hat{\jmath} \hat{e}$ 'to tickle' > PNJ ${ }^{*} n \hat{\jmath} \hat{e} /{ }^{*} n \hat{\jmath} \hat{e}-r$, PSJ ${ }^{*} k u-c \hat{e} l^{*} k u-c e-\eta$

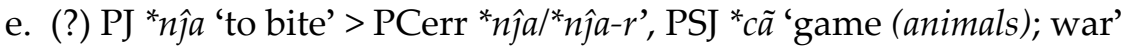

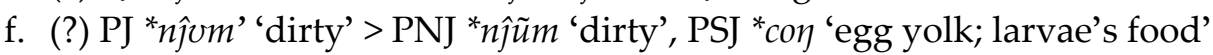

g. (?) PJ * $n \hat{\imath} i$ 'mother'> PNJ * $n \hat{j} i$, PSJ * $c i$ 'old'

(3) Proto-Jê ${ }^{*} c>$ Proto-Southern Jê ${ }^{*} \theta$
a. PJ *cy 'to weave' > PCerr * $c y /{ }^{*} c y p-r$ ', PSJ ${ }^{*} \theta y$
b. PJ *cym 'seed'> PCerr ${ }^{*} c y m$, PSJ ${ }^{*} \theta y\left(v b .{ }^{*} \theta y-n\right)$
c. PJ “côj 'leaf'> PCerr * côj', PSJ ${ }^{*} \theta e j\left(\mathrm{vb} .{ }^{*} \theta e-\tilde{n}\right)$

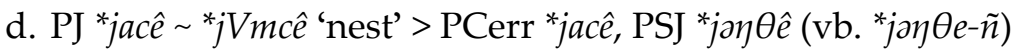
e. PJ ${ }^{*}-\tilde{\imath}(C)$ 'bandage, sling' $>$ PNJ ${ }^{*} j a-c \tilde{\imath},{ }^{*} k a-c \tilde{l}$, PSJ ${ }^{*}-\theta \tilde{\imath}($ vb. $-\theta \tilde{\imath}-n)$

Note that $\mathrm{PJ}{ }^{*} \mathrm{C}$ - is also reconstructible as the allomorph of the third person marker used with *j-initial stems. As suggested by Ribeiro (2004: 95-96, 2011: 109, fn. 8), Proto-Southern Jê appears to have fossilized an earlier third person prefix ${ }^{*} \mathrm{C}$ - in some stems. ${ }^{13}$

f. PJ ${ }^{*} j \hat{j}, 3$ (= third person) ${ }^{*} c-\hat{a}$ 'bitter' $>$ PCerr ${ }^{*} j \hat{\partial}$, PSJ ${ }^{*} \theta a\left(v b .{ }^{*} \theta a-\eta\right)$

h. PJ *jara, $3{ }^{*}$ c-ara 'wing; armpit' > PNJ *jara 'wing', *jara-kre 'armpit', PSJ * $\theta \tilde{a} r$ 'wing' (but *jãrã 'armpit', vb. ${ }^{*} j \tilde{a} r \tilde{a}-n$ )

${ }^{13}$ It is worth observing that the stems that underwent this fossilization process are those that typically have an inanimate internal argument. This semantic peculiarity may have contributed to an elevated frequency of occurrences of these stems with a third person prefix in Proto-Jê discourse when compared to other ${ }^{*} j$-initial stems, thus enabling the reanalysis. Another comment to be made is that this fossilization trend continued in Kaingáng even after the dissolution of Proto-Southern Jê: cf. PJ ${ }^{*} j i, 3{ }^{*} c-i$ 'to lay.SG' > PSJ ${ }^{*} j i, 3{ }^{*} \theta-i>$ KGG $\phi i$, LKL $j i, 3 \partial-i$. In other instances, on the contrary, Kaingáng generalized the uninflected form: PSJ *jãmã- $\eta, 3$ * $\theta$-ãmã- $\eta$ ‘to listen'>

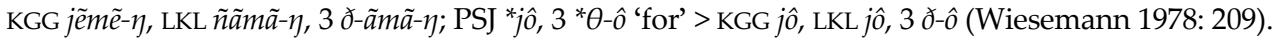


i. PJ ${ }^{*} j v, 3{ }^{*} c-v$ 'pus' $>$ PCerr ${ }^{*} j u p-r$, PSJ ${ }^{*} \theta \hat{o}\left(v b .{ }^{*} \theta o-m\right)$

j. PJ ${ }^{*} j \hat{e}, 3{ }^{*} c-\hat{e}\left(\sim{ }^{*} j e \hat{e} n, 3 c-\hat{e} n\right)$ 'thread, knot, cloth'> PNJ ${ }^{*} \hat{\jmath} \hat{e}$, PSJ vb. ${ }^{*} \theta e-n$ 'to spin thread'

k. PJ ${ }^{*} j a, 3{ }^{*} c-a$ 'to put vertically.SG' > PCerr ${ }^{*} \underline{j}{ }^{*}{ }^{*} \underline{a}-r$ ', PSJ ${ }^{*} \theta \tilde{a}\left(\mathrm{vb} .{ }^{*} \theta \tilde{a}-\eta\right)$ 'to be put vertically'

In addition, Proto-Southern Jê depalatalized PJ ${ }^{*} \tilde{n}$ (the nasal allophone of ${ }^{*} / \mathrm{j} /$ ) to PSJ ${ }^{*} n$, merging it with PSJ ${ }^{*} n<$ PJ ${ }^{*} n$, as shown in (4) below.

(4) Proto-Jê ${ }^{*} \tilde{n}>$ Proto-Southern Jê ${ }^{*} n$

a. PJ * $\tilde{n} \tilde{\imath}$ 'meat' > PCerr ${ }^{*} \tilde{n} \tilde{\imath}$, PSJ ${ }^{*} n \tilde{\imath}$

b. PJ * $\tilde{n} \tilde{y}$ 'to sit.SG' > PCerr ${ }^{*} \tilde{n} \tilde{y} /{ }^{*} \tilde{n} \tilde{y} p-r$, PSJ ${ }^{*} n \tilde{\imath}\left(\mathrm{vb} .{ }^{*} n \tilde{\imath}-m\right)$

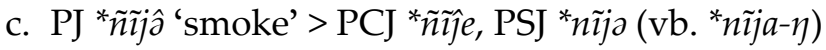

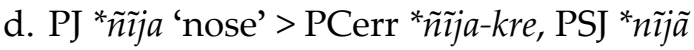

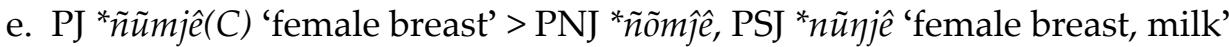

f. PJ * ${ }^{*} t{ }^{*} \tilde{n} \tilde{u} t$ 'to sleep' > PCerr * $\eta \tilde{o} t /{ }^{*} \tilde{n} \tilde{o} t$, PSJ ${ }^{*} n \tilde{u} r$

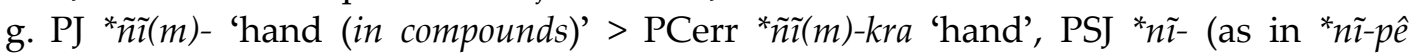
'to wash hands')

The fact that Proto-Cerrado retains the original configuration of the inventory at the palatal point of articulation is suggested by the typology of the directionality of sound changes (from a cross-linguistic perspective, it is more plausible to posit a fricativization development such as ${ }^{*} C>{ }^{*} \theta$ than an unconditional fortition of a fricative), as well as by external data from Maxakalí, Krenák, and Karajá, in which languages PJ ${ }^{*} c$ corresponds to stops or affricates (Maxakalí ţ; Krenák kj; Karajá $d$, palatalized tf; cf. Nikulin \& Silva, forthc.).

Word-medial clusters. Sometimes voiced segments are found in Proto-Southern Jê stems where, judging by their Proto-Cerrado cognates, voiceless consonants would be expected. I hypothesize that these apparently aberrant correspondences instantiate a sound law according to which voiceless stops became voiced $\left(* / t, c />^{*} / n, j /\right)$ Proto-Southern Jê if preceded by an etymological coda (the coda itself may disappear). This rule ceased to be productive and no longer applies in new formations (compounds, reduplicated plurals etc.). Some examples are provided in (5).

(5) Voiceless consonants yield nasals in clusters in Proto-Southern Jê
a. PJ * $\tilde{n} \tilde{u}$ cta 'tongue' > PCerr ${ }^{*} \tilde{n} \tilde{o} j$ to, PSJ ${ }^{*} n \tilde{u} n \tilde{a}$

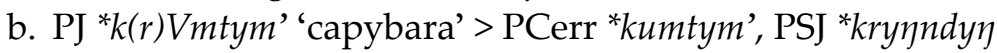
c. PJ *kapnja 'to chew' > PCerr *kapnjal*kapnja-r', PSJ *kajã
d. PJ ${ }^{*} n \hat{e} \hat{e}$ 'to tickle' > PNJ ${ }^{*} n \hat{\jmath} /{ }^{*} n \hat{\jmath} \hat{e}-r$, PSJ ${ }^{*} k u-c \hat{e} /{ }^{*} k u-c e-\eta$, pl. ${ }^{*} k u-\eta-j \hat{e} /{ }^{*} k u-\eta-j e-\eta$

In a number of instances, it is unknown whether the Southern Jê words have a Jê etymology, but alternations attested in derived forms appear to stem from the same diachronic process:

e. PSJ *ku-cir (vb. *ku-cin) 'to be roasted in fire' $\rightarrow$ pl. ${ }^{*} k u-\eta-j i r\left(v b .{ }^{*} k u-\eta-j i-n\right)$

f. PSJ *ku-côr (vb. $\left.{ }^{*} k u-c o n\right)$ 'to be pierced' $\rightarrow$ pl. ${ }^{*} k u-\eta-j o ̂ r(v b . ~ * k u-\eta-j o-n)$

g. PSJ *cin 'to glean' $\rightarrow$ antipassive *joy-jin

h. PSJ 'c $c \hat{e}{ }^{*} c e-\eta$ 'to tie' $\rightarrow{ }^{*} k a \eta-j \hat{e} /{ }^{*} k a \eta-j e-\eta$, pl. ${ }^{*} k y \eta-j \hat{e} /{ }^{*} k y \eta-j e-\eta$ 'to tie, to make a knot'; ${ }^{*} j y \eta-j \hat{e} /{ }^{*} j y \eta-j e-\eta$ 'to mend clothes'; ${ }^{*} \tilde{\eta} \eta-j \hat{e} /{ }^{*} \mathrm{NP} j \partial \eta-j \hat{e}$ 'trap with a maize bait', (?) *krĩ $\left.j \hat{e}\right|^{*} k r i \tilde{\eta}-j e-\eta$ 'to scratch; to go berserk' 


\subsection{Nuclei}

The correspondences between PCerr and PSJ nuclei are significantly less straightforward than those between the onsets of these reconstructed languages. They are listed in Table 6 below; tentative reconstructions of Proto-Jê segments are included.

Table 6. Proto-Jê nuclei and their reflexes in Proto-Cerrado and Proto-Southern Jê. ${ }^{14}$

\begin{tabular}{|c|c|c|}
\hline PJ & PCerr & PSJ \\
\hline
\end{tabular}$\quad$\begin{tabular}{|l|l|l|l|l|l|}
\hline PJ & PCerr & PSJ \\
\hline
\end{tabular}

\begin{tabular}{|c|c|c|}
\hline$*_{\mathrm{e}}$ & $*_{\mathrm{e}}$ & $*_{\mathrm{e}}$ \\
\hline$*_{\mathrm{e}}$ & $*^{*} \mathrm{e}$ & $*^{*} \mathrm{e}$ \\
\hline$*_{\mathrm{I}}$ & $*_{\mathrm{j} a}$ & $*^{*} \mathrm{e}$ \\
\hline$*_{\mathrm{i}}$ & $*_{\mathrm{i}}$ & $*_{\mathrm{i}}$ \\
\hline
\end{tabular}

\begin{tabular}{|c|c|c|}
\hline${ }^{*} a$ & $* a$ & *ã \\
\hline${ }^{*} \partial$ & ${ }^{*} \mathrm{O}$ & $* \tilde{a}$ \\
\hline${ }^{*} \hat{\partial}$ & $* \hat{\partial}$ & *ə \\
\hline${ }^{*} Y$ & $*_{\partial}$ & ${ }^{*} \hat{\partial}$ \\
\hline${ }^{*} \mathrm{y}$ & ${ }^{*} \mathrm{y}$ & ${ }^{*} \mathrm{y}$ \\
\hline
\end{tabular}

\begin{tabular}{|c|c|c|}
\hline${ }^{*} \mathrm{o}$ & ${ }^{*}$ wa & ${ }^{* a}$ \\
\hline${ }^{*} \mathrm{o}$ & ${ }^{*} \hat{\mathrm{o}}$ & ${ }^{*} \mathrm{\partial}$ \\
\hline${ }^{*} \mathrm{v}$ & ${ }^{*} \mathrm{u}$ & ${ }^{*} \mathrm{o}$ \\
\hline${ }^{*} \mathrm{u}$ & ${ }^{*} \mathrm{u}$ & ${ }^{*} \mathrm{u}$ \\
\hline
\end{tabular}

\begin{tabular}{|c|c|c|}
\hline$* \tilde{\mathrm{e}}$ & $* \tilde{\mathrm{e}}$ & $*_{\tilde{1}}$ \\
\hline$*_{\tilde{1}}$ & $*_{\tilde{1}}$ & $*_{\tilde{1}}$ \\
\hline
\end{tabular}

\begin{tabular}{|c|c|c|}
\hline$* \tilde{\partial}$ & $* \tilde{\partial}$ & $* \tilde{\partial}$ \\
\hline$* \tilde{y}$ & $*^{*} \tilde{y}, \tilde{\partial}^{\dagger}$ & $* \tilde{1}$ \\
\hline
\end{tabular}

\begin{tabular}{|c|c|c|}
\hline$* \tilde{\mathrm{o}}$ & $* \tilde{\mathrm{o}}$ & $* \tilde{\partial}$ \\
\hline$* \tilde{\mathrm{u}}$ & $* \tilde{\mathrm{o}}$ & $* \tilde{u}$ \\
\hline
\end{tabular}

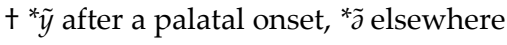

As can be seen from Table 6, I reconstruct a system with five contrastive vowel heights for Proto-Jê, in contrast with the systems of Proto-Cerrado and Proto-Southern Jê with only four vowel heights. Although systems with five contrastive heights are cross-linguistically rare (Ladefoged \& Maddieson 1990: $95-96),{ }^{15}$ it is of notice that one modern Macro-Jê language, Karajá, has also been documented to have five contrastive vowel heights (Ribeiro 2012: 86), though in featural terms Karajá vowel heights are better understood as an interaction between a ternary height contrast and a privative [ATR] feature.

If this reconstruction is accepted, the evolution of PCerr and PSJ nuclei can be described in the following way.

- From Proto-Jê to Proto-Cerrado (ordering uncertain):

(a) chain shift: ${ }^{*} Y>{ }^{*} \partial ;{ }^{*} \partial>{ }^{*} 0$; ${ }^{*} O{ }^{*} w a$;

(b) diphthongization of ${ }^{*} I>{ }^{*} j a$;

(c) raising of ${ }^{*} v>{ }^{*} u$;

(d) lowering of ${ }^{*} \tilde{y}>{ }^{*} \tilde{a}$ (except after palatals) and of ${ }^{*} \tilde{u}>^{*} \tilde{o}$ (unconditionally).

${ }^{14}$ I remind the reader that the transcription system adopted here differs from IPA in important ways (see fn. 5). Most importantly, the characters $<y, y, \tilde{y}>$ stand here for unrounded non-front (central or back) vowels.

${ }^{15}$ Known cases include Austro-Bavarian as spoken in Amstetten (Traunmüller 1982) and Kensiu (Bishop 1996). 
- From Proto-Jê to Proto-Southern Jê (order $a \rightarrow b \rightarrow c$; ordering of $d$ and $e$ uncertain):

(a) lowering of ${ }^{*} a{ }^{*} a$;

(b) unrounding of ${ }^{*} \hat{o} /{ }^{*} 0 /{ }^{*} \tilde{o}>* \hat{\partial} / * a / * \tilde{\partial}$;

(c) lowering of near-close vowels: ${ }^{*} I>{ }^{*} \hat{e} ;{ }^{*} v>{ }^{*} \hat{o} ;{ }^{*} Y>{ }^{*} \hat{a}$ (accompanied by a push chain lowering: * $\left.\hat{\partial}>{ }^{*} a ;{ }^{*} a{ }^{*} a ;{ }^{*} a>{ }^{*} \tilde{a}\right)$;

(d) raising of $* \tilde{e}>{ }^{*} \tilde{i}$;

(e) fronting of ${ }^{*} \tilde{y}>{ }^{*} \tilde{i}$.

I do not reject other possible interpretations of the sound correspondences exposed above; the scenario detailed here is the most parsimonious I was able to find. Future studies of external correspondences will undoubtedly contribute to corroborate or reject at least some of my claims.

It must be noted that Proto-Southern Jê close-mid and open-mid oral vowels are further lowered $\left({ }^{*} \hat{\partial},{ }^{*} \hat{o},{ }^{*} \hat{e},{ }^{*} \partial,{ }^{*} 0,{ }^{*} e>{ }^{*} a,{ }^{*} 0,{ }^{*} e,{ }^{*} a,{ }^{*} a,{ }^{*} a\right.$, respectively) in syllables with nasal codas as a result of a synchronically active process. For space reasons, this process cannot be discussed here at length; the reader is referred to Nikulin (2015) for details.

Cognate sets that instantiate the aforementioned sound correspondences are provided below in (6-24).

(6) Proto-Jê ${ }^{*} a>$ Proto-Cerrado *a, Proto-Southern Jê * $\tilde{a}$

a. PJ *par 'foot' > PCerr *par, PSJ *pãn

b. PJ *pañ 'arm, branch'> PCerr *paj', PSJ *pã

c. PJ * $m b a$ 'to hear, to understand' > PCerr ${ }^{*} m b a{ }^{*} m b a-r$ ', PSJ ${ }^{*} m \tilde{a} /{ }^{*} m \tilde{a}-\eta$

d. PJ * $m b a$ 'liver' > PCerr ${ }^{*} m b a$, PSJ ${ }^{*} t \tilde{a}-m \tilde{a}$

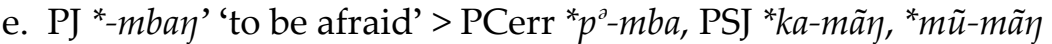

f. PJ *wa 'to walk' $>$ PNJ *wa 'to walk, to live', PSJ * $w \tilde{a}$ 'STAT' (aspect marker)

g. PJ ${ }^{*} r a$ 'to hit' > PNJ ${ }^{*} k u-r a /{ }^{*} k u-r a-\tilde{n}$, PSJ ${ }^{*} r \tilde{a} /{ }^{*} r \tilde{a}-\eta /{ }^{*} r \tilde{a}-n$

h. PJ *kapnja 'to chew' > PCerr *kapnjal*kapnja-r', PSJ *kajã

i. PJ “jara 'armpit; wing' > PNJ *jara 'wing', “jara-kre 'armpit', PSJ *jãrã (vb. *jãrã-n) 'armpit', * $\theta \tilde{a} r$ 'wing'

j. PJ *ja 'to stand.SG' > PCerr ${ }^{*} j a /{ }^{*} j a-m$ ', PSJ ${ }^{*} j \tilde{a} /{ }^{*} j \tilde{a}-\eta$

k. PJ ${ }^{*} j a$ 'to put vertically.SG' > PCerr ${ }^{*} \underline{j a} /{ }^{*} j \underline{a}-r$ ', PSJ ${ }^{*} \theta \tilde{a}\left(\mathrm{vb} .{ }^{*} \theta \tilde{a}-\eta\right)$ 'to be put vertically'

1. PJ * $\tilde{n} \tilde{i} j a$ 'nose' > PCerr * ${ }^{*} \tilde{i} j a-k r e$, PSJ *nĩja

m. PJ *jañ 'to eat.INTR, food'> PCJ ${ }^{*} c a j / /{ }^{*} c a$, PSJ ${ }^{*} j \tilde{a} /{ }^{*} j \tilde{a}-\eta /{ }^{*} j \tilde{a}-n\left(v b .{ }^{*} j \tilde{a}-n\right)$

n. PJ “kaj' 'basket' > PNJ *kac, PSJ *kãj (vb. *kãa)

o. PJ ${ }^{*} k r a$ 'offspring' > PCerr ${ }^{*} k r a$, PSJ ${ }^{*} k r a \tilde{a}\left(v b .{ }^{*} k r a \tilde{-} \eta\right)$

p. PJ *janda 'to send' > PCerr ${ }^{*} j a n d \underline{0} /{ }^{*} j a n d \underline{-}-r$ ', PSJ ${ }^{*} j \tilde{a} n \tilde{a} /{ }^{*} j a \tilde{a} \tilde{a}-\eta$

q. PJ *jarê ( *jarên) 'root'> PNJ *jarê, PSJ *järê (vb. * $\theta$-are-n) ${ }^{16}$

16 In examples (6q-s), one could be tempted to reconstruct PSJ ${ }^{*} a-$ rather than PSJ *- - - in unstressed (non-

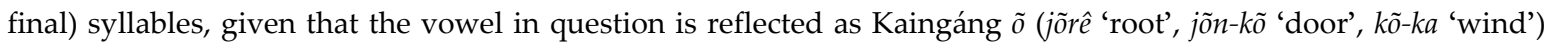
rather than $\tilde{e}$ (usually PSJ *a, * $\tilde{a}$ yield Kaingáng $\tilde{o}, \tilde{e}$, respectively). However, Laklãnõ data (jãrê 'root', $\partial$ - $\tilde{a} n-k a$ 'door') show that PSJ ${ }^{*} \tilde{a}$ must be reconstructed for these tokens. I propose to consider the following sound law: PSJ $* \tilde{a}>$ KGG $\tilde{e}$ in stressed (final) syllables as well as in unstressed (non-final) syllables if the stressed syllable also contains PSJ * $\tilde{a}>$ KGG $\tilde{e}$ (cf. PSJ *jãnã > KGG jẽnẽ 'to send'; PSJ *jãrã > KGG jẽre 'armpit'). Conversely, PSJ * $\tilde{a}>$ KGG õ in unstressed (non-final) syllables if the stressed syllable contains a different vowel (cf. PSJ *jãrê > KGG jõrê 'root'; PSJ *jãn-ka > KGG jõn-kõ 'door'; PSJ *jara > KGG jõra 'saliva'). Note that $\tilde{o}(\sim \tilde{a})$ and $\tilde{e}$ merged in all dialects other than Paraná Kaingáng and South-Eastern Kaingáng (Wiesemann 1978: 203-204); in some varieties, a new contrast between $\tilde{o}$ and $\tilde{e}$ emerged, whereby the use of $\tilde{o}$ is associated with round, compact objects and the use of $\tilde{e}$ is associated with long, diffuse objects (D’Angelis 2002). The Kaingáng forms given in this paper are from the Paraná dialect, where the etymological contrast is retained. 
r. PJ *jar-ko 'mouth'> PCerr *jad-kwa, PSJ *jãn-ka 'door'

s. PJ *kôk *ka-kôk 'wind'> PCerr *kôk, *ka-kôk, PSJ *kã-ka (vb. *kã-ka-n)

(7) Proto-Jê ${ }^{*}$ > Proto-Cerrado ${ }^{*} 0$, Proto-Southern Jê ${ }^{*} \tilde{a}$

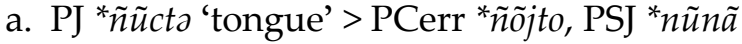

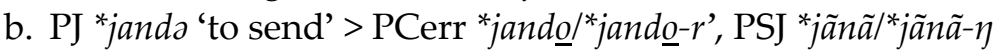

c. PJ *ndam 'eye' > PCerr ${ }^{*} n d o m$, PSJ ${ }^{*} k \partial-n \tilde{a}\left(\mathrm{vb} .{ }^{*} k \partial-n \tilde{a}-n\right)$ 'eye, fruit'

d. PJ *mbra 'ashes' > PCerr * mbro, PSJ * $m$ rã

e. PJ *ta 'to fly.SG' > PCerr ${ }^{*} t o /{ }^{*} t o p-r, \mathrm{PSJ}{ }^{*} t \tilde{a}\left(\mathrm{vb} .{ }^{*} t \tilde{a}-m\right)$

(8) Proto-Jê *â > Proto-Cerrado *â, Proto-Southern Jê *a

a. PJ * $m b \hat{\jmath}$ 'to grab' > PCerr * $m b \hat{\partial} /{ }^{*} m b \hat{\jmath}-r$ '; PSJ ${ }^{*} m b \partial /{ }^{*} m b a-\eta,{ }^{*} m b \partial-r$ (vb. ${ }^{*} m b a-n$ ) 'to hold, to carry (a short object)'

b. PJ *tât 'strong, hard' > PCerr *tât, PSJ *tar (vb. *tan)

c. PJ ${ }^{*} j \hat{j}, 3{ }^{*} c$ - $\hat{a}$ 'bitter' $>$ PCerr ${ }^{*} j \hat{\partial}$, PSJ ${ }^{*} \theta a\left(v b .{ }^{*} \theta a-\eta\right)$

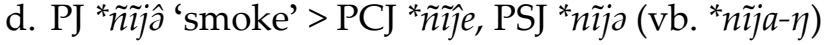

(9) Proto-Jê * $Y>$ Proto-Cerrado *a, Proto-Southern Jê *â

a. PJ *pry 'coal, ember' > PCerr *pra 'ember', PSJ *prô-n

b. PJ *jy 'urine' > PCerr *ja 'urine', PSJ * $j \hat{z}-\tilde{n}$ 'to urinate'

(10)Proto-Jê * $y>$ Proto-Cerrado * $y$, Proto-Southern Jê * $y$

a. PJ *pry ( *pryn) 'road' > PCerr *pry, PSJ * a-pry/*NP ja-pry

b. PJ *mbyt 'celestial body' > PCerr *mbyt 'sun', Ingain pyr 'moon'

c. PJ *mbyn 'tail' > PCerr *mbyn, PSJ * $m b y$

d. PJ *ty 'to die' > PCerr ${ }^{*} t y(r) /{ }^{*} t y k$, PSJ ${ }^{*} t y$ 'to die.STAT'

e. PJ *tyk 'black, dark' > PCerr *tyk 'black', PSJ *ku-ty (vb. *ku-ty- $\eta$ ) 'dark, night'

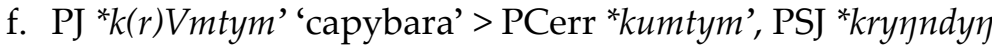

g. PJ * $c y$ 'to weave' $>$ PCerr ${ }^{*} c y /{ }^{*} c y p-r$ ', PSJ ${ }^{*} \theta y$

h. PJ ${ }^{*} c y m$ 'seed' > PCerr * ${ }^{*} y m$, PSJ ${ }^{*} \theta y\left(v b .{ }^{*} \theta y-n\right)$

i. PJ * $n \hat{\jmath} y$ 'bee, bumblebee' > PCerr ${ }^{*} n \hat{\jmath} y$, PSJ ${ }^{*} c y$

(11)Proto-Jê ${ }^{*} o>$ Proto-Cerrado * $w a$, Proto-Southern Jê * $a$

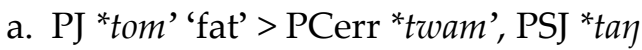

b. PJ *roñ 'celestial body' > PCerr *rwaj' 'moon', PSJ *ra 'sun'

c. PJ *jo 'tooth' > PCerr *jwa, PSJ *ja

d. PJ *kVjo 'salt' > PCerr *kVjwa, PSJ *kaja (vb. *kaja- $\eta)$ 'salty, sour'

e. PJ *jar-ko 'mouth'> PCerr *jad-kwa, PSJ *jãn-ka 'door'

f. PJ *kVñko 'sky' > PCerr *kajkwa, PSJ *kañka

g. PJ * $\eta g o \tilde{n}$ ' 'feather, hair' > PNJ * $\eta g w a ̂ \tilde{n}$ 'feather', PSJ * $\eta g a \tilde{n}$ 'animal hair'

(12)Proto-Jê ${ }^{*} \hat{o}>$ Proto-Cerrado * $\hat{o}$, Proto-Southern Jê *a

a. PJ *pôc 'to leave.PL'> PCerr *pôc, PSJ *pa (vb. *pa-n)

b. PJ *wô 'to untie' > PNJ * $b \hat{o} /{ }^{*} b \hat{o}-\tilde{n}, \mathrm{PSJ}{ }^{*} k \partial-w a /{ }^{*} k \partial-w a-\eta /{ }^{*} k \partial-w a-n$ 'to let loose, to untie'

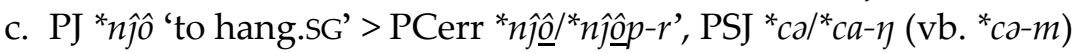

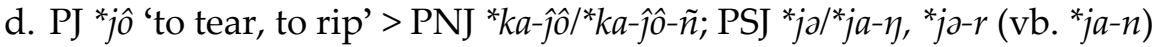

e. PJ *kôp 'fly, mosquito' > PCerr *kôp 'fly', PSJ *ka 'mosquito'

f. PJ *kôm 'tree, horn' > PCerr *kôm, PSJ *ka 'tree', * $n \tilde{\imath}-k a$ 'horn'

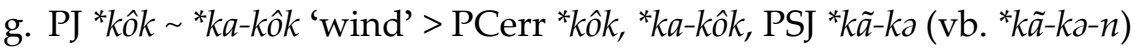

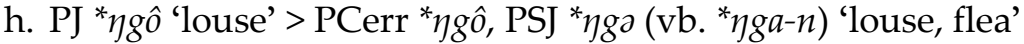


(13) Proto-Jê * $v>$ Proto-Cerrado * $u$, Proto-Southern Jê *ô
a. PJ *mbrom' 'ant' > PNJ *mbrũm, PSJ *mbroy-jy 'Argentine ant' (with nasal lowering)
b. PJ *tom' 'old'> PCerr *tum' 'old, tall'; PSJ *toy 'dry (of plants)' (with nasal lowering)
c. PJ *pondv 'bad or crooked' > PNJ *pundu 'bad', PSJ *pandô (vb. *pando- $n$ ) 'crooked'
d. PJ ${ }^{*} j v, 3{ }^{*} \mathrm{C}-v$ 'pus'> PCerr ${ }^{*} j u p-r, \mathrm{PSJ}{ }^{*} \theta \hat{o}\left(\mathrm{vb} .{ }^{*} \theta o-m\right)$

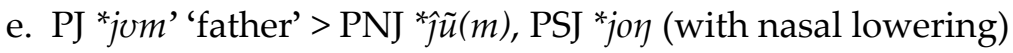
f. PJ * $k v$ 'to eat' $>$ PCerr ${ }^{*} k u /{ }^{*} k u-r$ ', PSJ ${ }^{*} k \hat{o}$ 'to eat, to use'

(14)Proto-Jê ${ }^{*} u>$ Proto-Cerrado * $u$, Proto-Southern Jê * $u$
a. PJ *tu 'to carry' > PCerr ${ }^{*} t u /{ }^{*} t u-r$ ', PSJ ${ }^{*} t u$ 'to carry on one's back, to wear'
b. PJ * $r u$ 'to transport liquid or to pour' > PNJ ${ }^{*} r u /{ }^{*} r u-\tilde{n}$ 'to spill, to pour', PSJ ${ }^{*} r u /{ }^{*} r u-\eta /$ ${ }^{*} r u-n$ 'to fetch water'
c. PJ *peju 'to hide' > PNJ *puĵu/ $p u \hat{\jmath} u-r$, PSJ *peju
d. PJ * $n \hat{\jmath} u$ 'to heat, to dry'> PNJ ${ }^{*} n \hat{\jmath} u /{ }^{*} n \hat{\jmath} u-\tilde{n}$ 'to dry in the sun'; PSJ ${ }^{*} C u-r\left(v b .{ }^{*} C u-n\right)$ 'to heat

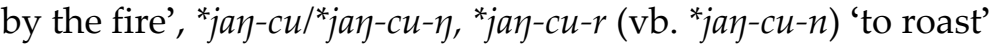

(15)Proto-Jê * $e>$ Proto-Cerrado *e, Proto-Southern Jê *e

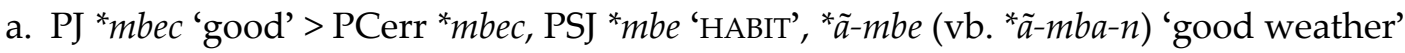
b. PJ *mbre > PNJ *mbre 'sibling-in-law', PSJ *ja-mbre 'cousin, son-in-law'
c. PJ ${ }^{*} r e$ 'to leave, to abandon' $>$ PCerr ${ }^{*} r e$ PSJ ${ }^{*} r e /{ }^{*} r a-\eta$
d. PJ *kre 'hole' > PCerr *kre, PSJ *kre
e. PJ * $\eta g r e$ 'egg' > PCerr * $y g r e$, PSJ * $y g r e$ 'egg, penis'

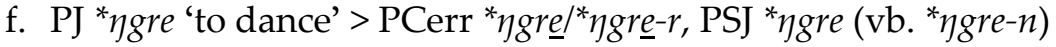

(16)Proto-Jê ${ }^{*} \hat{e}>$ Proto-Cerrado * $\hat{e}$, Proto-Southern Jê ${ }^{*} \hat{e}$
a. PJ *mbên 'liquid' > PCerr * mbên, PSJ *mbê (vb. * $m b e-n)$
b. PJ *jarê ( *jarên) 'root'> PNJ *jarê, PSJ *jarê (vb. *Oare-n)
c. PJ ${ }^{*}-r \hat{e}(C)$ 'to weed' $>$ PNJ ${ }^{*} k a-r \hat{e}{ }^{*} k a-r \hat{e}-r$, PSJ ${ }^{*} k u-r \hat{e} /{ }^{*} k u-r e-\eta$ 'to weed, to cut clean'

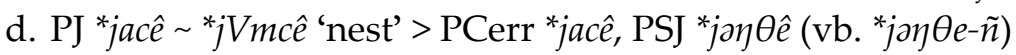
e. PJ " $n \hat{\jmath} \hat{e}$ 'to tickle' $>$ PNJ ${ }^{*} n \hat{\jmath} \hat{e} /{ }^{*} n \hat{\jmath} \hat{e}-r$ ', PSJ ${ }^{*} k u-\left.c \hat{e}\right|^{*} k u-c e-\eta$
f. PJ “jê ( *jên) 'thread, knot, cloth' > PNJ * $\hat{\jmath} \hat{e}, \mathrm{PSJ} v \mathrm{vb} .{ }^{*} \theta e-n$ 'to spin thread'
g. PJ “kêt 'NEG'> PNJ *kêt, PSJ *kêr 'ADVT'

(17)Proto-Jê ${ }^{*} I>$ Proto-Cerrado *ja, Proto-Southern Jê * $\hat{e}$
a. PJ *mbın' 'husband' > PNJ *mbjên, PSJ *mben (with nasal lowering)
b. PJ *kIj 'pit' > PNJ *kî 'earth oven', PSJ *kêj 'tomb'
c. PJ ${ }^{*} k_{I} \sim{ }^{*} k_{I j} \sim{ }^{*} k I j$ ' 'to split' > PNJ ${ }^{*} k o-k \hat{\imath} /{ }^{*} k o-k j \hat{e}-r$, PSJ vb. ${ }^{*} k e-\tilde{n}$
d. PJ *krIñ 'thigh' > PCerr ${ }^{*} k(r) j a j$ ', PSJ ${ }^{*} k r e \hat{e}$
e. PJ “ $\eta g I$ 'to enter.PL' > PCerr ${ }^{*} a-\eta g j a /{ }^{*} \eta g j a-c$, PSJ ${ }^{*} \eta g \hat{e}\left(v b .{ }^{*} \eta g \hat{e}-m\right)$

(18)Proto-Jê ${ }^{*} i>$ Proto-Cerrado ${ }^{*} i$, Proto-Southern Jê ${ }^{*} i$
a. PJ *peti 'to dream' > PNJ *pyti/*pyti-r, PSJ ${ }^{*}$ peti/*peti- $\eta$
b. PJ *ji 'to lay.SG'> PCerr ${ }^{*} j i{ }^{*} j i-r$ ', PSJ ${ }^{*} j i /{ }^{*} j i-\eta$

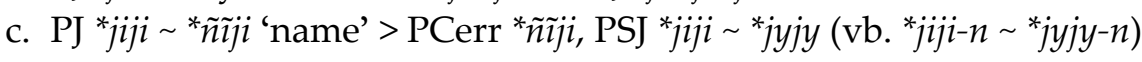

(19)Proto-Jê *̃̃ > Proto-Cerrado *ã, Proto-Southern Jê *ã
a. PJ *prãm' 'hungry, to want' > PCerr *prãm', PSJ *prã 'hungry, year'
b. PJ *tã 'INSTR' > PCerr * $t \tilde{a}$, PSJ *tã 'ERG, INSTR'
c. PJ *nã 'mother' > PCerr ${ }^{*} n \tilde{a}$, PSJ *nã
d. PJ * $\eta \tilde{\partial}(C)$ 'to push against, to grind'> PNJ ${ }^{*} \eta \tilde{\partial} /{ }^{*} \eta \tilde{\partial}-\tilde{n}$, PSJ ${ }^{*} \eta \tilde{\partial} /{ }^{*} \eta \tilde{\partial}-\eta$ 
(20)Proto-Jê * $\tilde{y}>$ Proto-Cerrado *̃ $\left({ }^{*} \tilde{y}\right.$ after palatals), Proto-Southern Jê ${ }^{*} \tilde{\imath}$
a. PJ * $\tilde{n} \tilde{y}$ 'to sit.SG' > PCerr ${ }^{*} \tilde{n} \tilde{y} /{ }^{*} \tilde{n} \tilde{y} p-r$, PSJ ${ }^{*} n \tilde{\imath}\left(v b .{ }^{*} n \tilde{\imath}-m\right)$

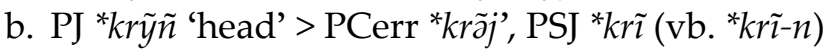

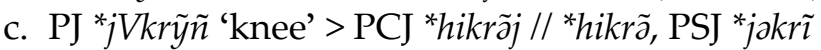

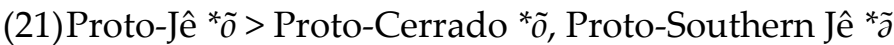

a. PJ * $p \tilde{o}$ 'to rub, to clean' $>$ PNJ * $p \tilde{o} /{ }^{*} p \tilde{o}-\tilde{n}$, PSJ ${ }^{*} p \tilde{\partial} /{ }^{*} p \tilde{z}-\eta$ 'to clean a field'

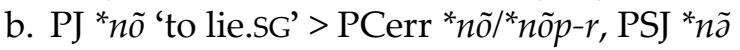

(22)Proto-Jê * $\tilde{u}>$ Proto-Cerrado *̃o, Proto-Southern Jê * $\tilde{u}$
a. PJ *prũ 'wife' > PCerr *prõ, PSJ *prũ (vb. *prũ- $\eta$ )
b. PJ *mũ 'to go.PL' > PCerr ${ }^{*} m \tilde{o} /{ }^{*} m \tilde{o}-r$ ', PSJ ${ }^{*} m \tilde{u}\left(\mathrm{vb} .{ }^{*} m \tilde{u}-n\right)$
c. PJ ${ }^{*} t \tilde{u}$ 'NEG' $>$ PCJ ${ }^{*} t \tilde{o}, \mathrm{PSJ}{ }^{*} t \tilde{u} /{ }^{*} t \tilde{u}-\eta\left(\mathrm{vb} .{ }^{*} t \tilde{u}-\eta\right)$
d. PJ * $\tilde{u} t /{ }^{*} \tilde{n} \tilde{u} t$ 'to sleep' > PCerr ${ }^{*} \eta \tilde{o} t /{ }^{*} \tilde{n} \tilde{o} t, \mathrm{PSJ}{ }^{*} n \tilde{u} r$

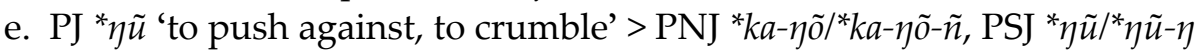

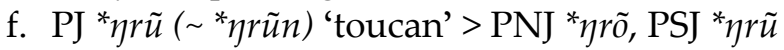

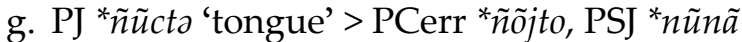
h. PJ * $\tilde{n} \tilde{u} m j \hat{e}(C)$ 'female breast' > PNJ * $\tilde{n} \tilde{m} m \hat{\jmath} \hat{e}, \mathrm{PSJ}{ }^{*} n \tilde{u} \eta j \hat{e}$ 'female breast, milk'

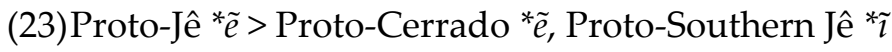

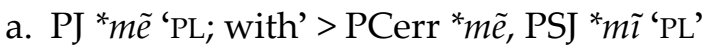
b. PJ *te 'to go.SG' > PCerr ${ }^{*} t \tilde{e} /{ }^{*} t \tilde{e} m^{\prime}$, PSJ ${ }^{*} t \tilde{\imath} /{ }^{*} t \tilde{\imath}-\eta\left(\mathrm{vb} .{ }^{*} t \tilde{\imath}-n\right)$
c. PJ ${ }^{*} r \tilde{e}$ 'to throw.PL' $>$ PNJ ${ }^{*} r \tilde{e} /{ }^{*} r \tilde{e}-\tilde{n}$, PSJ ${ }^{*} r \tilde{l} /{ }^{*} r \tilde{l}-\eta /{ }^{*} r \tilde{l}-\tilde{n}$

(24)Proto-Jê ${ }^{*} \tilde{\imath}>$ Proto-Cerrado *ĩ, Proto-Southern Jê *ĩ
a. PJ “pĩm 'tree, wood' > PCerr *pim, PSJ * $p \tilde{\imath}$ 'fire, firewood'
b. PJ ${ }^{*}-\tilde{i}(C)$ 'bandage, sling' $>$ PNJ ${ }^{*} j a-c \tilde{\imath},{ }^{*} k a-c \tilde{\imath}$, PSJ ${ }^{*}-\theta \tilde{\imath}(v b .-\theta \tilde{\imath}-n)$
c. PJ “ $\tilde{n} \tilde{\imath}$ 'meat' > PCerr ${ }^{*} \tilde{n} \tilde{l}$, PSJ ${ }^{*} n \tilde{\imath}$
d. PJ * $\tilde{n} \tilde{\imath}(m)$ - 'hand (in compounds)' > PCerr ${ }^{*} \tilde{n} \tilde{\imath}(m)-k r a$ 'hand', PSJ ${ }^{*} n \tilde{\imath}-$ (as in ${ }^{*} n \tilde{\imath}-p \hat{e}$ 'to wash hands')

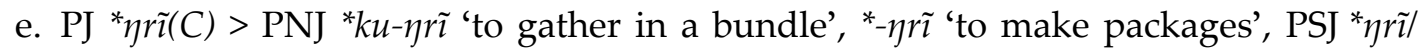 * $\eta r \tilde{i}-\eta$, * $\eta r \tilde{i}-r(v b . ~ * \eta r \tilde{l}-n)$ 'wrap'

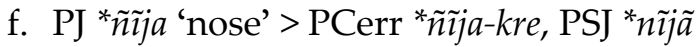

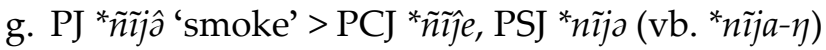

In non-final (unstressed) syllables, divergent correspondences may be observed. One such correspondence involves the PCerr formative that is reflected in PNJ as ${ }^{*} p y-{ }^{*} p u$ - word-initially (harmonizing in roundness with the nucleus of the root, cf. Salanova 2011a: 66) or as ${ }^{*}-p$ word-medially, and in PCJ as * pi-. In some stems, this formative corresponds to PSJ *pa- (25ab); in others, it corresponds to PSJ ${ }^{*} p e-(25 c-d)$, thus evidencing a merger that would have occurred historically in PCerr. I assume that PSJ displays here the same reflexes of PJ vowels that are found in stressed syllables, whereas in the history of PCerr the unstressed vowels ${ }^{*} O$ and ${ }^{*} e$ were reduced to zero (probably via ${ }^{*} u{ }^{*} i$, since high vowels are more likely to be syncopated). The consonant clusters created by this development were subsequently undone via different processes in PNJ (resyllabification if available, epenthesis of ${ }^{*} y /{ }^{*} u$ elsewhere) and in PCJ (epenthesis of ${ }^{*} i$ ).

(25) Proto-Jê ${ }^{*} 0$, $e>$ PCerr zero in unstressed syllables

a. PJ *pondv 'bad or crooked' > PCerr ${ }^{*} p^{\curvearrowright} n d u$ (PNJ *pundu 'bad'), PSJ *pandô (vb. *pando- $n$ ) 'crooked'

b. PJ *potu 'horsefly' > PCerr * $p^{\curvearrowright} t u$ (PNJ *pucu, PCJ *pidu), PSJ *patu 


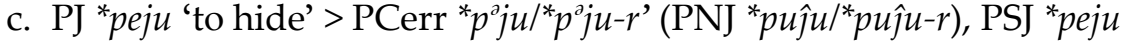 \\ d. PJ *peti 'to dream'> PCerr ${ }^{*} p^{a} t i{ }^{*} p^{a} t i-r$ ' (PNJ $\left.{ }^{*} p y t i /{ }^{*} p y t i-r\right)$, PSJ ${ }^{*} p e t i /{ }^{*} p e t i-\eta$}

There appear to be no counterexamples to the development exemplified in (25): the usual reflexes of PJ ${ }^{*} 0$ and ${ }^{*} e$ (PCerr ${ }^{*} w a>$ PNJ ${ }^{*} w a,{ }^{*} w \hat{a}, \mathrm{PCJ}{ }^{*} w a$; PCerr $\left.{ }^{*} e>\mathrm{PNJ}{ }^{*} e, \mathrm{PCJ}{ }^{*} \hat{e}\right)$ do not occur in prefixes or in the unstressed syllables of underived stems in the Cerrado languages.

Only one well-known cognate set violates the regularities outlined in this section: as shown in (26), it features a correspondence between PCerr ${ }^{*} \hat{o}$ and PSJ ${ }^{*} e$. Judging by the external cognates, such as Maxakalí -cyc (orthographically $<-x u x>$ ) or Krenák jat, PCerr is conservative here: the correspondence between Maxakalí $y$, Krenák $\partial$, and PCerr ${ }^{*} \hat{o}$ is well-attested (these vowels usually correspond to PSJ ${ }^{*}$ ), whereas PSJ ${ }^{*} e$ usually has entirely different correspondences: Maxakalí e, Krenák i, PCerr *e (Nikulin \& Silva, forthc.).

(26) An exceptional correspondence between PCerr ${ }^{*} \hat{o}$ and PSJ ${ }^{*} e$

PJ *côj 'leaf' > PCerr * ${ }^{*} \hat{j} j$ ', PSJ ${ }^{*} \theta e j\left(\right.$ vb. $\left.{ }^{*} \theta e-\tilde{n}\right)$

I hypothesize that PSJ ${ }^{*} e$ in ${ }^{*} \theta e j$ is a result of fronting of pre-PSJ ${ }^{*}$ (the regular outcome of PJ $\left.{ }^{*} \hat{o}\right)$ before a palatal coda. The development ${ }^{*}-\partial j>{ }^{*}-e j$ may have been regular: the rhyme ${ }^{*}-\partial j$ does not occur abundantly in Proto-Southern Jê lexicon, the only known example being *waj 'dawn' without a known external etymology. However, in the absence of supporting examples the hypothesis in question remains rather speculative.

\subsection{Codas}

The correspondences between the codas of PCerr and PSJ, as well as the respective Proto-Jê reconstructions are presented in Table 7 below. I remind the reader that the apostrophe stands for a suppressed echo vowel (word-finally). For Southern Jê, in addition to the codas found in underived stems, I provide information regarding the allomorphy of the verbalizer/causative suffix. Four codas $\left({ }^{*} \mathrm{p}^{\prime},{ }^{*} \mathrm{t}^{\prime},{ }^{*} \mathrm{c}^{\prime},{ }^{*} \mathrm{k}\right.$ '/) are reconstructed only for the underlying level of PJ.

Cognate sets that instantiate the aforementioned sound correspondences (excepts those going back to PJ underlying codas, for which see below) are provided below in (27-40).

(27)Proto-Jê * $p>$ Proto-Cerrado * $p$, Proto-Southern Jê * $\varnothing$

a. PJ *kôp 'fly, mosquito' > PCerr *kôp 'fly', PSJ *ka 'mosquito'

(28)Proto-Jê ${ }^{*} t>$ Proto-Cerrado *t, Proto-Southern Jê ${ }^{*} r\left(v b .{ }^{*}-n\right)$

a. PJ *mbyt $>$ PCerr *mbyt 'sun', Ingain pyr 'moon'

b. PJ *tât 'strong, hard' > PCerr *tât, PSJ *tar (vb. *tan)

c. PJ " rĩt 'to look' > PCerr ${ }^{*}$ ritt, PSJ ${ }^{*}$ rĩr

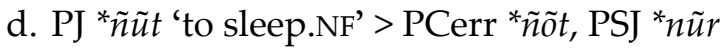

e. PJ *kẽt 'stone' > PCerr ${ }^{*} k e \tilde{t}$, Ingain $k \tilde{e} r$

f. PJ *krot 'chin, beak'> PCerr *krwat 'beak', Ingain jat-krar 'chin'

(29) Proto-Jê ${ }^{*} c>$ Proto-Cerrado ${ }^{*} c$, Proto-Southern Jê ${ }^{*} \emptyset\left(\right.$ vb. $\left.{ }^{*}-n\right)$

a. PJ *pôc 'to leave.PL'> PCerr *pôc, PSJ *pa (vb. *pa-n)

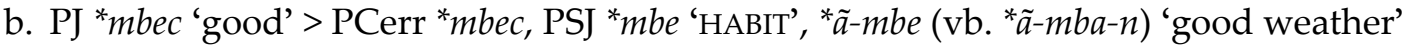

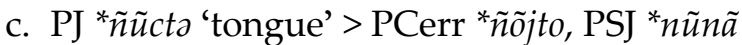

(30) Proto-Jê ${ }^{*} k>$ Proto-Cerrado * $k$, Proto-Southern Jê ${ }^{*} \varnothing\left(\right.$ vb. $\left.{ }^{*}-n\right)$ or ${ }^{*} j\left(\right.$ after $\left.{ }^{*} \hat{e}\right)$

a. PJ *tyk 'black, dark' > PCerr *tyk 'black', PSJ *ku-ty (irreg. vb. *ku-ty- $\eta$ ) 'dark, night'

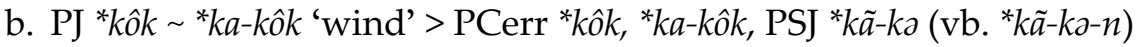


Table 7. Proto-Jê codas and their reflexes in Proto-Cerrado and Proto-Southern Jê.

\begin{tabular}{|c|c|c|c|}
\hline PJ & PCerr & PSJ & PSJ (vb.) \\
\hline \multicolumn{4}{|c|}{ followed by echo vowels } \\
\hline${ }^{*} \mathrm{p}$ & ${ }^{*} \mathrm{p}$ & $* \varnothing$ & \multirow{7}{*}{${ }^{*}-\mathrm{n}$} \\
\hline$*_{t}$ & $*_{t}$ & ${ }^{*} \mathrm{r}$ & \\
\hline$*^{*} \mathrm{C}$ & ${ }^{*} \mathrm{C}$ & $* \varnothing$ & \\
\hline${ }^{*} \mathrm{k}$ & ${ }^{*} \mathrm{k}$ & $* \emptyset,{ }^{*} j^{+}$ & \\
\hline${ }^{*} \mathrm{~m}$ & ${ }^{*} \mathrm{~m}$ & \multirow{3}{*}{$* \varnothing$} & \\
\hline${ }^{*} \mathrm{n}$ & ${ }^{*} \mathrm{n}$ & & \\
\hline${ }^{*} \tilde{\mathrm{n}}$ & *j' & & \\
\hline
\end{tabular}

\begin{tabular}{|l|l|l|l|}
\hline${ }^{*} \mathrm{r}$ & ${ }^{*} \mathrm{r}$ & ${ }^{*} \mathrm{n}$ & ${ }^{*}-\mathrm{n}$ \\
\hline${ }^{*} \mathrm{j}$ & ${ }^{* j}$ & ${ }^{*} \mathrm{j}$ & ${ }^{*}-\tilde{n}$ \\
\hline
\end{tabular}

\begin{tabular}{|c|c|c|c|c|}
\hline & PJ & PCerr & PSJ & PSJ (vb.) \\
\hline \multicolumn{5}{|c|}{ no echo vowels } \\
\hline \multirow{4}{*}{$* \varnothing$} & $* / p^{\prime} /$ & ${ }^{*} \emptyset,{ }^{*} \mathrm{p}^{\ddagger}$ & \multirow{4}{*}{${ }^{*} \varnothing$} & $*-m$ \\
\hline & $* / t^{\prime} /$ & \multirow{3}{*}{${ }^{*} \varnothing$} & & $*-n$ \\
\hline & $* / c^{\prime} /$ & & & $*-\tilde{n}$ \\
\hline & $* / k^{\prime} /$ & & & $*-n$ \\
\hline & 'm' & ${ }^{*} \mathrm{~m}$ ' & ${ }^{*} n$ & ${ }^{*}-n$ \\
\hline & *n’ & *n' & ${ }^{*} \mathrm{n}$ & $*-n$ \\
\hline & * $\tilde{n} '$ & * $\tilde{n}$ ' & ${ }^{*} \tilde{\mathrm{n}}$ & $*-\tilde{n}$ \\
\hline & * $n^{\prime}$ & (?) & ${ }^{*} y$ & $*-\eta$ \\
\hline & ${ }^{*} \mathrm{r}$ & *d & ${ }^{*} \mathrm{n}$ & $*-n$ \\
\hline & *j' & ${ }^{*} \mathrm{C}$ & $*_{j}$ & *-ñ \\
\hline
\end{tabular}

$\dagger$ after ${ }^{*} \hat{e}^{17}$ (no vb. attested, but probably $\left.{ }^{*} \tilde{n}\right) ; \ddagger$ in non-finite forms preceding the suffix ${ }^{*}-r$
c. PJ “pêk 'to fart.NF' > PNJ *pê- $k$, PSJ *pêj (but PCJ *pi-ri)
d. PJ *kujêk 'vein'> PNJ *kuĵ̂k, PSJ *kujêj

(31)Proto-Jê ${ }^{*} m>$ Proto-Cerrado ${ }^{*} m$, Proto-Southern Jê ${ }^{*} \emptyset\left(\mathrm{vb} .{ }^{*}-n\right)$
a. PJ *pĩm 'tree, wood' > PCerr *pim, PSJ *pi 'fire, firewood'

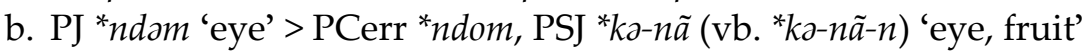
c. PJ ${ }^{*} c y m$ 'seed' > PCerr ${ }^{*} c y m$, PSJ ${ }^{*} \theta y\left(v b .{ }^{*} \theta y-n\right)$
d. PJ *kôm 'tree, horn' > PCerr *kôm, PSJ *ka 'tree', *nĩ-ka 'horn'

(32)Proto-Jê ${ }^{*} n>$ Proto-Cerrado * $m$, Proto-Southern Jê ${ }^{*} \emptyset$ (vb. * $\left.{ }^{*} n\right)$

a. PJ *mbyn 'tail' > PCerr * $m b y n$, PSJ * $m b y$

b. PJ *mbên 'liquid' > PCerr *mbên, PSJ *mbê (vb. *mbe-n)

(33)Proto-Jê * $\tilde{n}>$ Proto-Cerrado *j', Proto-Southern Jê ${ }^{*} \emptyset\left(\right.$ vb. $\left.{ }^{*}-n\right)$
a. PJ *pañ 'arm, branch'> PCerr *paj', PSJ *pa
b. PJ ${ }^{*} j a \tilde{n}$ 'to eat.INTR, food'> PCJ ${ }^{*} \mathrm{caj} / /{ }^{*} \mathrm{Ca}, \mathrm{PSJ}{ }^{*} j \tilde{a} /{ }^{*} j \tilde{a}-\eta /{ }^{*} j \tilde{a}-n\left(\mathrm{vb} .{ }^{*} j \tilde{a}-n\right)$

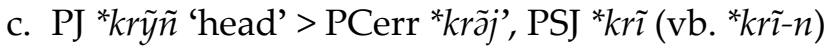
d. PJ *kriñ 'thigh' > PCerr *k(r)jaj', PSJ *krê

(34)Proto-Jê ${ }^{*} r>$ Proto-Cerrado * $r$, Proto-Southern Jê ${ }^{*} n$
a. PJ *par 'foot' > PCerr *par, PSJ *pãn

${ }^{17}$ I thank Mário André Coelho da Silva for pointing out the possibility that PJ *êk > PSJ *-êj could be a regular sound change. 
(35)Proto-Jê *j> Proto-Cerrado *j', Proto-Southern Jê *j (vb. *-ñ)

a. PJ *côj 'leaf'> PCerr * ${ }^{*} \hat{j}$ ', PSJ ${ }^{*} \theta e j\left(\mathrm{vb} .{ }^{*} \theta e-\tilde{n}\right)$

(36)Proto-Jê * $m$ '> Proto-Cerrado * $m$ ', Proto-Southern Jê * $\eta$

a. PJ *prãm' 'hungry' > PCerr *prãm', PSJ *prã $\eta$ 'hungry; year'

b. PJ *mbrom' 'ant' > PNJ *mbrũm, PSJ *mbroy-jy 'Argentine ant'

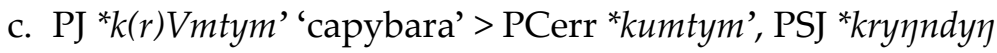

d. PJ *tom' 'fat' > PCerr *twam', PSJ *tay

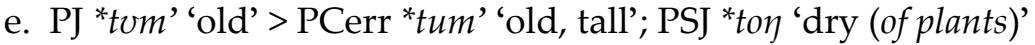

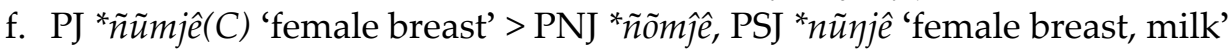

(37)Proto-Jê * $n$ ' > Proto-Cerrado * $n$ ', Proto-Southern Jê * $n$

a. PJ *mbin' 'husband'> PNJ *mbjên, PSJ *mben

(38)Proto-Jê * $\tilde{n}$ ' > Proto-Cerrado * $\tilde{n}$ ', Proto-Southern Jê * $\tilde{n}$

a. PJ * $\eta g o \tilde{n}$ ' 'feather, hair' > PNJ * $\eta g w a ̂ \tilde{n}$ 'feather', PSJ * $\eta g a \tilde{n}$ 'animal hair'

(39)Proto-Jê * $r$ ' > Proto-Cerrado *d, Proto-Southern Jê * $n$

a. PJ *jar-ko 'mouth'> PCerr *jad-kwa, PSJ *jãn-ka 'door'

(40)Proto-Jê *j'> Proto-Cerrado *j', Proto-Southern Jê ${ }^{*} j\left(\right.$ vb. $\left.{ }^{*}-\tilde{n}\right)$

a. PJ *kaj' 'basket' > PNJ ${ }^{*} k a c$, PSJ ${ }^{*} k \tilde{a} j\left(v b .{ }^{*} k \tilde{a} \tilde{n}\right)$

The reconstruction of PJ ' $\eta$ ' is supported by only one PSJ token, PSJ “ $m \tilde{\partial} \eta$ 'honey bee', which lacks known cognates in Cerrado languages but corresponds externally to Maxakalí pyk (orthographically $\langle p u k>$ ) and Krenák poๆ. As Nikulin and Silva (forthc.) show, the point of articulation of Proto-Jê codas systematically matches that of Maxakalí, and the manner of articulation of Proto-Jê codas systematically matches that of Krenák.

My reconstruction of Proto-Jê underlying codas (/* $\mathrm{p}^{\prime},{ }^{*} \mathrm{t}^{\prime},{ }^{*} \mathrm{c}^{\prime},{ }^{*} \mathrm{k}$ '/) is crucially based on morphophonological evidence from Central and Southern Jê and is corroborated by external comparison with Maxakalí and Krenák (Nikulin \& Silva, forthc.). No contemporary Jê language shows any reflex of these codas in underived environments, which probably means that they surfaced as zero in underived Proto-Jê words. In Central Jê, only PJ /*p'/ left any trace: in non-finite forms of verbs, it combined with the non-finite suffix ${ }^{*}-r$, yielding PCJ ${ }^{*} b-r V$. In Southern Jê, the quality of the underlying codas is made manifest in derived denominal verbs or causatives, where underlying /-n/ acquires the point of articulation of the underlying coda consonant (see Cavalcante 1987: 51-52 for a synchronic analysis for São Paulo Kaingáng). Examples are given in (41-44).

(41)Proto-Jê $* / p^{\prime} />$ Proto-Cerrado ${ }^{*} \varnothing\left(\mathrm{NF}^{*}-p-r\right)$, Proto-Southern Jê ${ }^{*} \emptyset\left(\mathrm{vb} .{ }^{*}-m\right)$

a. PJ *ta*/təp'/ 'to fly.SG' > PCerr ${ }^{*}$ to/*top-r, PSJ ${ }^{*} t \tilde{a}\left(\mathrm{vb} .{ }^{*} t \tilde{a}-m\right)$

cf. Maxakalí /tup-a/, IRR/tup/ 'id.'

b. PJ *nõ *nõp'/ 'to lie.SG' > PCerr *nö/*nõp-r, PSJ *ñ̃

cf. Maxakalí /nũp/ 'to lie.PL'

c. PJ *cy*/cyp'/ 'to weave' > PCerr *cy/*cyp-r', PSJ * $\theta y$

cf. Maxakalí /cap/ 'id.'

d. PJ ${ }^{*} c \hat{o}{ }^{*} / \mathrm{côp}$ '/ 'to eat soft food, to suck' > PNJ ${ }^{*} c \hat{o} /{ }^{*} c \hat{o}-r$, PSJ ${ }^{*} p e-\theta a\left(\mathrm{vb} .{ }^{*} p e-\theta a-m\right)$

'to suck breast'

cf. Maxakalí /cyp/ 'to suck, to lick'

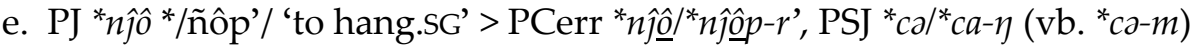

cf. Maxakalí /cyp/ 'id.' 
f. PJ * $\tilde{y} \tilde{y}{ }^{*} / \mathrm{jy} \mathrm{p}$ '/ 'to sit.SG' > PCerr ${ }^{*} \tilde{n} \tilde{y} /{ }^{*} \tilde{n} \tilde{y} p-r$, PSJ ${ }^{*} n \tilde{\imath}\left(\right.$ vb. $\left.{ }^{*} n \tilde{\imath}-m\right)$

cf. Maxakalí /ñỹp/ 'id.'

g. PJ *jv*/jop'/ 'pus'> PCerr ${ }^{*}$ jup $-r$, PSJ ${ }^{*} \theta \hat{o}\left(\mathrm{vb} .{ }^{*} \theta o-m\right)$

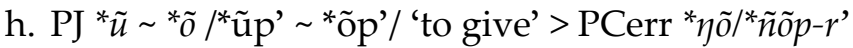

cf. Maxakalí /hũp/ 'id.'

(42)Proto-Jê ${ }^{*} / \mathrm{t}^{\prime} />$ Proto-Cerrado ${ }^{*} \emptyset$, Proto-Southern Jê ${ }^{*} \emptyset\left(\mathrm{vb} .{ }^{*}-n\right)$

a. PJ *jarê $(\sim$ *jarên $) *$ /jarêt' jarên/ 'root' > PNJ *jarê, PSJ *jãrê (vb. * $\theta$-are-n)

cf. Maxakalí /ñ̃̃p-catit/ 'id.'

b. PJ *jê ( *jên $)^{*} /$ jêt' jên/ 'thread, knot, cloth' > PNJ *jêe, PSJ vb. * $\theta e-n$ 'to spin thread' cf. Maxakalí /cit/ 'id.'

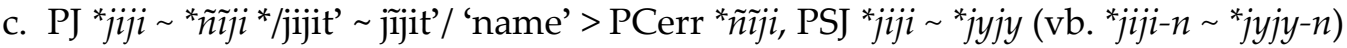
cf. Maxakalí /-cit/ 'id.'

d. PJ ${ }^{*} k o{ }^{*} / \operatorname{kot}^{\prime} /$ 'to dig' $>$ PNJ ${ }^{*} k w \hat{a} /{ }^{*} k w \hat{a}-\tilde{n}$, PSJ ${ }^{*} k a /{ }^{*} k a-\eta\left(v b .{ }^{*} k a-n\right)$ cf. Maxakalí /kut/ 'id.'

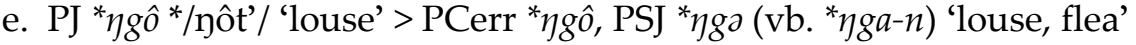
cf. Maxakalí /kyt/ 'id.'

(43)Proto-Jê $* / c^{\prime} />$ Proto-Cerrado * $\varnothing$, Proto-Southern Jê ${ }^{*} \varnothing\left(v b .{ }^{*}-\tilde{n}\right)$

a. PJ *pry */pryc'/ 'coal, ember' > PCerr *pra 'ember', PSJ * $p r \hat{a}-\tilde{n}^{18}$

b. PJ *jY ${ }^{*} / \mathrm{jyc}$ / 'urine' > PCerr *ja 'urine', PSJ vb. ${ }^{*} j \hat{\partial}-\tilde{n}$ 'to urinate' cf. Maxakalí /cyc/ 'id.'

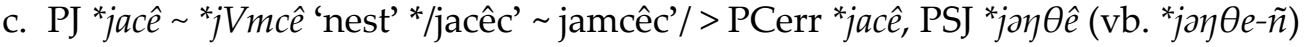

(44)Proto-Jê ${ }^{*} / \mathrm{k}^{\prime} />$ Proto-Cerrado ${ }^{*} \emptyset$, Proto-Southern Jê ${ }^{*} \emptyset\left(\mathrm{vb} .{ }^{*}-n\right)$

a. PJ ${ }^{*} t \tilde{u}{ }^{*} /$ tũk' / 'NEG'> PCJ ${ }^{*} t \tilde{o}$, PSJ ${ }^{*} t \tilde{u} /{ }^{*} t \tilde{u}-\eta\left(v b .{ }^{*} t \tilde{u}-\eta\right)$

cf. Maxakalí /nũk/ 'to end, to run out'

b. PJ ${ }^{*} k r a * / k r a k ' /$ 'offspring' > PCerr ${ }^{*} k r a$, PSJ ${ }^{*} k r a \tilde{a}\left(\mathrm{vb} .{ }^{*} k r \tilde{a}-\eta\right)$

cf. Maxakalí /ktuk/ 'id.'

I do not represent these underlying codas in my reconstructions throughout this paper; the examples (41-44) above include almost every cognate set for which the presence of an underlying coda may be established with any certainty.

\section{Jê etymologies}

In this section, we present the most reliable cognate sets identified so far. The data are sorted by the onset of the final (stressed) syllable, then by its nucleus, then by its coda, then by any preceding material, following the order $/ p, p r, m, m r, w, t, n, d, r, c, \tilde{n}, j, k, k r, y, n r, a, ~ \partial, ~ \tilde{\partial}, \hat{\partial}, x$, y, $\tilde{y}, \mathrm{o}, \tilde{\mathrm{o}}, \hat{\mathrm{o}}, \mathrm{v}, \mathrm{u}, \tilde{\mathrm{u}}, \mathrm{e}, \tilde{\mathrm{e}}, \hat{\mathrm{e}}, \mathrm{I}, \mathrm{i}, \tilde{\mathrm{i}} /$. I list first the etymologies that have reflexes both in Cerrado and in Southern Jê languages, and then proceed to the reconstructions that are based only on Cerrado or Southern Jê reflexes (as well as their external cognates elsewhere in Macro-Jê or Tupian $\left.{ }^{19}\right)$, respectively.

\footnotetext{
${ }^{18}$ It is unclear why the PSJ reflex looks like a verbalized form.

${ }^{19}$ Although I do not currently regard the relation between Macro-Jê and Tupian as conclusively proven, I still find the hypothesis quite promising. Some novel lexical evidence may be found in Nikulin \& Silva forthc. (as well as in this section). The Proto-Tupian reconstructions are my own and follow the principles outlined by Carvalho and Nikulin (in prep.).
} 
For reasons of space, I do not provide the data of individual Northern Jê languages, limiting myself to Proto-Northern Jê reconstructions. Detailed information on the reflexes of ProtoNorthern Jê items in individual Northern Jê languages may be found in Nikulin \& Salanova (forthc.). Ingain and Southern Kayapó data are only given for cognate sets that lack known reflexes in Kaingáng/Laklãnõ and Panará, respectively.

\section{Reflexes in both branches}

PJ *par 'foot':

PCerr *par > PNJ *par; PNR pa:; PCJ *para // *pa:ra (XAV para // pa:ra, XER pra)

PSJ *pãn > KGG pẽn; LKL pãn

PJ *pañ 'arm, branch':

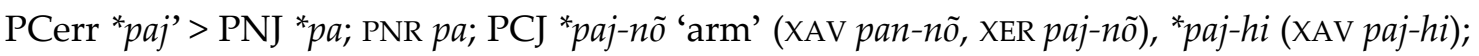

(?) PCJ *pa-krata // *pa-kra:da 'root' (XER pa-krta // pa-krda), (?) *wêdê-pa 'root' (XAV wêdê$p a$, XER $w d \hat{e}-p a)$

PSJ * $p \tilde{a}>$ KGG $p \tilde{e} ;$ LKL $p \tilde{a}$

PJ *pĩm 'tree, wood, firewood':

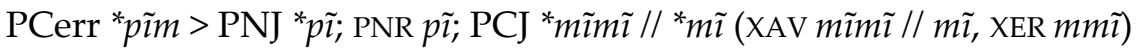

PSJ * $p \tilde{\imath}$ 'fire, firewood'> KGG $p \tilde{\imath}$; LKL $p \tilde{e}$

PJ * $p \tilde{o}$ 'to rub, to clean':

PCerr ${ }^{*} p \tilde{o} /{ }^{*} p \tilde{o}-\tilde{n}$ '> PNJ ${ }^{*} p \tilde{o} / * p \tilde{o}-\tilde{n}$

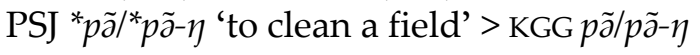

PJ *pôc 'to leave.PL':

PCerr ${ }^{*} p \hat{o} c>$ PNJ ${ }^{*} t o=p o \hat{j} j{ }^{*} t o=p o ̂ c$ 'to extract.PL'; PCJ *pu/*pu-ci 'to leave.DU' (XAV pu-ci, XER $\mathrm{pu} / \mathrm{pu}-\mathrm{s}(\mathrm{i}))$

PSJ *pa (vb. *pa-n)> KGG $p a(\mathrm{vb} . p a-n) ;$ LKL $p o$

$\mathrm{PJ} * p \hat{e} /{ }^{*} p \hat{e}-k$ 'to fart':

PCerr ${ }^{*} p \hat{e} /{ }^{*} p \hat{e}-k>\mathrm{PNJ}{ }^{*} i j-p \hat{e}{ }^{*} p \hat{e}-k ; \mathrm{PCJ}{ }^{*} p i{ }^{*} p i-r i{ }^{20}(\mathrm{XAV} p i / p i-r i)$

PSJ *pêj > KGG pêj

PJ *prãm' 'hungry, to want':

PCerr “prãm’ > PNJ *prãm; PNR pjãnŭ 'to want'; PCJ *mrãm // *mrã 'hungry' (XAV, XER mrãm // mrã)

PSJ “prã $\eta$ 'hungry; year' (probably via 'hunger season') > KGG prã

PJ *pry 'coal, ember':

PCerr *pra 'ember' > PNJ *pra; PCJ *pre (XER pre-hika 'glowing embers', pre-nĩzuri 'spark', pre-zapdo 'ember')

PSJ *prâñ > KGG prañ; LKL plañ

PJ *pry ( *pryn) 'road':

PCerr *pry ( * pryn) > PNJ *pry; PNR pjy

PSJ ${ }^{*} \tilde{a}-p r y /{ }^{*} \mathrm{NP} j a-p r y>$ KGG $\tilde{e}-p r y / \mathrm{NP} j a-p r y$

PJ *prũ 'wife':

PCerr *prõ > PNJ *prõ; PCJ * mrõ 'spouse, to marry' (XAV, XER mrõ)

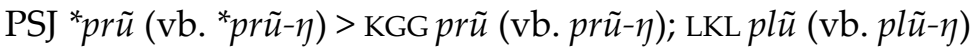

$\mathrm{PJ}{ }^{*} m b a$ 'to hear, to understand':

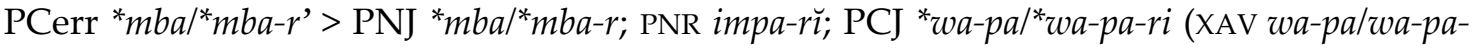
ri, XER wa-pa/wa-pa-r(i))

PSJ *mã ${ }^{*} m \tilde{a}-\eta>$ KGG $m \tilde{e} / m \tilde{e}-\eta ;$ LKL $m \tilde{a} / m \tilde{a}-\eta$

${ }^{20}$ The expected non-finite form would be ** pi-ki //**pi. Northern Jê is considered to be more conservative than Central Jê here because the Southern Jê cognate appears to correspond to the Northern Jê non-finite form. 
PJ * $m b a$ 'liver':

PCerr * $m b a>$ PNJ * $m b a$; PNR impa; PCJ * $p a(\mathrm{XAV}, \mathrm{XER} p a)$

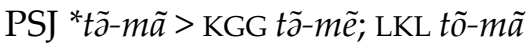

PJ *-mbay'21 'to be afraid':

PCerr * ${ }^{2}$-mba > PNJ *pymba; PNR s-umpa; PCJ *pipa (XAV, XER pipa)

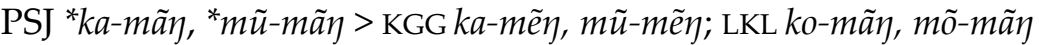

PJ *mã 'DAT':

PCerr ${ }^{*} m \tilde{a}>$ PNJ ${ }^{*} m \tilde{a} ;$ PNR $m \tilde{a} ;$ PCJ ${ }^{*} m \tilde{a}($ XAV, XER $m \tilde{a})$

PSJ * $m \tilde{a}>$ KGG $m \tilde{a} ;$ LKL $m \tilde{o}$

$\mathrm{PJ}$ * $m b \hat{a}$ 'to grab, to carry':

PCerr * $m b \hat{\partial} /{ }^{*} m b \hat{\partial}-\tilde{n}>$ PNJ *$m b \hat{\partial} /{ }^{*} m b \hat{\partial}-\tilde{n}$ 'to grab'; PNR impz-rŭ 'to carry'; PCJ *kwa-pe/*kwa-pe-j // *kwa-pe 'to carry.DU' (XAV iwa-pe/2wa-pe-j // iwa-pe, XER kwape)

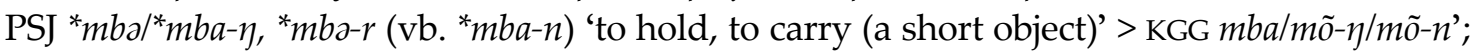
LKL $m b o / m b a-\eta, m b o l$ (vb. $m b a-n)$

PJ *mbyn 'tail':

PCerr *mbyn 'tail, penis' > PNJ *mby 'penis', *ja-mby 'tail'; PNR s-ampy 'tail'; PCJ *mãnã // *bo (XAV mãnã // ba, XER mnã // bə)

PSJ * $m b y>$ KGG, LKL $m b y$

PJ *mbyt 'celestial body':

PCerr *mbyt 'sun' > PNJ *mbyt; PNR impytĭ 'time'; PCJ *bata // *ba:da (XAV bata // ba:da, XER bta // bda)

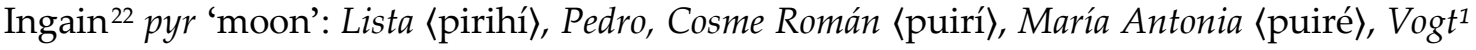
$\langle$ pyri $\rangle, \operatorname{Vog}^{3}\langle$ pyrý $\rangle$

PJ *mbec 'good':

PCerr *mbec > PNJ *mbec; PNR impe 'real'; PCJ *pêcê // *pê (XAV pêcê // pê 'well; to recover, to get better', XER pêsê // pê)

PSJ *mbe 'HABIT' > KGG, LKL mbe; PSJ * $\tilde{a}-m b e(v b . ~ * \tilde{a}-m b a-n)$ 'good weather' > KGG $\tilde{e}-m b e$ (vb. $\tilde{e}-m \tilde{o}-n)$

$\mathrm{PJ}$ * $m \tilde{e}$ 'PL; with':

PCerr ${ }^{*} m \tilde{e}>$ PNJ ${ }^{*} m \tilde{e} ;$ PNR $-m \tilde{e}-r a$ 'PL (nominal number)', $m \tilde{e}=$ 'DU (indexed on verbs)'; PCJ *mẽ 'with' (XAV mẽe, XER mẽ)

PSJ *mĩ 'PL'> LKL $m \tilde{e}$

PJ *mbên 'liquid':

PCerr *mbên > PNJ *mbế; PCJ *pĩnĩ // *pi (XAV pĩni // pĩz 'honey', XER to-pi 'eye rheum')

PSJ * $m b \hat{e}\left(\mathrm{vb} .{ }^{*} m b e-n\right)>$ KGG $m b \hat{e}(\mathrm{vb} . m b e-n)$; LKL $m b \hat{e}$

PJ *mbIn' 'husband':

PCerr *mbjan'> PNJ *mbjên; PNR impin-pja 24

PSJ *mben > KGG, LKL mben

${ }^{21} \mathrm{PSJ}{ }^{*}-\eta$ in underived stems usually goes back to PJ * $-m$ ' and corresponds to PCerr * $-m$ '. The correspondence between PSJ * $-\eta$ and PCerr zero is unparalleled; I provisionally reconstruct PJ * $-\eta$ '.

${ }^{22}$ Hereinafter, Lista refers to data from Lista 1883; Pedro, Cosme Román, and María Antonia are the names of Ambrosetti's (1896) consultants who provided the respective pieces of data; $\operatorname{Vog}^{1}{ }^{1}$, Vogt ${ }^{3}$ refer to two original wordlists contained in Vogt 1904.

${ }^{23}$ The expected utterance-final allomorph would be **pi. One possible explanation for this irregular reflex is an analogical extension of nasality from the regular utterance-medial allomorph, pĩn.

${ }^{24}$ It is unclear whether the Panará reflex of PCerr *-ja- is regular. 
PJ *mũ 'to go.PL':

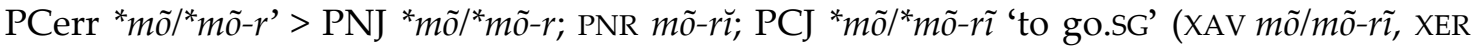
$m \tilde{o} / m \tilde{o}-r(\tilde{\imath}))$

PSJ ${ }^{*} m \tilde{u}\left(v b .{ }^{*} m \tilde{u}-n\right)>$ KGG $m \tilde{u}(v b . m \tilde{u}-n) ;$ LKL $m \tilde{u}$

PJ *mbra 'ashes':

PCerr *mbro > PNJ *mbro; PCJ *wêdê-pro 'coal' (XAV wêdê-pro, XER wdê-pro), *ka jaj-mpro 'foam' (XAV Za jaj-pro, XER ka zaĩ-pro ka zam-pro), *jadaj-mpro 'saliva' (XAV jadaj-pro, XER zdaĩ-pro), (?) *pro 'to burn' (XER pro)

PSJ *mrã > KGG $m r \tilde{e} j ;{ }^{25}$ LKL $m l \tilde{a}$

PJ *mbrom' 'ant':

PCerr *mbrum'> PNJ *mbrüm

PSJ *mbroy-jy 'Argentine ant' > KGG mbroy-jy

$\mathrm{PJ}$ * $m b r e$ 'relative by marriage' (kinship term):

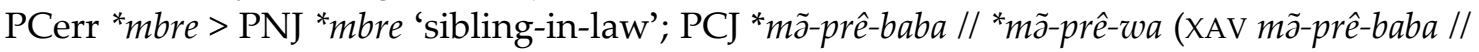
$m \tilde{\partial}-p r \hat{e}-w a$ 'mother/father-in-law', xER mã-prê-wa)

PSJ *ja-mbre > KGG ja-mbre 'cousin, son-in-law'; LKL jo-mble 'brother-in-law, father-in-law, mother-in-law'

PJ *wa 'to walk':

PCerr * wa > PNJ * $b a$ 'to walk, to live'; PNR $p a$

PSJ *wa 'STAT' (aspect marker) > KGG wẽ ; LKL $v \tilde{a}$

PJ *wy 'to take, to carry':

PCerr *wy/*wy-r'> PNJ *by/*by-r; PNR $p y-r \breve{\imath}$

PSJ ${ }^{*} w a /{ }^{*} w a-\eta /{ }^{*} w \tilde{a}-n \sim{ }^{*} w y-n{ }^{26}$ 'to hold, to carry (a long object)' > KGG $w a / w \tilde{o}-\eta / w \tilde{o}-n \sim w y-n$; LKL va/võ- $\eta / v \tilde{o}-n \sim v u-n$

PJ *wô 'to untie':

PCerr ${ }^{*} w \hat{o} /{ }^{*} w \hat{o}-\tilde{n}$ '> PNJ ${ }^{*} b \hat{o} /{ }^{*} b \hat{o}-\tilde{n}$; Southern Kayapó 〈tipó〉 (likely pô)

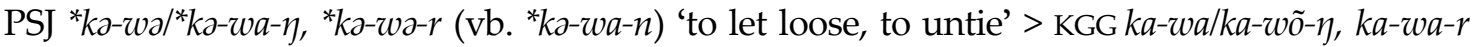
(vb. $k a-w \tilde{o}-n)$

$\mathrm{PJ}$ *ta 'to fly.SG':

PCerr ${ }^{*}$ to/*top-r > PNJ ${ }^{*} t o /{ }^{*}$ to- $r$; PNR to:/to: $-j$ 'to fly, to dance'; PCJ ${ }^{*}$ tob-ro (XAV tob-ro 'to descend', XER tb-ro 'to cross a body of water')

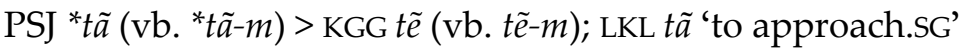

PJ *ñücta 'tongue':

PCerr * $\tilde{n} \tilde{o} j t o>$ PNJ * $\tilde{n} \tilde{o} t o ;$ PNR s-õto; PCJ * $\tilde{n} \tilde{o} j t o(X A V \tilde{n} \tilde{o} j t o \sim \tilde{n} \tilde{o t t o}$, XER nõjto)

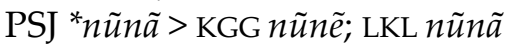

PJ *tã 'INSTR'

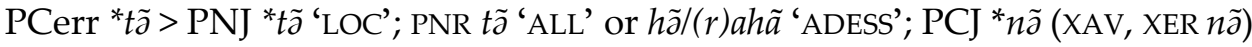

PSJ *tã 'ERG, INSTR' > KGG tã; LKL tõ

PJ *tât 'strong, hard':

PCerr ${ }^{*} t \hat{a} t>\mathrm{PNJ}{ }^{*} t a \hat{c}$; PNR tatu; PCJ ${ }^{*} t \hat{e} t \hat{e} / /{ }^{*} t \hat{e}: d \hat{e}(\mathrm{XAV}-t \hat{e} t \hat{e} / / t \hat{e}: d \hat{e}, \mathrm{XER} t \hat{e} t(\hat{e}) \sim t t \hat{e},-t \hat{e} t(\hat{e}) \sim-t t \hat{e} / /$ $-t d \hat{e})$

PSJ ${ }^{*} \operatorname{tar}\left(\mathrm{vb} .{ }^{*} t a-n\right)>$ KGG tar (vb. tõ- $\left.n\right)$; LKL tol

PJ *ty 'to die':

25 The accretion of Kaingáng * $j$ is irregular.

26 The relation of PSJ * $w y$ - $n$ to the remaining forms is not a regular one; it might be the case that two distinct etymologies are conflated here. Moreover, the Laklãnõ reflex unexpectedly shows a rounded vowel. 
PCerr ${ }^{*} t y(r) /{ }^{*} t y-k^{27}>$ PNJ ${ }^{*} t y /{ }^{*} t y-k$; PNR ty; PCJ ${ }^{*} d a r a /{ }^{*} d a-k \partial / /{ }^{*} d a(X A V$ dara/da-2a // da, XER $d a r a / d-k a / / d a)$

PSJ *ty 'to die.STAT' > KGG ty 'numb'; LKL ty

PJ *k(r)Vmtym' 'capybara':

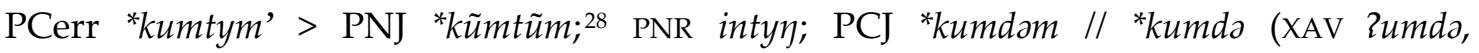
XER kumdam // kumda)

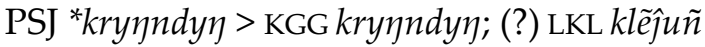

PJ *tyk 'black, dark':

PCerr *tyk 'black' > PNJ *tyk; PNR ka-ty;; PCJ *daka // *da (XAV 2rã-da, XER dka 'dark')

PSJ *ku-ty (vb. *ku-ty- $\eta$ ) 'dark, night' > KGG, LKL $k u-t y$ (vb. $k u-t y-\eta)$

PJ *tom' 'fat':

PCerr *twam'> PNJ *twâm; (?) PNR tumã; PCJ *wam // *wa (XAV wam // wa, XER rom-wa)

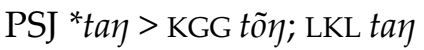

PJ *tom' 'old':

PCerr *tum' 'old' > PNJ *tũm; PNR tuך; PCJ *dum // *du 'tall' (XER dum), *dum-krata // *dum$k r a: d a$ 'elder sibling of the opposite sex' (XAV dub-2rata // dub-2ra:da, XER dum-krda)

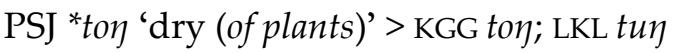

$\mathrm{PJ} * t u$ 'to carry':

PCerr ${ }^{*} t u{ }^{*} t u-r$ ' $>$ PNJ ${ }^{*} t u /{ }^{*} t u-r$; PNR $t u-r \breve{\imath}$ 'to carry in a basket.SG'; PCJ ${ }^{*} d u{ }^{*} d u-r i$ 'to carry.SG' $(\mathrm{XAV} d u / d u-r i$, XER $d u / d u-r(i))$

PSJ *tu 'to carry on one's back, to wear' > KGG, LKL tu

PJ *potu 'horsefly':

PCerr * ${ }^{2} t u>$ PNJ * $p u c u ; 29$ PNR pu:su; PCJ *pidu (XAV, XER pidu)

PSJ *patu> KGG põtu; LKL patu

$\mathrm{PJ}{ }^{*} t \tilde{u}$ 'NEG':

PCerr ${ }^{*} t \tilde{o}>$ (?) PNR rõ 'privative; negation in non-finite clauses'; PCJ *tõ ${ }^{30}$ (XAV tõ 'prohibitive; negation in purpose clauses', XER tõ 'privative')

PSJ ${ }^{*} t \tilde{u} /{ }^{*} t \tilde{u}-\eta\left(\mathrm{vb} .{ }^{*} t \tilde{u}-\eta\right)>\operatorname{KGG} t \tilde{u} / t \tilde{u}-\eta(\mathrm{vb} . t \tilde{u}-\eta) ; \operatorname{LKL} t \tilde{u} / t \tilde{u}-\eta$

$\mathrm{PJ} * t \tilde{e}$ 'to go.SG':

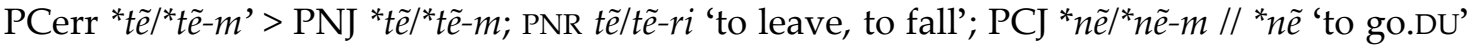

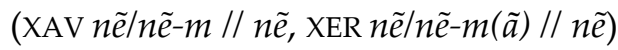

PSJ ${ }^{*} t \tilde{\imath} /{ }^{*} t \tilde{\imath}-\eta\left(\mathrm{vb} .{ }^{*} t \tilde{\imath}-n\right)>$ KGG $t \tilde{\imath} / t \tilde{\imath}-\eta(\mathrm{vb} . t \tilde{\imath}-n) ;$ LKL $t \tilde{e} / t \tilde{e}-\eta(\mathrm{vb} . t \tilde{e}-n)$

PJ *peti 'to dream':

PCerr ${ }^{*} p^{\curvearrowright} t i{ }^{*} p^{\curvearrowright} t i-r$ ' > PNJ * $p y t i /{ }^{*} p y t i-r$; Southern Kayapó (iúpintín〉 (likely $p y t i-\eta$ )

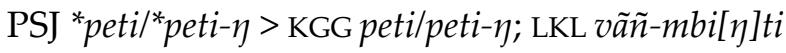

PJ *janda 'to send':

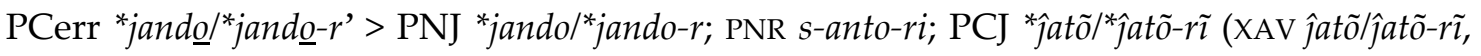
XER zatõ/zatõ-r( $(\tilde{\imath}))$

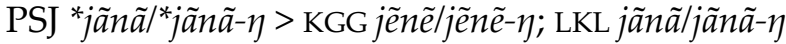

PJ *ndam 'eye':

PCerr *ndom > PNJ *ndo(p-); PNR into; PCJ *tõmõ // *to (XAV tõmõ/to, XER tmõ/to)

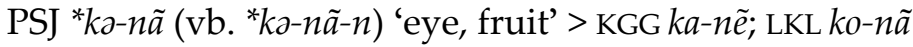

${ }^{27}$ Northern Jê languages point to PCerr finite *ty; Central Jê languages point to PCerr finite *tyr.

28 The rounding of PCerr * $y$ in PNJ is irregular.

${ }^{29}$ Medial ${ }^{*}-c$ - in place of the expected ${ }^{* *}-t$ - is irregular.

${ }^{30}$ It is unclear why PCerr *t yields PCJ *t in a nasal environment here. The expected reflex would be PCJ **no. 
PJ *nã 'mother':

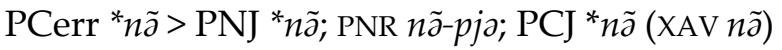

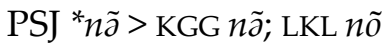

PJ *nõ 'to lie.sG':

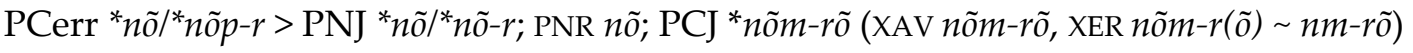

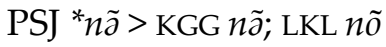

PJ *pondv ( *pondon) 'bad or crooked':

PCerr * $p^{2} n d u\left(\sim{ }^{*} p^{2} n d u n\right)$ 'bad'> PNJ *pundu

PSJ *pandô (vb. *pando-n) 'crooked'> KGG põndô (vb. põndo-n); LKL pandô

PJ * $r a$ 'to hit':

PCerr ${ }^{*}-r a /{ }^{*}-r a-\tilde{n}{ }^{\prime}>$ PNJ ${ }^{*} k u-r a l{ }^{*} k u-r a-\tilde{n}$

PSJ ${ }^{*} r \tilde{a} /{ }^{*} r \tilde{a}-\eta /{ }^{*} r \tilde{a}-n>$ KGG $r \tilde{e} / r \tilde{e}-\eta / r \tilde{e}-n ;$ LKL $l \tilde{a} / l \tilde{a}-\eta / l \tilde{a}-n$

PJ *jara 'wing; armpit':

PCerr *jara > PNJ *jara 'wing', *jara-kre 'armpit'; PNR s-aja 'wing, feather', s-a-kre 'armpit'

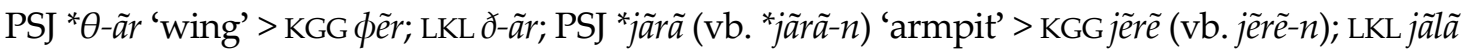
PJ *roñ 'celestial body':

PCerr *rwaj' 'moon' > PNJ *mbyt-rwâ; PCJ *waj // *wa (XER waj // wa)

PSJ *ra 'sun' > KGG rõ; LKL la 'sun, day'

PJ * $r u$ 'to transport liquid or to pour':

PCerr ${ }^{*} r u /{ }^{*} r u-\tilde{n}$ ' $>$ PNJ ${ }^{*} r u /{ }^{*} r u-\tilde{n}$ 'to spill, to pour'

PSJ ${ }^{*} r u /{ }^{*} r u-\eta /{ }^{*} r u-n$ 'to fetch water'> KGG $r u / r u-\eta / r u-n$; LKL $l u-n$ 'well'

$\mathrm{PJ}$ *re 'to leave, to abandon':

PCerr ${ }^{*} r \underline{e}^{31}>\mathrm{PNJ}{ }^{*} r e /{ }^{*} r e-(r) ; \mathrm{PCJ}{ }^{*} r \tilde{e} /{ }^{*} r \tilde{e}-m \tilde{e}(\mathrm{XAV} r \tilde{e} / r \tilde{e}-m \tilde{e}, \mathrm{XER} r \tilde{e} / r \tilde{e}-m(\tilde{e}) \sim r-m \tilde{e})$

PSJ ${ }^{*} r e /{ }^{*} r a-\eta>$ KGG $r e / r \tilde{o}-\eta$

PJ * $r \tilde{e}$ 'to throw.PL'

PCerr ${ }^{*} r \tilde{e} /{ }^{*} r \tilde{e}-\tilde{n}$ '> PNJ ${ }^{*} r \tilde{e} /{ }^{*} r \tilde{e}-\tilde{n}$

PSJ ${ }^{*} r \tilde{l} /{ }^{*} r \tilde{l}-\eta /{ }^{*} r \tilde{l}-\tilde{n}>$ KGG $r \tilde{l} / r \tilde{\imath}-\eta / r \tilde{l}-\tilde{n}$ 'to carry.PL'; LKL le $/ l \tilde{e}-\eta / l \tilde{e}-\tilde{n}$

PJ *jarê ( *jarên) 'root':

PCerr *jarê ( *jarên) > PNJ *jarê; PNR s-arê

PSJ ${ }^{*} j a \tilde{r} \hat{e}\left(\mathrm{vb} .{ }^{*} \theta-a r e-n\right)>$ KGG jõrê; LKL jãlê (vb. ð-ale-n)

$\mathrm{PJ}{ }^{*}-r \hat{e}(C)$ 'to weed':

PCerr ${ }^{*}-r \hat{e}(C) /{ }^{*}-r \hat{e}(C)-r>\mathrm{PNJ}{ }^{*} k a-r \hat{e} /{ }^{*} k a-r \hat{e}-r$

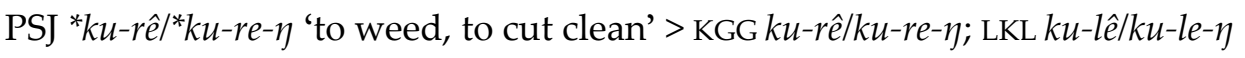
PJ *rit 'to look':

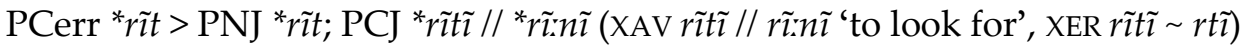

PSJ "rĩr 'to wake up, to be alive' > KGG rĩr; LKL lêl

PJ *cy 'to weave':

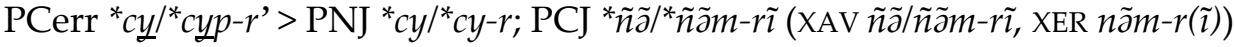

PSJ ${ }^{*} \theta y>$ KGG $\phi y ;$ LKL $\partial y$

PJ *cym 'seed':

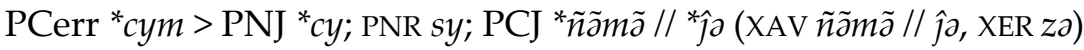

PSJ ${ }^{*} \theta y\left(\mathrm{vb} .{ }^{*} \theta y-n\right)>$ KGG $\phi y(\mathrm{vb} . \phi y-n) ;$ LKL $\partial y$

$\mathrm{PJ}{ }^{*} \mathrm{c} \hat{o}$ 'to eat soft food, to suck':

PCerr ${ }^{*} c \hat{o} /{ }^{*} c \hat{o}-r$ ' $>$ PNJ ${ }^{*} c \hat{o} /{ }^{*} c \hat{o}-r ;$ PNR sôw-rŭ

PSJ *pe- $\theta$ a (vb. * $p e-\theta a-m)$ 'to suck breast'> KGG $p a-\phi a^{32}$ (vb. $\left.p a-\phi \tilde{o}-m\right)$; LKL $p e-ð o$ (vb. $\left.p e-ð a-m\right)$

\footnotetext{
${ }^{31}$ It is unclear how the non-finite form of this verb should be reconstructed.
} 
PJ *côj 'leaf':

PCerr *côj'> PNJ *cô; PNR parĭ-sô; PCJ *cuj // *cu (XAV wê-cuj-ra, -cuj // -cu, XER su)

PSJ ${ }^{*} \theta e j\left(\right.$ vb. $\left.{ }^{*} \theta e-\tilde{n}\right)>$ KGG $\phi e j ;$ LKL ðej

PJ *jacê *jVmcê 'nest':

PCerr ${ }^{*} j a c \hat{e}>$ PNJ *jacê; PNR s-asê; PCJ *jaci (XAV jaci, XER zasi)

PSJ ${ }^{*} j \partial \eta \theta \hat{e}\left(\mathrm{vb} .{ }^{*} j \partial \eta \theta e-\tilde{n}\right)>\mathrm{KGG} j a \eta \phi \hat{e}(\mathrm{vb} . j a \eta \phi e-\tilde{n})$

$\mathrm{PJ}{ }^{*}-c \tilde{\imath}(C)$ 'bandage, sling':

PCerr ${ }^{*}-c \tilde{\imath}(C)>$ PNJ ${ }^{*} j a-c \tilde{l},{ }^{*} k a-c \tilde{\imath}$

PSJ *- $\theta \tilde{\imath}(\mathrm{vb} .-\theta \tilde{\imath}-n)$ 'bundle, bandage' > KGG $-\phi \tilde{\imath}(\mathrm{vb} .-\phi \tilde{\imath}-n)$; LKL - $\partial \tilde{e}(\mathrm{vb} .-\partial \tilde{e}-n)$

PJ *kapnja 'to chew':

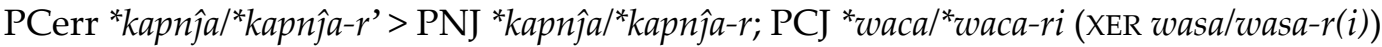

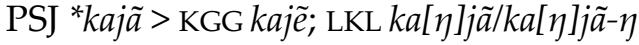

PJ *njy 'bee, bumblebee':

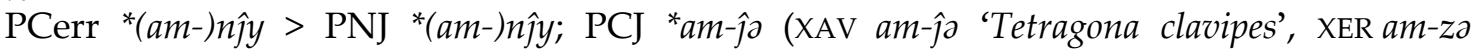

'Brazilian wasp (Protonectarina sylveirae)')

PSJ * $c y>$ KGG $x y$; LKL $c y$

PJ * $n \hat{\jmath} \hat{o}$ 'to hang.SG':

PCerr ${ }^{*} n \hat{j} \underline{\hat{o}} /{ }^{*} n \hat{\jmath} \underline{\hat{o}} p-r$ ' > PNJ ${ }^{*} n \hat{\jmath} \hat{o} /{ }^{*} n \hat{j} \hat{o}-r$; PCerr ${ }^{*} j a-n \hat{\jmath} \underline{\hat{o}} /{ }^{*} j a-n \hat{j} \underline{\hat{o}} p-r$ ' 'to hang.PL' > PNJ ${ }^{*} j a-n \hat{\jmath} \hat{o} /$

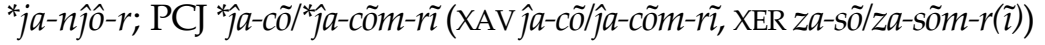

PSJ ${ }^{*} c \partial /{ }^{*} c a-\eta\left(\mathrm{vb} .{ }^{*} c \partial-m\right)>$ KGG $x a / x \tilde{a}-\eta($ vb. $x a-m) ; 33$ LKL $c o / c a-\eta$

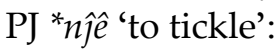

PCerr ${ }^{*} n \hat{\jmath} \hat{e} /{ }^{*} n \hat{\jmath} \hat{e}-r$ '> PNJ ${ }^{*} n \hat{\jmath} \hat{e} /{ }^{*} n \hat{\jmath} \hat{e}-r$

PSJ ${ }^{*} k u-c \hat{e} /{ }^{*} k u-c e-\eta>$ KGG $k u-x \hat{e} / k u-x e-\eta ;$ LKL $k u-c \hat{e} / k u-c e-\eta$

PJ *ja 'to stand.sG':

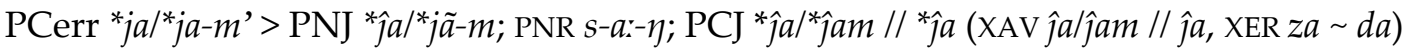

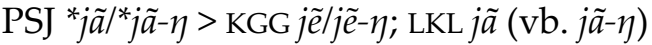

PJ * $j a$ 'to put vertically.SG'

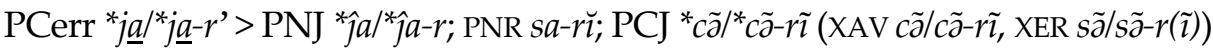

PSJ *jã (vb. $\left.{ }^{*} j \tilde{a}-\eta\right)$ 'to be put vertically’ > KGG $\phi-\tilde{e}(\mathrm{vb} . \phi-\tilde{e}-\eta)$; LKL vb. $j \tilde{a}-\eta, 3 \delta-\tilde{a}-\eta$

PJ *ñija 'nose':

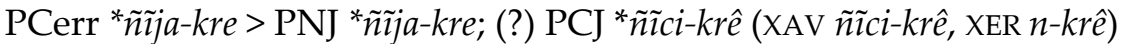

PSJ *ñ̄jã > KGG nĩjẽ; LKL nẽjã

PJ *jañ 'to eat.INTR, food'

PCerr " ${ }^{*} a j '>$ PCJ ${ }^{*} c a j / /{ }^{*} c a$ (XAV caj // ca, XER saj // sa)

PSJ ${ }^{*} j \tilde{a} /{ }^{*} j \tilde{a}-\eta /{ }^{*} j \tilde{a}-n$ (vb. $\left.{ }^{*} j \tilde{a}-n\right)>$ KGG ${ }^{*} j \tilde{e} /{ }^{*} j \tilde{e}-\eta / * j \tilde{e}-n$ (vb. jẽ-n); LKL jã $n$

PJ *jô 'bitter':

PCerr ${ }^{*} \hat{\jmath}>$ PNJ ${ }^{*} \hat{\jmath} \hat{a} ;$ PCJ ${ }^{*} \hat{\jmath} e(X A V \hat{\jmath} e$, XER $z e)$

PSJ ${ }^{*} \theta-a\left(\right.$ vb. $\left.{ }^{*} \theta-a-\eta\right)>$ KGG $\phi-a($ vb. $\phi-\tilde{o}-\eta) ;$ LKL $\partial-o($ vb. $\partial-a-\eta)$

PJ * $\tilde{n} \tilde{j} \hat{a}$ 'smoke':

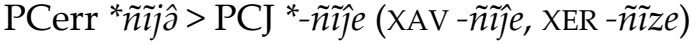

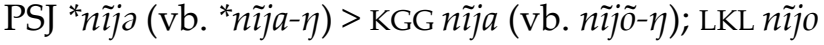

PJ *jr 'urine':

PCerr *ja > PNJ *îa; PCJ *îe (XAV je, XER ze 'bladder')

PSJ ${ }^{*} j \hat{\partial}-\tilde{n}$ 'to urinate' > KGG jo- $\tilde{n}$; LKL $j a-\tilde{n}$

32 The expected form would be KGG ${ }^{*} p e-\phi a\left(\mathrm{vb} .{ }^{*} p e-\phi \tilde{o}-m\right)$, given both the Laklãnõ cognate and the Kaingáng plural pi- $\eta-\phi a(\mathrm{vb} . p i-\eta-\phi \tilde{o}-m)$.

${ }^{33}$ Stative KGG $x a w$ 'to hang' is apparently a non-etymological formation, back-derived from $x a-m$. 
PJ * $\tilde{n} \tilde{y}$ 'to sit.SG':

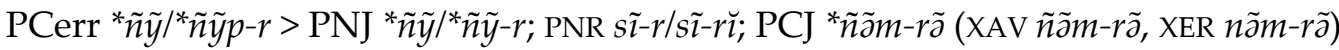

PSJ ${ }^{*} n \tilde{\imath}\left(\mathrm{vb} .{ }^{*} n \tilde{\imath}-m\right)>$ KGG $n \tilde{\imath}(\mathrm{vb} . n \tilde{\imath}-m)$; LKL $n \tilde{e}(\mathrm{vb} . n \tilde{e}-m)$

PJ *jo 'tooth':

PCerr ${ }^{*} j w a>$ PNJ ${ }^{*} \hat{w} w a$; PNR swa; PCJ ${ }^{*} k w a(X A V$, XER $k w a)$

PSJ *ja> KGG jõ ; LKL ja

$\mathrm{PJ}{ }^{*} k V j o$ 'salt':

PCerr ${ }^{*} k V j w a>$ PNJ ${ }^{*} k a \hat{\jmath} w a ;$ PCJ ${ }^{*} k V k w a$ ((?) XAV $\imath \imath \imath w a-w a: h \partial$, XER kəkwa-ra)

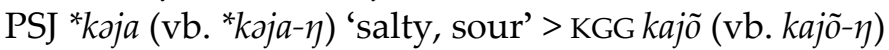

$\mathrm{PJ} * j \hat{o}$ 'to tear, to rip':

PCerr ${ }^{*}-j \hat{o} /-j \hat{o}-r$ ' $>$ PNJ ${ }^{*} k a-\hat{\jmath} \hat{o} /{ }^{*} k a-\hat{\jmath} \hat{o}-\tilde{n}$

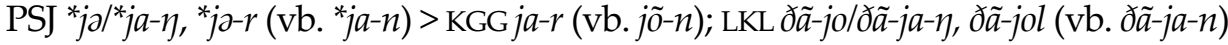

PJ *jv 'pus':

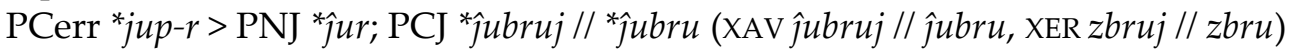

PSJ ${ }^{*} \theta-\hat{o}\left(\mathrm{vb} .{ }^{*} \theta-o-m\right)>$ KGG $\phi-\hat{o}(\mathrm{vb} . \phi-o-m) ;$ LKL $\partial-\hat{o}(\mathrm{vb} . ð-u-m)$

PJ *jv, postposition:

PCerr *ju > PNR su: 'looking for'; PCJ * $\hat{j}^{34}$ 'looking for; for' (XAV $\hat{o} \hat{o}$, XER zô)

PSJ *jô 'in front of' > KGG jô 'in front of; else'; LKL $j \hat{o}$ 'in front of'

PJ *jom' 'father':

PCerr ${ }^{*} j u m$ '> PNJ ${ }^{*} \hat{j} \tilde{u}(m)$; PNR jum-pja

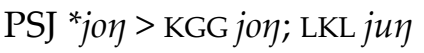

PJ * peju 'to hide':

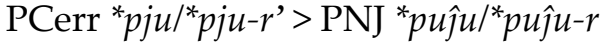

PSJ *peju > KGG peju; LKL peju

PJ * $n \hat{\jmath} u$ 'to heat, to dry':

PCerr ${ }^{*} n \hat{\jmath} u /{ }^{*} n \hat{\jmath} u-\tilde{n}$ ' > PNJ ${ }^{*} n \hat{\jmath} u /{ }^{*} n \hat{\jmath} u-\tilde{n}$ 'to dry in the sun'; Southern Kayapó 〈timuçúnkuátú, kuatáunçún $\rangle$ (likely $n s u-\eta$ )

PSJ * $c u-r$ (vb. $\left.{ }^{*} \mathrm{cu}-n\right)$ 'to heat by the fire' > KGG cur (vb. cu-n); LKL cul 'cooked half';

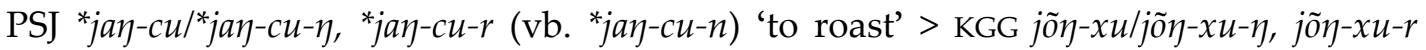

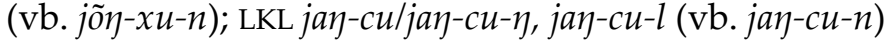

PJ * $\tilde{t} t{ }^{*} \tilde{n} \tilde{u} t$ 'to sleep':

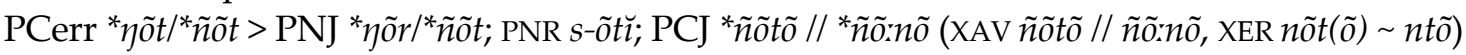

PSJ *nũr > KGG nũr; LKL nũl

PJ *jê ( *jên) 'thread, knot, cloth':

PCerr *jê $\left(\sim{ }^{*} \hat{e} n\right)>$ PNJ *jế; PNR sê

PSJ vb. ${ }^{*} \theta e-n$ 'to spin thread'> KGG $\phi e-n$; LKL $\partial e-n$

$\mathrm{PJ}{ }^{*} \tilde{n} \tilde{u} m j \hat{e}(C)$ 'female breast':

PCerr ${ }^{*} \tilde{n} \tilde{o} m \hat{\jmath} \hat{e}(C)>$ PNJ ${ }^{*} \tilde{n} \tilde{o} m \hat{\jmath} e$

PSJ *nũ $\eta j \hat{e}$ 'female breast, milk' > KGG nũ $\eta j \hat{e} ;$ LKL nũ $n \hat{\jmath} \hat{e}$

PJ *kujêk 'vein':

PCerr *kujêk > PNJ *kujêk

PSJ *kujêj > KGG, LKL kujêj

$\mathrm{PJ} * j i$ 'to lay.SG':

PCerr ${ }^{*} j i / * j i-r$ ' $>$ PNJ * ${ }^{*} i /{ }^{*} \hat{j i}-r ;$ PCJ ${ }^{*} h i / * h i-r i(X A V h i / h i-r i$, XER hi/h(i)-ri)

PSJ ${ }^{*} j i /{ }^{*} j i-\eta>$ KGG $\phi-i / \phi-i-\eta$; LKL $j i / j i-\eta$

${ }^{34}$ The expected reflex would be PCJ ${ }^{*} \hat{\jmath} u$. Note that the vowel $\hat{o}$ is extremely rare in Central Jê, its occurrence being restricted to a few function words. 
PJ *jiji * $\tilde{n} \tilde{i} i$ 'name':

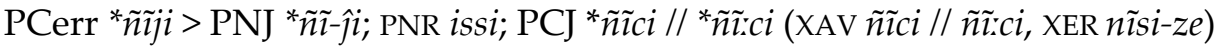

PSJ *jiji *jyjy (vb. *jiji-n *jyjy-n)> KGG jiji jyji jyjy (vb. jiji-n); LKL jyjy, 3 d-yjy (vb. jyjy-n, $3 \delta-y j y-n)$

PJ * $\tilde{n} \tilde{\imath}$ 'meat':

PCerr ${ }^{*} \tilde{n} \tilde{\imath}>$ PNJ ${ }^{*} \tilde{n} \tilde{l} ;$ PNR $\tilde{n} \tilde{l} ;$ PCJ ${ }^{*} \tilde{n} \tilde{l}(X A V \tilde{n} \tilde{l}$, XER $n \tilde{\imath})$

PSJ ${ }^{*} n \tilde{\imath}>$ KGG $n \tilde{\imath} ;$ LKL $n \tilde{e}$

PJ * $\tilde{n} \tilde{\imath}(m)-$ 'hand (in compounds)':

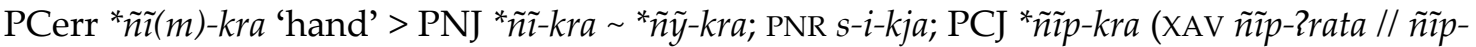
Zra:da, XER nĩp-kra)

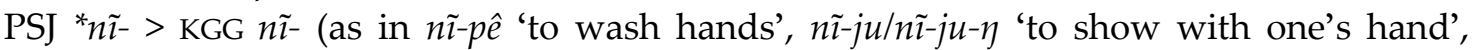

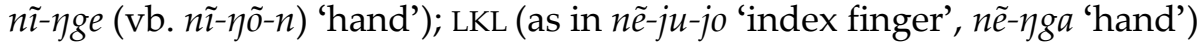

$\mathrm{PJ}{ }^{*} k a j$ ' 'basket':

PCerr *kac > PNJ *kac; (?) PNR kay

PSJ ${ }^{*} k \tilde{a} j\left(\right.$ vb. $\left.{ }^{*} k \tilde{a} \tilde{n}\right)>$ KGG $k \tilde{e} j$; LKL $k \tilde{a} j$

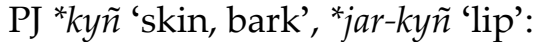

PCerr *kyj' 'skin, bark' > PNJ *ka; PNR ka; PCJ *haj // *ha (XAV ha, XER haj hê // ha); PCerr *jaj-kyj' 'lip' > PNJ *ja-ka; PNR s-a-ka; PCJ *îaj-haj // *jaj-ha (XAV jaj-haj // jaj-ha, XER zaj-ha 'buttocks')

PSJ *jãn-ky (vb. *jãn-ky-n) 'mouth' > KGG jẽn-ky (vb. jẽn-ky-n); LKL jãn-ky

$\mathrm{PJ}$ *ko 'to dig':

PCerr ${ }^{*} k w a /{ }^{*} k w a-\tilde{n}$ '> PNJ ${ }^{*} k w \hat{\partial} /{ }^{*} k w \hat{\partial}-\tilde{n} ;$ PNR $k w \partial-\eta$

PSJ ${ }^{*} k a /{ }^{*} k a-\eta\left(v b .{ }^{*} k a-n\right)>$ LKL $k a / k a-\eta(v b . k a-n)$

PJ *jar-ko 'mouth':

PCerr ${ }^{*} j a d-k w a>$ PNJ *jar-kwa; PNR s-a-kua; PCJ *jada-wa (XAV jada-wa, XER zda-wa 'mouth, door')

PSJ *jãn-ka 'door' > KGG jõn-kõ; LKL $ð-\tilde{a} n-k a$

PJ *kVñko 'sky':

PCerr *kajkwa > PNJ *kajkwa; PCJ *hajwa (XAV hojwa, XER hojwa hewa)

PSJ *kañka > KGG kañkõ; LKL koñka

PJ *kôp 'fly, mosquito':

PCerr *kôp 'fly' > PNJ *kôp; PCJ *kupu // *ku:bu (XAV $\{u p u / / ~ \imath u: b u$, XER kpu // kbu)

PSJ *ka 'mosquito' > KGG ka; LKL cẽ-to-ko 'black fly'

PJ *kôm 'tree, horn'; * $\tilde{n}$-kôm 'horn':

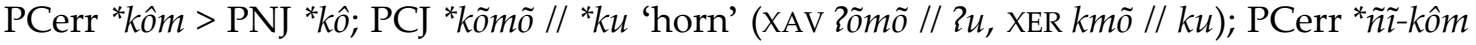
'horn' $>$ PNJ * $\tilde{n} \tilde{\imath}-k \hat{o}$

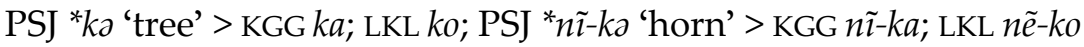

PJ *kôk *ka-kôk 'wind':

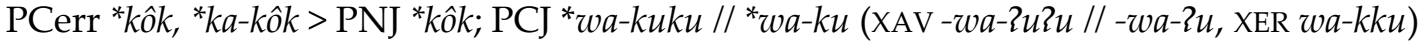

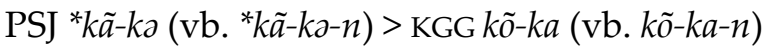

$\mathrm{PJ}$ * $k v$ 'to eat':

PCerr ${ }^{*} k u /{ }^{*} k u-r$ ' $>$ PNJ ${ }^{*} k u / k u-r$ 'to eat.PL'; PNR $k u-r \breve{r}$; PCJ ${ }^{*} h u /{ }^{*} h u-r i(X A V h u / h u-r i)$

PSJ * $k \hat{o}$ 'to eat, to use' > KGG, LKL $k \hat{o}$

$\mathrm{PJ} * k \tilde{e} t$ 'stone':

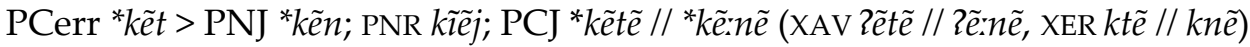

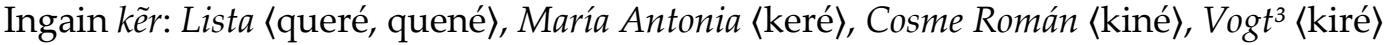

PJ *kêt 'NEG':

PCerr ${ }^{*} k \hat{e} t>$ PNJ ${ }^{*} k \hat{e} t$

PSJ *kêr 'ADVT'> KGG kêr; LKL kêl-o 
$\mathrm{PJ}{ }^{*} k_{I j}$ 'pit'

PCerr *kjaj' > PNJ *kî 'earth oven'; PNR kjê 'earth oven'

PSJ *kêj 'tomb' > KGG kêj

PJ ${ }^{*} k_{I} \sim{ }^{*} k_{I j} \sim{ }^{*} k_{I j}$ ' 'to split'

PCerr ${ }^{*}-k j a{ }^{*}-k j a-r$ '> PNJ ${ }^{*} k o-k \hat{\imath} /{ }^{*} k o-k j \hat{e}-r$

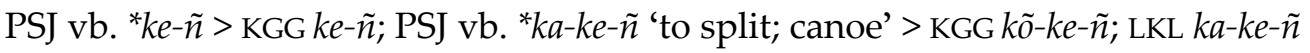

PJ *kra 'offspring':

PCerr ${ }^{*} k r a>$ PNJ ${ }^{*} k r a$; PCJ ${ }^{*} k r a: / /{ }^{*} k r a(X A V$ ใra: // $2 r a$, XER $k r a)$

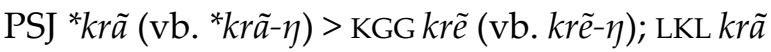

PJ *krat 'macaw':

PCJ *krata // *kra:da (XAV 2rata // 2ra:da, XER krda)

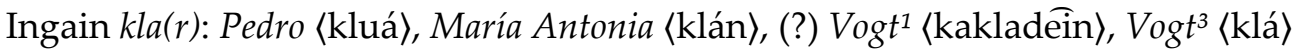

PJ *krỹ $\tilde{n}$ 'head':

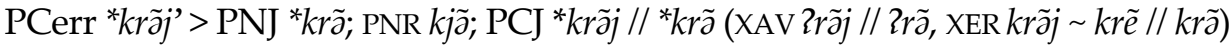

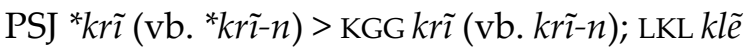

PJ *jVkrỹñ 'knee':

PCerr *jikrãj'> PCJ *hikrãj // *hikrã (XAV hißrã-ti, XER hi-krãj-ti hi-krẽ-ti, hi-krãj-)

PSJ ${ }^{*} j$ jkrĩ > KGG jakri; LKL joklẽ

PJ *krot 'chin, beak':

PCerr *krwat 'beak' > PNJ *krwât; PCJ *wata // *wa:da (XAV wata // wa:da 'chin, beak', XER wda)

Ingain jat-krar 'chin': Lista 〈amincrará〉, María Antonia 〈miet krará〉, Vogt³ 〈jitkyrará〉

PJ *kre 'hole':

PCerr ${ }^{*} k r e>$ PNJ *kre; PNR kre; PCJ *krê 'vagina, anus' (XAV $2 r \hat{e}$, XER krê), *am-krê 'hole' (XAV am-2rê, XER am-krê)

PSJ ${ }^{*} k r e>$ KGG kre; LKL kle

$\mathrm{PJ}{ }^{*} k r e$ 'to plant':

PCerr *kre > PNJ *kre; PNR kre; PCJ *krê (XAV 2rê, XER krê)

PSJ *kre (vb. *kra-n)> KGG kre (vb. krõ-n); LKL klel ${ }^{35}$ (vb. kla-n); PSJ ${ }^{*} \tilde{a}-k r e /{ }^{*} j a-k r e ~ ' p l a n t a t i o n '$ (vb. $\left.{ }^{*} \tilde{a}-k r a-n /{ }^{*} j a-k r a-n\right)>$ KGG $\tilde{e}-k r e / j a-k r o \tilde{-} n$

PJ ${ }^{*} k r i \tilde{n}$ 'thigh':

PCerr *krjaj'> PNJ *kjê; PCJ *îaj // *ja (XAV jaj // ja $a$, XER zda)

PSJ ${ }^{*} k r \hat{e}>$ KGG krê; LKL klê

$\mathrm{PJ}{ }^{*} \eta \tilde{\partial}(C)$ 'to push against, to grind':

PCerr * $\eta \tilde{a} /{ }^{*} \eta \tilde{a}-\tilde{n}$ '> PNJ * $\eta \tilde{a} /{ }^{*} \eta \tilde{a}-\tilde{n}$

PSJ * $\eta \tilde{\partial} /{ }^{*} \eta \tilde{\partial}-\eta>$ KGG $\eta \tilde{a}$

PJ *ygoñ' 'feather, hair':

PCerr * $\eta g w a \tilde{n}$ '> PNJ * $\eta g w a ̂ n \tilde{n}$ 'feather'; PNR i $\eta k w a \eta$

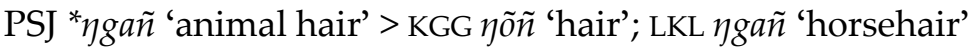

PJ * $\eta g \hat{o}$ 'louse':

PCerr * $\eta g \hat{o}>$ PNJ * $\eta g \hat{o} ;$ PNR kjã- $\eta k \hat{o} ;$ PCJ * $k u(X A V \imath u)$

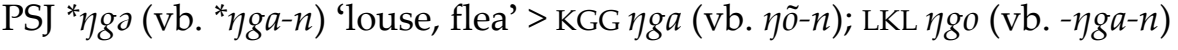

PJ * $\eta \tilde{u}$ 'to push against, to crumble':

PCerr ${ }^{*}-\eta \tilde{o} /{ }^{*}-\eta \tilde{o}-\tilde{n}^{\prime}>$ PNJ ${ }^{*} k a-\eta \tilde{o} / k a-\eta \tilde{o}-\tilde{n}$

PSJ * $\eta \tilde{u} /{ }^{*} \eta \tilde{u}-\eta>$ KGG $\eta \tilde{u} / \eta \tilde{u}-\eta$

${ }^{35}$ Final $-l$ in the Laklãnõ form is apparently non-etymological. It might have arisen as a back-derivation from the regular verbalized form kla- $n$. 
PJ * $\eta g_{I}$ 'to enter.PL':

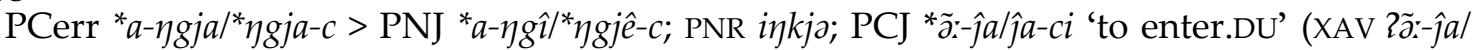
$\hat{\jmath} a-c i$, XER $z a-s(i))$

PSJ * $\eta g \hat{e}\left(\mathrm{vb} .{ }^{*} \eta g \hat{e}-m\right)>$ KGG $\eta g \hat{e}(\mathrm{vb} . \eta g \hat{e}-m)$; LKL $\eta g \hat{e}(\mathrm{vb} . \eta g \hat{e})$

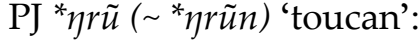

PCerr * $\eta r \tilde{o}>$ PNJ * $\eta r \tilde{o} ;$ PNR i $\eta k j \tilde{o}-p e p e t \check{\imath}$

PSJ * $\eta r \tilde{u}>$ KGG $\eta r \tilde{u} ;$ LKL $\eta l \tilde{u}$

PJ * $\eta g r e$ 'egg':

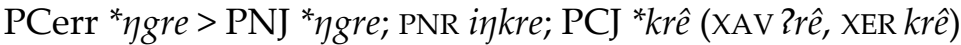

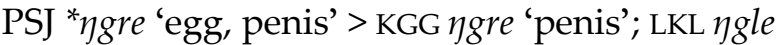

PJ * $\eta g r e$ 'to dance':

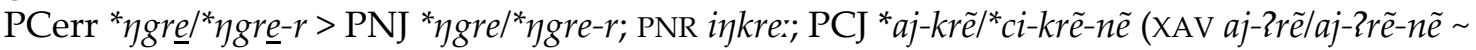
$c i-2 r \tilde{e}-n \tilde{e})$

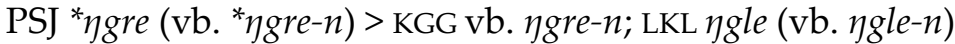

PJ * $\eta r i \tilde{\imath}(C)$ 'wrap':

PCerr * ${ }^{*} \eta \tilde{\imath}(C)>$ PNJ *ku- $\eta r \tilde{\imath}$ 'to gather in a bundle', * $-\eta r \tilde{\imath}$ 'to make packages'

PSJ * $r r \tilde{\imath} /{ }^{*} \eta r \tilde{\imath}-\eta,{ }^{*} \eta r \tilde{l}-r\left(v b .{ }^{*} \eta r \tilde{\imath}-n\right)>$ KGG $\eta r \tilde{l} / \eta r \tilde{\imath}-\eta / \eta r \tilde{\imath}-n ;$ LKL $\eta l \tilde{e} / \eta l \tilde{e}-\eta, \eta l \tilde{e}-r(v b . \eta l \tilde{e}-n)$

\section{Semantic issues}

PJ *pu:

PCerr * $p u>$ PNJ *pu 'tube (in compounds)'; PCerr “ $\eta g r w a-p u$ 'moriche stem' > PNJ * $\eta g r w a-p u$, PCJ *wa:-bu (XAV wa:-bu, XER wa-bu)

PSJ *pu (vb. * $p u-\eta)$ 'handle’ > KGG $p u$ (vb. $p u-\eta)$; LKL vb. $p u-\eta$

PJ *prit:

PCerr *prît $>$ PNJ *prĩt 'pequi'

PSJ *prĩr > KGG prĩr 'araucaria resin'; (?) LKL plẽl 'reddish' $\mathrm{PJ}{ }^{*} w \tilde{e}(C)$ :

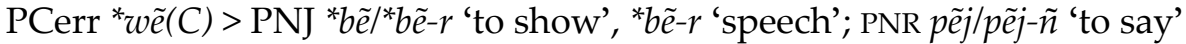

PSJ * $w \tilde{\imath}\left(\mathrm{vb} .{ }^{*} w \tilde{\imath}-n\right)$ 'to speak'> KGG $w \tilde{\imath}(\mathrm{vb} . w \tilde{\imath}-n)$; LKL $v \tilde{e}$

$\mathrm{PJ}{ }^{*} m \tilde{e}:$

PCerr ${ }^{*} m \tilde{e} /{ }^{*} m \tilde{e}-\tilde{n}$ ' 'to throw.SG' > PNJ ${ }^{*} m \tilde{e} /{ }^{*} m \tilde{e}-\tilde{n} ;$ PNR $m \tilde{e}-\tilde{n} / m \tilde{e}-r \breve{l} ;$ PCJ ${ }^{*} m \tilde{e} /{ }^{*} m \tilde{e}-j / /{ }^{*} m \tilde{e}(\mathrm{XAV}$ $m \tilde{e} / m \tilde{e}-j / / m \tilde{e}, \mathrm{XER} m \tilde{e})$

PJ *tit:

PSJ ${ }^{*} m \tilde{\imath} /{ }^{*} m \tilde{\imath}-\eta /{ }^{*} m \tilde{\imath}-n$ 'spill' > KGG $m \tilde{\imath} / m \tilde{\imath}-\eta / m \tilde{\imath}-n$

PCerr *tjat 'to burn' > PNJ *tjêr/*tjêt; PNR titŭ; PCJ * jata // *ja:da (XAV jata // ja:da, XER zata)

PSJ *têr (vb. *te-n) 'to die.ACT' > KGG têr (vb. te-n); LKL têl (vb. te-n)

PJ *rat:

PCerr *-rot $>$ PNJ * $\tilde{n} \tilde{\text {-rot }}$ 'crooked'

PSJ *rãr 'sharp-ended; thorn' > KGG rẽr; LKL lãl

$\mathrm{PJ}{ }^{*}$ ro:

PCerr ${ }^{*} r w a /{ }^{*} r w a-k>$ PNJ ${ }^{*} r \hat{u} /{ }^{*} r w \hat{\partial}-k$ 'to descend'

PSJ ${ }^{*} r a$ 'to enter.SG, to begin.SG' > KGG rõ; LKL la

PJ *kurôt:

PCerr *kurôt $>$ PNJ *kurôt 'skin irritation' (only Apinajé)

PJ *rê:

PSJ *kôrar > KGG kôrar 'frog'

PCerr $\left.{ }^{*} r \hat{e}\right|^{*} r \hat{e}(p)-r>$ PNJ ${ }^{*} r \hat{e} /{ }^{*} r \hat{e}-r$ 'to cross'

PSJ ${ }^{*} r \hat{e}\left(\mathrm{vb} .{ }^{*} r \hat{e}-m\right)$ 'to descend.PL'> KGG ${ }^{*} r \hat{e}(\mathrm{vb} . r \hat{e}-m)$; LKL lê (vb. lê-m) 
PJ *nja 'to bite': 36

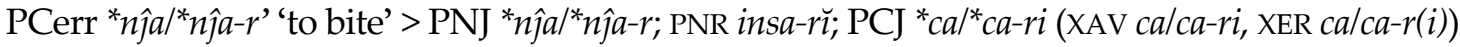

PSJ *cã 'game (animals); war' > KGG $x \tilde{e}$

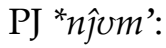

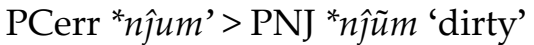

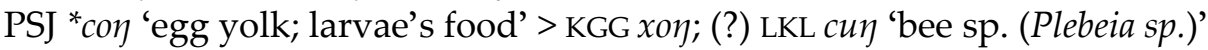

$\mathrm{PJ}{ }^{*} n \hat{\jmath} \mathrm{i}(\mathrm{C})$ :

PCerr * $n \hat{\jmath} i(C)>$ PNJ * $n \hat{\imath} i$ 'mother'; (?) PNR si-pja 'wife'

PSJ ${ }^{*} c i$ 'old'> KGG $x i$; LKL $c i$

PJ * $\eta g r \hat{:}: 37$

PCerr * $\eta g r \hat{e}>\mathrm{PNJ}$ * $\eta g r \hat{e}$ 'few'

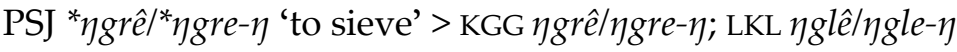

Phonetic issues

PJ *pu *pv (if PSJ is exceptional) *po (if PCerr is exceptional) 'to wrap':

PCerr ${ }^{*} k u-p u>$ PNJ ${ }^{*} k u-p u$; PCJ $\left.{ }^{*} k u-b u(X A V\} u-b u\right)$

PSJ *pa (vb. * $p a-\eta)>$ KGG $p \tilde{o}(\mathrm{vb} . p \tilde{o}-\eta)$; LKL $p a(\mathrm{vb} . p a-\eta)$

PJ *wrr' (if PSJ is exceptional) *wâr' (if PNJ/PCerr is exceptional) 'tree sp.':

PCerr *wad *wâd 'tree' > PNJ *bar; PNR par̆̈; PCJ *wêdê // *wê:dê (XAV wêdê // wề:dê, XER wdê)

PSJ *wan 'bamboo' > KGG wõn; LKL van

PJ *trm' (if PSJ is exceptional) *tâm' (if PNJ/PCerr is exceptional) 'new':

PCerr *tam' *tâm'> PNJ *tam 'raw'; PCJ *tem // *te (XAV, XER tem // te)

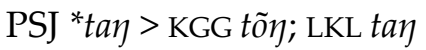

PJ *tut (if PSJ is exceptional) *ton' (if PNJ/PCerr is exceptional) 'pigeon':

PCerr ${ }^{*}$ tut $>$ PNJ ${ }^{*}$ tut

PSJ *tôn > KGG tôn

PJ *jar-râ (if PSJ is exceptional) *jar-rr (if PNJ/PCerr is exceptional) 'saliva':

PCerr *jad-râ *jad-ra > PNJ *jar-ra; PCJ *jare (XER zdare mẽ 'to spit' = 'to throw saliva')

PSJ ${ }^{*}$ jara (vb. *jara-n)> KGG jõra (vb. jõrõ-n); LKL jãlo

PJ *jVmVं 'cheek':

PCerr *jam $\tilde{V}>$ PNJ *jamã

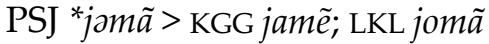

PJ *kô (if PSJ is exceptional) *ky (if PNJ/PCerr is exceptional) 'bad smell':

PCerr ${ }^{*} k \hat{a}>$ PNJ ${ }^{*} k \hat{a}$

PSJ *ky > KGG, LKL $k y$

PJ *ndon (if PSJ is exceptional) *ndun (if PNJ/PCerr is exceptional) 'snail':

PCerr *ndwan'> PNJ *ndwân; PNR intôw

PSJ *ndun > KGG ndôn nũne, ndun; LKL ndun 'caterpillar'

PJ *kvm' (if PSJ is exceptional) * $\eta g v m$ ' (if PNJ/PCerr is exceptional) 'mist, cloud':

PCerr *kum' ‘ngum'> PNJ *kũm 'smoke, mist'; PCJ *hum // *hu; PCerr *ka-kum' 'cloud, dry season' > PNJ *ka-kũm 'cloud'; (?) PNR a-kuך 'dry season'; (?) PCJ *wa-hum // *wa-hu 'dry season, year'

36 Despite the semantic divergence between the Cerrado languages and Kaingáng, which makes the comparison dubious, the Proto-Cerrado root may safely be identified as a retention from Proto-Jê, given that a secure external cognate exists in Maxakalí: /ca/ 'to bite, to sting'.

37 If this comparison is correct, the Proto-Jê word could have meant 'small' and correspond externally to Maxakalí /ktĩk-nãk/ 'small'. 


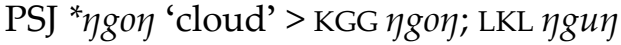

PJ * $\eta g \hat{o} j$ (if PSJ is exceptional) * $\eta g v j$ (if PNJ/PCerr is exceptional) 'water'

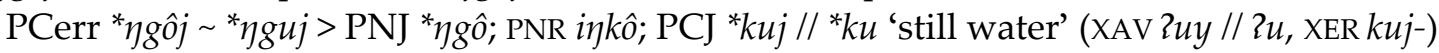

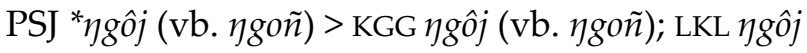

\section{Reflexes only in PCerr}

PCerr *pôr/*pôk' to ignite' > PNJ *pôr/*pôk; PNR pô 'to burn'

Possible PJ reconstruction: *pôk. External cognate: Maxakalí /pyk/ 'to burn (vi)', Rikbáktsa pok, Proto-Tupian * puk.

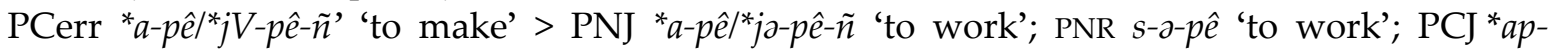

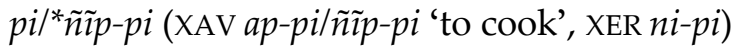

Possible PJ reconstruction: * ${ }^{*} p \hat{e}$. External cognate: Krenák pi.

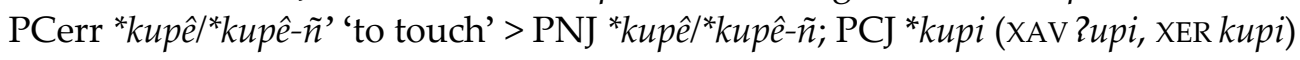

Possible PJ reconstruction: * ${ }^{*} \hat{e}$. External cognate: Maxakalí /REL-pit/.

PCerr *prồ( $j$ ') 'feather, corn husk' > PNJ *prô

Possible PJ reconstructions: *prâ, *prâj, or *prâñ. External cognate: Maxakalí /ptyc-nãk/ 'bird'.

PCerr ${ }^{*} m b r a{ }^{38}$ 'to walk.PL' > PNJ * $m b r a /{ }^{*} m b r a-r$; PCJ ${ }^{*} k m \tilde{a}-p r a /{ }^{*} k m \tilde{\partial}-p r a-b a$ 'to take away' (XAV

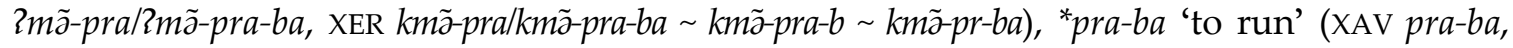
XER pra-ba $\sim p r a-b \sim p r b-a$ 'to dance')

Possible PJ reconstruction: * $m b r a(C)$. External cognate: Rikbáktsa parak 'to walk, to run.SG'.

PCerr ${ }^{*} m b w a{ }^{*} m b w a-r$ ' 'to cry' > PNJ ${ }^{*} m b \hat{u} /{ }^{*} m b \hat{\partial}-r$

Possible PJ reconstruction: *mbo. External cognates: Maxakalí /pu-t-a, IRR pu/ (Ritual

Maxakalí /pu-t, IRR pu/), Krenák pu-k, Karajá bu, Proto-Jabutí *mbo.

PCerr *-mrã (m) 'to bathe (vt.)' > PNJ *kumrã

Possible PJ reconstruction: ${ }_{-}^{*} m r \tilde{y}$ or ${ }^{*}$-mrỹm. External cognates: Maxakalí /mnỹp/

'to submerge', (?) Rikbáktsa para (unless related to PSJ *mbrô 'to swim, to bathe' > KGG $m b r \hat{o}$, LKL $m b l \hat{)}$.

PCerr * $m r \tilde{o}(C)$ 'to dive, to soak; to cook' > PNJ * $m r \tilde{o}$

Possible PJ reconstruction: ${ }^{*} m r \tilde{u}(C)$ or ${ }^{*} m r \tilde{o}(C)$. External cognates: Rikbáktsa mũrũ 'to bathe (vi)', Proto-Jabutí *mrã 'to bathe (vi)'.

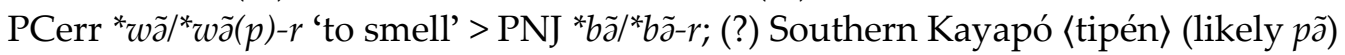

Possible PJ reconstruction: *wã. External cognates: Maxakalí /cy-pyp/ 'nose', /ca-pyp/ 'pig',

Ritual Maxakalí /byp/ 'to smell', /ca-byp/ 'pig', Krenák wap 'to smell, to kiss'.

PCerr *wôc 'to arrive' > PNJ *bôj/*bôc; PNR pôw/pôw-rŭ; (?) PCJ *wi/*wi-ci 'to arrive.SG' (XAV wi/wi-ci, XER $w i / w(i)-s i)$

Possible PJ reconstruction: *wôc. External cognate: Proto-Tupian *wuc 'to arrive (Jurúna), to go out (Káro, Puruborá)'.

PCerr *wê 'ABL' > PNJ ‘bê 'ABL, MALEF, COP'; PNR pế; PCJ *wi (XAV, XER wi)

Possible PJ reconstruction: *wê. External cognate: Proto-Tupian ${ }^{*} w i$.

PCerr *jawê 'to love, to respect' > PNJ *jabê 'to love, to be melancholy'; PCJ *jawi (XAV jawi, XER zawi)

Possible PJ reconstruction: *jawê. External cognate: Maxakalí /capit/ 'to call, to invite'.

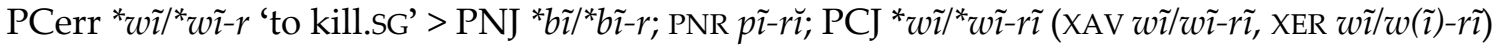

Possible PJ reconstruction: *wĩ. External cognates: Maxakalí /mĩ-k, IRR mĩ// 'to make'

(Ritual Maxakalí 'to kill'), Proto-Tupian *wĩ 'to kill'.

${ }^{38}$ It is unclear how the non-finite form of this verb should be reconstructed. 
PCerr ${ }^{*} t \tilde{j}$ ' 'brother' > PNJ *tõ; PNR tõ; PCJ *nõj // *nõ 'younger sibling of the same sex' (XAV nõj // nõ)

Possible PJ reconstruction: ${ }^{*} t \tilde{u} j,{ }^{*} t \tilde{o} j,{ }^{*} t \tilde{u} \tilde{n}$, or ${ }^{*} t \tilde{n} \tilde{n}$. External cognate: Maxakalí /nũc/.

PCerr *te 'GEN, ERG' > PNJ *te 'ERG, GEN (material, stimulus)'; PCJ *tê (XAV tê '1sG.ERG', wa-tê '1PL.ERG', (têe-)tê '3.ERG', XER tê $)$

Possible PJ reconstruction: *te. External cognates: Maxakalí /te/, (?) Krenák ti 'I', ho-ti 'you'.

PCerr *tik 'belly' > PNJ *tik; PCJ *diki // *di (XAV dili // di, XER dki // di)

Possible PJ reconstruction: *tik. External cognates: Maxakalí/tek/.

PCerr *ndap 'sour' > PNJ *ndap 'sour, ripe'; PCJ *wam-tapa 'sour, bitter' (XER wam-tap(a) wam-tpa)

Possible PJ reconstruction: ${ }^{*} n d a p$. External cognate: Proto-Tupian ${ }^{*} n d \partial p$.

PCerr *ndep 'ripe' > PNJ *ndep

Possible PJ reconstruction: *ndep. External cognate: Tuparí tep (if from Proto-Tupian *ndep).

PCerr * $p$ rak'to look like' > PNJ * pyrak

Possible PJ reconstruction: * $p$ Vryk. External cognates: Maxakalí /pytyk/.

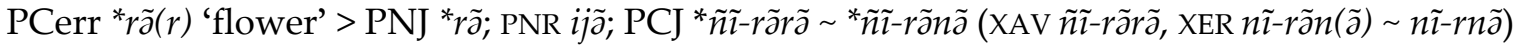

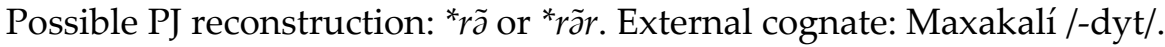

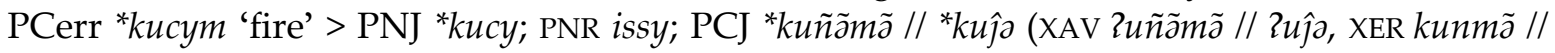
kuza)

Possible PJ reconstruction: *kucym. External cognates: Maxakalí /kycap/, Karajá he-kody.

PCerr *ci 'bone' > PNJ *ci; PCJ *hi (XAV, XER hi)

Possible PJ reconstruction: *ji or *ci. External cognates: Krenák jek, Karajá di, Ofayé hi hih,

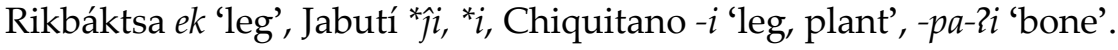

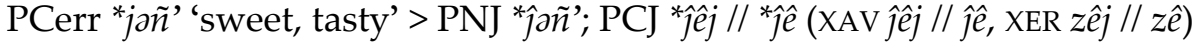

Possible PJ reconstruction: ${ }^{*} j \gamma \tilde{n}$ '. External cognates: Maxakalí /cyc-pek/, Tuparí hoc (if from

Proto-Tupian *joc *jôc).

PCerr *jwañ 'NMLZ.AG' > PNJ *înâñn, PCJ *-kwaj // *-kwa

Possible PJ reconstruction: *joñ'. External cognate: Karajá /-du/.

PCerr *jajwa/*jajwa-r' 'to lay.PL, to spill' > PNJ ${ }^{*} j a \hat{\jmath} \hat{u} /{ }^{*} j a \hat{j} w \hat{\partial}-r$; PNR s-aswa-rŭ 'to spill'; PCJ ${ }^{*} C^{-}$ $a(j) k w a /{ }^{*} c-a(j) k w a-r i(X A V c-a q w a / c-a q w a-r i$, XER s-ajkwa/s-ajkwa-r(i))

Possible PJ reconstruction: *-jo. External cognate: Maxakalí /nỹ =cu-k, IRR nỹ $=\mathrm{cu} /$.

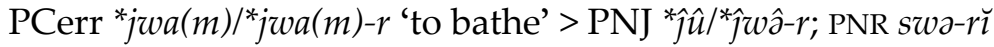

Possible PJ reconstruction: ${ }^{*}$ jom. External cognate: Krenák jum, Proto-Jabutí ${ }^{*} d z o$.

PCerr * $p^{a} j i$ 'one' > PNJ *pyji $i$ PCJ *pici (XER pici 'only')

Possible PJ reconstruction: * $p$ Vji. External cognates: Maxakalí /pycet/, (?) Krenák pucik.

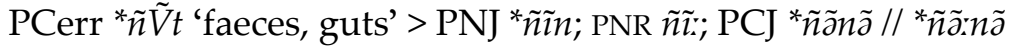

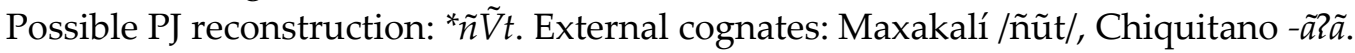

PCerr *kwar 'manioc' > PNJ *kwâr; PNR kwy

Possible PJ reconstruction: *kor. External cognate: Maxakalí /kut/.

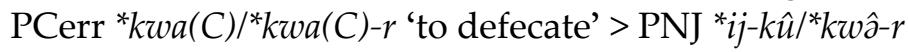

Possible PJ reconstruction: *ko(C). External cognates: Karajá $k u$.

PCerr *kryt 'metal, flint' > PNJ *kryt; PCJ *hata // *ha'da (XAV hada, hata-ra 'ax', XER hda)

Possible PJ reconstruction: *kryt. External cognate: Krenák krak 'metal, knife'.

PCerr *kroj' 'rotten' > PNJ *kro; PCJ *kroj // *kro (XAV 2roj // 2ro, XER kroj // kro)

Possible PJ reconstruction: *kraj or *krañ. External cognates: Maxakalí /ktuc/, Rikbáktsa horo.

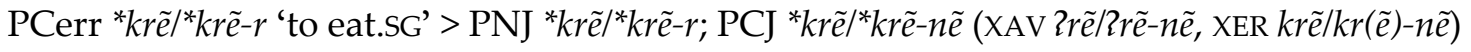

Possible PJ reconstructions: *krẽ or ${ }^{*} k r e \tilde{y}$ '. External cognate: Maxakalí /knỹk/ 'to have sex with'. 
PCerr *ngryk 'angry' > PNJ *ngryk; PCJ *həkə // *hə (XAV hə3ə // hə)

Possible PJ reconstruction: * $\eta g r y k$. External cognate: Krenák ygraך 'angry, snake'.

PCerr ${ }^{*} y a{ }^{*} j \partial p-r$ 'to roast' > PNJ * $g a{ }^{*}{ }^{*} \hat{\jmath} \partial-r ; \mathrm{PCJ}{ }^{*} \hat{\jmath} \hat{e} b-r \hat{e}(\mathrm{XAV} \hat{\jmath} \hat{e} b-r \hat{e}, \mathrm{XER} d \hat{e} b-r(\hat{e}))$

Possible PJ reconstruction: ${ }^{*} a\left({ }^{*} / a p ' /\right)$. External cognates: Maxakalí /mũ $=$ hap/ 'to roast, to burn', Krenák op.

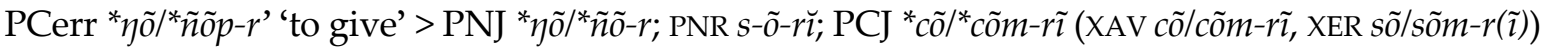

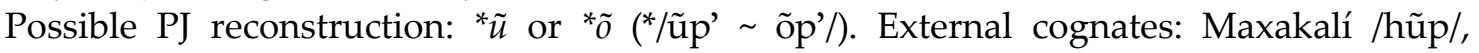
Krenák um, Karajá õ, Proto-Jabutí *u.

\section{Reflexes only in PSJ}

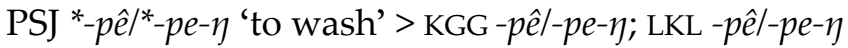

Possible PJ reconstructions: ${ }^{*}-p \hat{e}$ or ${ }^{*}-p_{I}$. External cognates: Maxakalí /pi-k, IRR pi/, Rikbáktsa $p i k$, Proto-Jabutí * $p i$.

PSJ *pra/*pra- $\eta$ 'to bite' > KGG pra/prõ- $\eta$; LKL plo/pla- $\eta$

Possible PJ reconstructions: *prô, *prôp, *prôm, *prâ, *prâp, or *prâm. External cognates: Maxakalí /ptup/, Karajá ro, Rikbáktsa boro.

PSJ *mã 'bee, honey' > KGG mã $;$; LKL mõ $\eta$

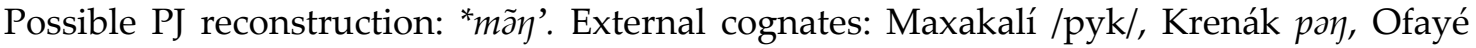
$\phi o k \sim \phi a k \sim \phi \partial k \sim \phi \partial g^{n} \sim \phi o k-t a j \sim \phi \partial g-t a j$.

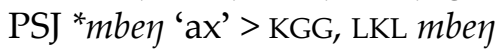

Possible PJ reconstructions: *mbêm’ or “mbım'. External cognate: Maxakalí /pip-/.

PSJ *wâ 'rotten' > KGG wa

Possible PJ reconstruction: *wrm. External cognates: Krenák wam, (?) Ofayé фa:?.

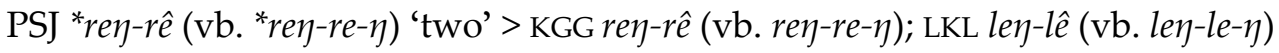

Possible PJ reconstructions: ${ }^{*}-r \hat{e}$ or ${ }^{*}-r$. External cognate: Maxakalí /tik/.

PSJ *rir 'soft (of corn)' > KGG rir

Possible PJ reconstruction: *rit. External cognate: Maxakalí /te, IRR te-t/ 'to prepare'.

PSJ ${ }^{*} \theta \partial /{ }^{*} \theta a-\eta$ 'to wash (clothes)' > KGG $\phi a / \phi \tilde{o}-\eta ;$ LKL $о / \partial a-\eta$

Possible PJ reconstructions: ${ }^{*} j \hat{o}(C),{ }^{*} j \hat{\partial}(C),{ }^{*} C \hat{o}(C)$, or ${ }^{*} c \hat{\partial}(C)$. External cognate: Ofayé $x o h$.

PSJ ${ }^{*} \theta u$ 'vagina' > KGG $\phi u$; LKL $\partial u$

Possible PJ reconstructions: ${ }^{*} j u$ or ${ }^{*} c u$. External cognate: Krenák jo, Ofayé $x a$.

PSJ *jur 'to arrive' > KGG jur; LKL jul

Possible PJ reconstruction: *jut. External cognate: Maxakalí /mũ-ca, IRR mũ-cã-t/ 'to arrive/leave.PL'.

PSJ * $k i$ 'LOC' > KGG, LKL $k i$

Possible PJ reconstruction: ${ }^{*} k i(C)$. External cognate: Karajá $k i$.

PSJ * $\eta g a$ 'earth' > KGG $\eta g a$, LKL $\eta g o$

Possible PJ reconstruction: ${ }^{*} \eta g \hat{o}(C)$ or ${ }^{*} \eta g \hat{\partial}(C)$. External cognates: Chiquitano $k y-$, ProtoTupian ${ }^{*} k u c$.

\section{Conclusions}

Above I have exposed what I believe to be the first modern proposal regarding the reconstruction of Proto-Jê phonology and basic vocabulary.

Some of the ideas advanced in this paper may appear to be somewhat radical, such as the reconstruction of five contrastive vowel heights and of two coda types; these were, however, 
the most parsimonious explanations I could find. It is nevertheless possible that an entirely different reconstruction will emerge in the future that will also account for the data.

Future research will have to tackle the issue of the unexpected nasalization in Central Jê, which remains completely unexplained in my current framework.

Another issue that remained beyond the scope of this paper is the reconstruction of ProtoJê morphosyntax. It appears possible to project many morphosyntactic features shared by Northern Jê and non-Jê Macro-Jê languages, such as Maxakalí, onto the Proto-Jê level, even though a detailed study that would take into account Central Jê and Southern Jê data has not been carried out so far. These features include:

- AOV/SV, dependent-head constituent order;

- split-S alignment in clauses headed by finite verbs; ergative-absolutive alignment in clauses headed by non-finite verbs (cf. Castro Alves 2010, Nikulin \& Silva to appear);

- obligatory expression of preposed internal arguments, leading to the occurrence of "expletive" third person markers in case of arguments dislocated from their canonical position;

- a clear-cut distinction between inflectable and non-inflectable nouns, whereby noninflectable nouns must be preceded by a possessive classifier or a genitive postposition in order to be possessed, among other features.

Inflectional and derivational morphology of Proto-Jê also deserves to be explored in greater detail. Inflectional markers must have included person prefixes (encoding the possessor in nouns, the complement in postpositions, the $\mathrm{O} / \mathrm{S}_{\mathrm{O}}$ argument in finite verbs, and the $\mathrm{O} / \mathrm{S}$ argument in non-finite verbs) and non-finiteness suffixes; derivational morphemes definitely included, but were hardly limited to, so called formatives (also known as transitivity prefixes, cf. Oliveira 2005, Salanova 2011b, Nikulin \& Salanova forthc.). These topics should be covered in future research.

\section{Data provenance}

\begin{tabular}{|c|c|}
\hline Panará & Bardagil-Mas 2018, Dourado 2001, Lapierre ms., Vasconcelos 2013 \\
\hline Southern Kayapó & Barbosa 1918 \\
\hline Xavánte & Estevam 2011, Hall et al. 1987, Lachnitt 1987 \\
\hline Xerénte & $\begin{array}{l}\text { Krieger \& Krieger 1994, Sousa Filho 2007, Souza 2008, Eneida Brupahi Xerente p. c., } \\
\text { Mário André Coelho da Silva p. c. }\end{array}$ \\
\hline Kaingáng & Wiesemann 2002 \\
\hline Laklãnõ (= Xokléng) & Alves Jr 2014, Bublitz 1994, Gakran 2015, Jolkesky \& Gakran ms. \\
\hline Ingain & $\begin{array}{l}\text { Ambrosetti } 1896 \text { (Pedro, Cosme Román, María Antonia), Lista } 1883 \text { (Lista), Vogt } 1904 \\
\quad\left(\text { Vogt }^{1}, \text { Vogt }^{3}\right)\end{array}$ \\
\hline
\end{tabular}

The PNJ reconstructions are based on data from numerous sources specified in Nikulin \& Salanova (forthc.). For sources on Macro-Jê languages other than Jê, the reader is referred to Nikulin \& Silva (forthc.).

\section{References}

Aikio, Ante (Luobbal Sámmol Sámmol Ánte). 2012. On Finnic long vowels, Samoyed vowel sequences, and ProtoUralic * $x$. In: Tiina Hyytiäinen, Tiina Jalava, Janne Saarikivi, Erika Sandman (eds.). Per Urales ad Orientem. Iter polyphonicum multilingue. Festskrift tillägnad Juha Janhunen på hans sextioårsdag den 12 februari 2012: 227-250. Helsinki: Suomalais-Ugrilainen Seura. (Suomalais-Ugrilaisen Seuran Toimituksia, 264.) 
Aikio, Ante (Luobbal Sámmol Sámmol Ánte). 2015. The Finnic 'secondary e-stems' and Proto-Uralic vocalism. Suomalais-Ugrilaisen Seuran Aikakauskirja 95: 25-66.

Alves Jr, Ozias. 2014. Parlons xokleng / laklãnõ. Langue indigène du sud du Brésil. Paris: L'Harmattan.

Ambrosetti, Juan Bautista. 1896. Materiales para el estudio de las lenguas del grupo Kaingangue (Alto Paraná). Boletín de la Academia Nacional de Ciencias de Córdoba 14: 331-380.

Barbosa, Alexandre de Souza. 1918. Cayapó e panará. Uberaba: manuscript. Available online at: <http://etnolinguistica.wdfiles.com/local--files/biblio\%3Abarbosa-1918-cayapo/barbosa_1918_cayapo.pdf>

Bardagil-Mas, Bernat. 2018. Case and agreement in Panará (Naamval en congruentie in het Panará). PhD. Groningen: Rijksuniversiteit Groningen.

Benware, Wilbur A. 1974 [1995]. The Study of Indo-European Vocalism in the 19th century: From the beginnings to Whitney and Scherer. Amsterdam, Philadelphia: John Benjamins. (Studies in the History of the Language Sciences, 3.)

Bishop, Nancy. 1996. A preliminary description of Kensiw (Maniq) phonology. Mon-Khmer Studies 25: $227-253$.

Bublitz, Terezinha. 1994. Análise fonológica preliminar da língua Xokléng. MA thesis. Brasília: Universidade de Brasília.

Carvalho, Fernando O. de. 2016. On the Development of the Proto-Northern Jê Rhotic in Panará Historical Phonology. Anthropological Linguistics 58(1): 52-78.

Carvalho, Fernando O. de, Andrey Nikulin. In preparation. Internal classification of Tupian.

Castro Alves, Flávia de. 2010. Evolution of Alignment in Timbira. International Journal of American Linguistics 76(4): 439-475.

Constenla Umaña, Adolfo. 2000. La restitución: un método lingüístico reconstructivo sincrónico. Filología y Lingüística 26(2): 161-180.

D’Angelis, Wilmar da Rocha. 2002. Gênero em Kaingang? In: Ludoviko dos Santos, Ismael Pontes (orgs.). Línguas Jê: estudos vários: 215-242. Londrina: Eduel.

Davis, Irvine. 1966. Comparative Jê phonology. Estudos Lingüísticos: Revista Brasileira de Lingüística Teórica e Aplicada 1(2): 20-24.

Dourado, Luciana Gonçalves. 2001. Aspectos morfossintáticos da língua Panará (Jê). PhD. Campinas: Universidade Estadual de Campinas.

Estevam, Adriana Machado. 2011. Morphosyntaxe du xavante. Langue jê du Mato Grosso (Brésil). PhD. Paris: Université Paris Diderot (Paris 7).

Gakran, Nanblá. 2015. Elementos fundamentais da gramática Laklãnõ. PhD. Brasília: Universidade de Brasília.

Hall, Joan, Ruth Alice McLeod, Valerie Mitchell. 1987 [2004]. Pequeno dicionário Xavante-Português, PortuguêsXavante. Damreme pibuzé hã a'uwẽ mreme, warazu mreme na te te ĩsaprĩ mono zém na duré warazu mreme, a'uwẽ mreme na te te ĩsaprĩ mono zém na. Cuiabá: Sociedade Internacional de Lingüística.

Jolkesky, Marcelo Pinho de Valhery. 2010. Reconstrução fonológica e lexical do Proto-Jê Meridional. MA thesis. Campinas: Universidade Estadual de Campinas.

Jolkesky, Marcelo Pinho de Valhery, Nanblá Gakran. Ms. As classes verbais em Xokleng. Available online at: $<$ https://www.academia.edu/5278237/As_classes_verbais_em_xokleng>.

Kassian, Alexei. 2015. Towards a formal genealogical classification of the Lezgian languages (North Caucasus): testing various phylogenetic methods on lexical data. PLoS ONE 10(2): e0116950.

Krieger, Wanda Braidotti, Guenther Carlos Krieger (orgs.). 1994. Dicionário escolar: Xerente-Português; PortuguêsXerente. Rio de Janeiro: Junta das Missões Nacionais da Convenção Batista Brasileira.

Lachnitt, Georg. 1987. Romnhitsi'ubumro. A'uwẽ mreme = waradzu mreme. Dicionário xavante = português. Campo Grande: Missão Salesiana de Mato Grosso.

Ladefoged, Peter, Ian Maddieson. 1990. Vowels of the world's languages. Journal of Phonetics 18: $93-122$.

Lapierre, Myriam. Ms. A phonological analysis of Panãra. Berkeley: University of California.

Lista, Ramón. 1883. El Territorio de Las Misiones. Buenos Aires: Imprenta «La Universidad», de J. N. Klingelfuss.

Nikulin, Andrey. 2015. Apofonia e sistema vocálico do Proto-Jê Meridional: contribuição para estudos comparativos das línguas Jê. Revista Brasileira de Linguística Antropológica 7(2): 275-306.

Nikulin, Andrey. 2016. Historical phonology of Proto-Northern Jê. Journal of Language Relationship 14(3): 165-185.

Nikulin, Andrey. 2017. A phonological reconstruction of Proto-Cerrado (Jê family). Journal of Language Relationship 15(3): 147-180.

Nikulin, Andrey, Andrés Pablo Salanova. Forthc. Northern Jê verb morphology and the reconstruction of finiteness alternations. International Journal of American Linguistics 85(4). 
Nikulin, Andrey, Mário André Coelho da Silva. Forthc. As línguas Maxakalí e Krenák dentro do tronco Macro-Jê. Cadernos de Etnolingüística (Estudos de Lingüística Sul-Americana) 7.

Nikulin, Andrey, Mário André Coelho da Silva. Forthc. A evolução do alinhamento na língua Maxakalí. In: Christiane Cunha de Oliveira (org.). Memórias do II Encontro dos Americanistas no Cerrado. Goiânia: Universidade Federal de Goiás.

Oliveira, Christiane Cunha de. 2005. The language of the Apinajé people of Central Brazil. PhD. Eugene: University of Oregon.

Pache, Matthias. 2018. Contributions to Chibchan Historical Linguistics. PhD. Leiden: Universiteit Leiden.

Ribeiro, Eduardo Rivail. 2004. Prefixos relacionais em Jê e Karajá: um estudo histórico-comparativo. Línguas Indígenas Americanas 4: 91-101.

Ribeiro, Eduardo Rivail. 2011. Prefixos relacionais como evidência histórico-comparativa: os casos Chiquitano e Jabutí. In: Ana Suelly Arruda Câmara Cabral, Aryon Dall'Igna Rodrigues, Jorge Domingues Lopes, Maria Risolêta Silva Julião (orgs.). Línguas e Culturas Macro-Jê, vol. 2: 105-120. Campinas: Curt Nimuendajú; Brasília: Laboratório de Línguas Indígenas.

Ribeiro, Eduardo Rivail. 2012. A grammar of Karajá. PhD. Chicago: University of Chicago.

Ribeiro, Eduardo Rivail, Hein van der Voort. 2010. Nimuendajú was right: The inclusion of the Jabutí language family in the Macro-Jê stock. International Journal of American Linguistics 76(4): 517-570.

Salanova, Andrés Pablo. 2011a. A flexão de terceira pessoa nas línguas Jê. Línguas Indígenas Americanas 11: 75-114.

Salanova, Andrés Pablo. 2011b. Reduplication and number in Mẽbengokre. In: Gale Goodwin Gómez, Hein van der Voort (eds.). Reduplication in Indigenous Languages of South America: 252-273. Leiden: Brill.

Sousa Filho, Sinval Martins. 2007. Aspectos morfossintáticos da língua Akwẽe-Xerente (Jê). PhD. Goiânia: Universidade Federal de Goiás.

Souza, Shelton Lima de. 2008. Descrição fonético-fonológica da língua akwen-xerente. MA thesis. Brasília: Universidade de Brasília.

Traunmüller, Hartmut. 1982. Der Vokalismus im Ostmittelbairischen. Zeitschrift für Dia-lektologie und Linguistik 49: 289-333.

Vasconcelos, Eduardo Alves. 2013. Investigando a hipótese Cayapó do Sul-Panará. PhD. Campinas: Universidade Estadual de Campinas.

Vogt, Federico. 1904. Die Indianer des Obern Paraná: II. Die Indianer der Coroados-Gruppe. Mitteilungen der Anthropologischen Gessellschaft in Wien 34(4): 353-377.

Wiesemann, Ursula. 1978. Os dialetos da língua Kaingáng e o Xokléng. Arquivos de Anatomia e Antropologia 3: $199-217$. Wiesemann, Ursula Gojtéj. 2002. Dicionário Kaingang-Português, Português-Kaingang. Curitiba: Esperança.

\section{А. В. Никулин. Реконструкция фонологии и словарного состава пра-же языка}

В статье рассматриваются регулярные фонетические соответствия между праязыками нагорной (Никулин 2017) и южной (Жолкески 2010) ветвями языковой группы же, самой глубокой генетической единицы в составе южноамериканской семьи макро-же. На основании этих соответствий предлагается реконструкция фонологии праязыка семьи же. Автор восстанавливает 11 согласных и 19 гласных фонем. Максимальная структура слога, восстанавливаемая для пра-же, $-{ }^{*} / \mathrm{CrVC} /$; при этом имеется ряд ограничений на сложные инициали (восстанавливаются только */pr, mr, kr, pr/). Автор восстанавливает правило, согласно которому носовые инициали имели в пра-же посторализованные аллофоны перед неносовыми централями. Статья завершается списком этимологий же.

Ключевые слова: языки же, языки макро-же, языковая реконструкция, сравнительноисторический метод. 